\title{
THE EFFECT OF A TRIPHASIC PULSE ON SCS TO ICD CROSSTALK
}

\author{
A Thesis \\ presented to \\ the Faculty of California Polytechnic State University, \\ San Luis Obispo
}

\author{
In Partial Fulfillment \\ of the Requirements for the Degree \\ Master of Science in Biomedical Engineering \\ by \\ Ryan James Wensley \\ June 2013
}


(C) 2013

Ryan James Wensley ALL RIGHTS RESERVED 


\section{COMMITTEE MEMBERSHIP}

TITLE:

AUTHOR:

DATE SUBMITTED:

COMMITTEE CHAIR:

COMMITTEE MEMBER: Lanny Griffin, Ph.D, Department Chair, Cal Poly, SLO

COMMITTEE MEMBER: Gene Bornzin, Ph.D, VP of Research, St. Jude Medical
The Effect of a Triphasic Pulse on SCS to ICD Crosstalk

Ryan James Wensley

June 2013 


\author{
ABSTRACT \\ The Effect of a Triphasic Pulse on SCS to ICD Crosstalk \\ Ryan James Wensley
}

It is a known problem that a Spinal Cord Stimulator (SCS) can interact with an Implantable Cardioverter Defibrillator (ICD) when both devices are implanted in the same patient. Interactions between the SCS and ICD can cause inappropriate therapy which can be harmful to the patient. While ICD devices have a distinct narrowband sensing bandwidth, the pulse configurations that current SCS devices deliver were not designed with this frequency region in mind. In this thesis, I recommend a new pulse configuration for SCS devices that will minimize the interaction between the two devices. I produce a theoretical equation for each pulse configuration in the frequency domain using the Laplace transform and present the results in Matlab. I also design my own SCS device to deliver multiple pulse configurations and use it to gather empirical data. The theoretical and empirical results are used to show the extent of the improvement between the new pulse and existing pulse configurations. The results prove that the new pulse configuration will significantly reduce crosstalk within the desired ICD bandwidth. A reduction in crosstalk will decrease the probability that an SCS will interact with a ICD device.

Keywords: ICD - Implantable Cardioverter Defibrillator, SCS - Spinal Cord Stimulator, VT Ventricular Tachycardia, VF - Ventricular Fibrillation, AF - Atrial Fibrillation, ECG Electrocardiogram, ATP - Antitachycardia Pacing, GUI - Graphical User Interface, $\mu \mathrm{C}-$ Microcontroller, USB - Universal Serial Bus, UART - Universal Asynchronous Receiver Transmitter, ACK - Acknowledged, NAK - Not Acknowledged, BPM - Beats Per Minute, R Resistance, C - Capacitance 


\section{ACKNOWLEDGMENTS}

I would like to thank St. Jude Medical for providing me the tools to complete this project. A special thanks to St. Jude Medical employees Gene Bornzin, Martin Cholette, and John Poore and my Dad, Roy Wensley, Ph.D, for all their help and knowledge on this subject matter. 


\section{TABLE OF CONTENTS}

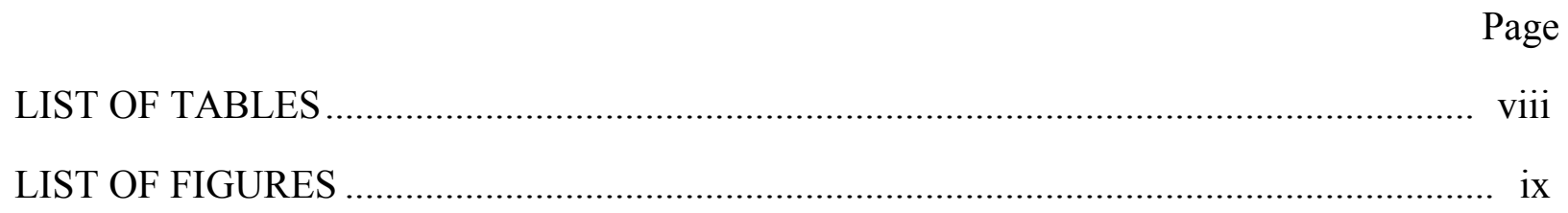
CHAPTER

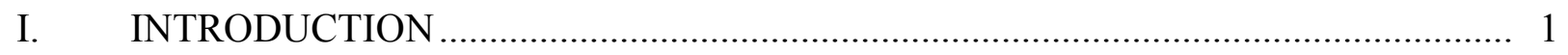

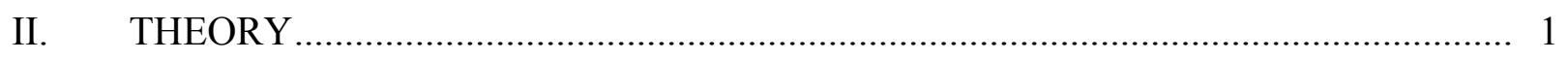

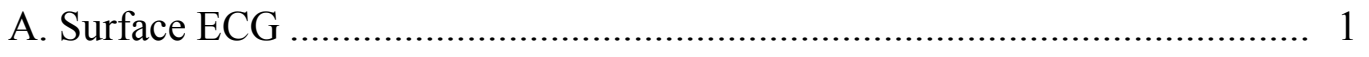

B. Implantable Cardioverter Defibrillator (ICD) Background ...................... 2

C. Spinal Cord Stimulator (SCS) Background ....................................... 7

D. Electrical Cross-Talk ................................................................... 9

E. Pulse Generator Design ............................................................. 14

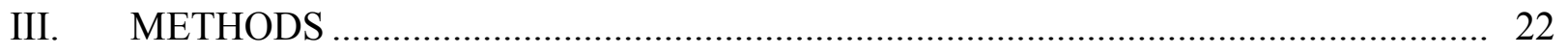

A. Analytical Experimental Method ................................................... 23

B. Saline Experimental Method .............................................................. 25

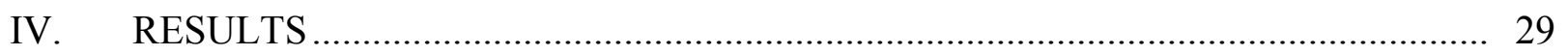

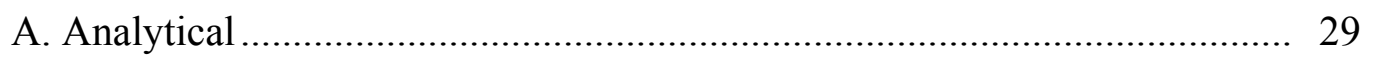

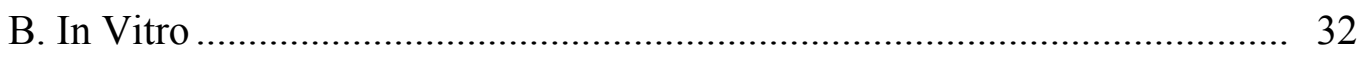

V. DISCUSSIONS AND CONCLUSIONS ....................................................... 34

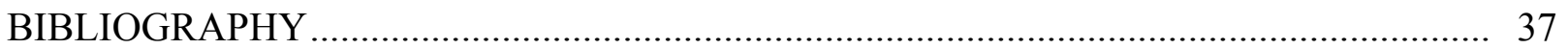




\section{APPENDICES}

A. Pulse Generator Waveforms ………………........................................... 39

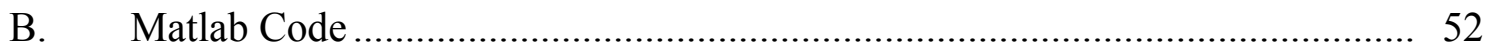

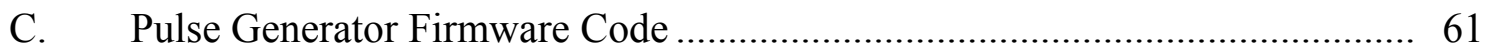

D. GUI Executable Code ………………………..................................... 91

E. Hardware Schematics ............................................................................. 103

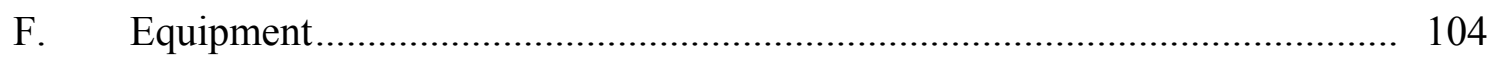




\section{LIST OF TABLES}

Table Page

1. UART Command and Parameter Code Descriptions ............................. 20

2. Pulse Period (Measured vs. Requested) ................................................ 32

3. $\quad \pm$ Pulse Width (Measured vs. Requested) ............................................ 33

4. Positive DAC voltage (Measured vs. Requested).................................. 33

5. Negative DAC voltage (Measured vs. Requested) ............................... 33

6. Maximum recorded negative and positive amplitudes for all 4 waveforms at $200 \mu \mathrm{s}$ and $500 \mu \mathrm{s}$ pulse widths................................................... 34

7. Equipment List for Saline Experiment ........................................... 104 


\section{LIST OF FIGURES}

Figure $\quad$ Page

1. Important deflections and intervals of a typical scalar ECG ....................... 2

2. In this demonstration of a single-lead ICD, the solid arrows demonstrate a shock with electricity flowing between the large proximal band and the wall of the can, which represents the other pole. The generator is usually placed in the left anterior chest, which allows more of the electricity to go through the left ventricle in an effort to defibrillate it. The tip and band electrode of the bipolar pacemaker are shown with a separate electric current available for

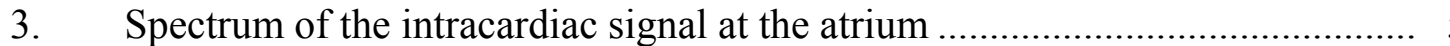

4. The two methods of antitachycardia pacing. A: Burst pacing is illustrated using the ramp sequence. Note the "within burst" decrement of cycle length. B: In the scan sequence or "between burst" decrement, the cycle length is constant during any given burst, but it decreases progressively with each successive train.

5. Typical biphasic shock waveform. T1 is the beginning of the positive pulse segment; T2 is the end of the positive pulse; T3 and T4 are the beginning and end, respectively, of the negative pulse segment. The leading-edge voltage VL is the peak value of the voltage.

6. X-Ray of an SCS Lead and Device Implanted in a Patient ........................... 8

7. The Output Signal of Device \#1 (50Hz) Compared to the Sensed Signal of Device \#2 after 21-105Hz Bandpass Filter. Note: The filter used was a 2nd order bandpass

8. The Output Signal of Device \#1 $(500 \mathrm{~Hz})$ Compared to the Sensed Signal of Device \#2 after 21-105Hz Bandpass Filter. Note: The filter used was a 2nd order bandpass

9. Magnitude response plot of a monophasic pulse with a pulse width, $\mathrm{T}=50 \mu \mathrm{s}$

10. The electric dipole. 13

11. Pulse Generator Diagram. 
Figure

12. Microcontroller Firmware State Diagram................................................ 17

13. Pulse Generator GUI Executable ........................................................... 22

14. General flow outline of the saline experiment and data post-processing ....... 28

15. Magnitude response at $\mathrm{T}=50 \mu \mathrm{s}$ with no interphasic gap ........................ 29

16. Magnitude response at $\mathrm{T}=200 \mu \mathrm{s}$ with no interphasic gap ....................... 30

17. Magnitude response at $\mathrm{T}=500 \mu \mathrm{s}$ with no interphasic gap ...................... 30

18. Magnitude response at $\mathrm{T}=50 \mu \mathrm{s}$ with no interphasic gap (zoomed out)........ 31

19. Magnitude response for a Triphasic waveform at $\mathrm{T}=50 \mu \mathrm{s}$ with an

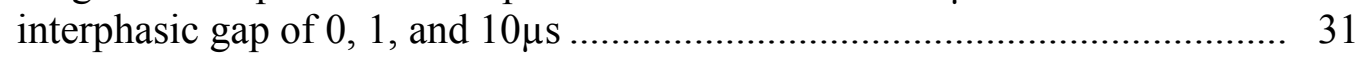

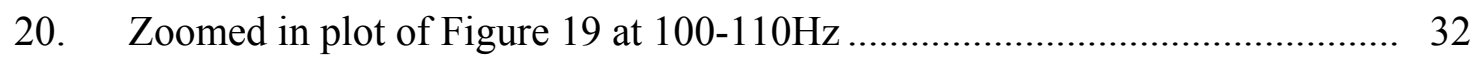

21. Ideal monophasic waveform with $50 \mu$ s pulse width ................................. 39

22. Ideal biphasic waveform with $50 \mu$ s pulse width ................................... 39

23. Ideal triphasic waveform with $50 \mu \mathrm{s}$ pulse width ...................................... 40

24. Ideal pentapolar waveform with $50 \mu$ s pulse width .................................. 40

25. Ideal triphasic waveform with $50 \mu$ s pulse width and a $1 \mu$ s interphasic delay....

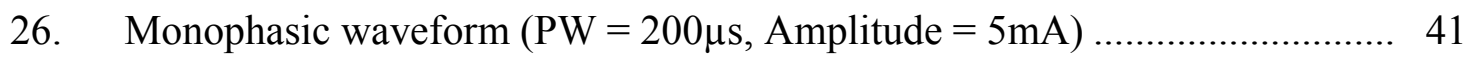

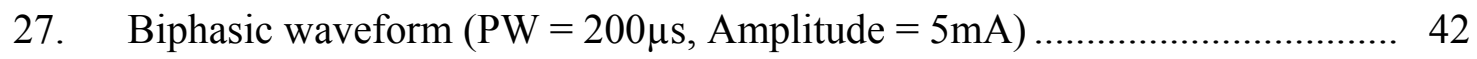

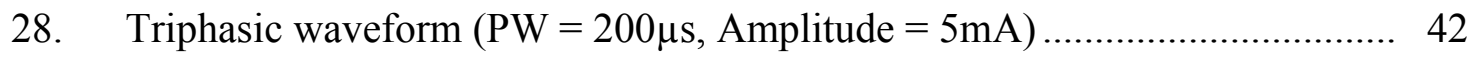

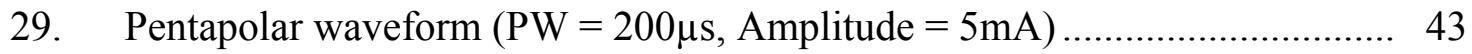

30. Monophasic ICD and SCS waveforms before the sense filter is applied $(\mathrm{PW}=200 \mu \mathrm{s})$. 
31. Biphasic ICD and SCS waveforms before the sense filter is applied $(\mathrm{PW}=200 \mu \mathrm{s})$.

32. Triphasic ICD and SCS waveforms before the sense filter is applied $(\mathrm{PW}=200 \mu \mathrm{s})$.

33. Pentapolar ICD and SCS waveforms before the sense filter is applied $(\mathrm{PW}=200 \mu \mathrm{s})$.

34. All 4 ICD waveforms post sense filter with a programmed pulse width $=200 \mu \mathrm{s}$ 46

35. Biphasic, Triphasic, and Pentapolar ICD waveforms post sense filter with a programmed pulse width $=200 \mu \mathrm{s}$.

36. Monophasic ICD and SCS waveforms before the sense filter is applied $(\mathrm{PW}=500 \mu \mathrm{s})$.

37. Biphasic ICD and SCS waveforms before the sense filter is applied $(\mathrm{PW}=500 \mu \mathrm{s})$.

38. Triphasic ICD and SCS waveforms before the sense filter is applied $(\mathrm{PW}=500 \mu \mathrm{s})$...

39. Pentapolar ICD and SCS waveforms before the sense filter is applied $(\mathrm{PW}=500 \mu \mathrm{s})$.

40. All 4 ICD waveforms post sense filter with a programmed pulse width $=500 \mu \mathrm{s}$

41. Biphasic, Triphasic, and Pentapolar ICD waveforms post sense filter with

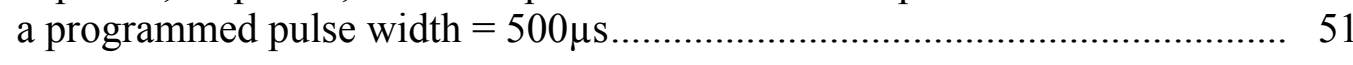

42. V-I Pulse Converter ........................................................................ 103 


\section{Introduction}

Implantable Cardiac Defibrillators (ICD) and cardiac pacemakers are designed to sense (detect) electrical signals inside the heart and inhibit or provide therapy depending on the amplitude and shape of that electrical signal (Moses, Miller, Moulton, \& Schneider, 2000, pp. 55-56). An inherent risk of providing therapy based on sensing, is the possibility of sensing other electrical signals and misinterpreting them (Andersen, Oxhoj, \& Arnsbo, 1990). These electrical signals can originate in the body (e.g. action potentials from skeletal muscle) or from external sources (e.g. electrocautery). This is known as cross-talk or over-sensing (Tidy \& Rull, 2010).

Spinal Cord Stimulators (SCS), unlike ICDs, do not sense; they only emit electrical signals into the spinal cord tissue (Andersen, Oxhoj, \& Arnsbo, 1990). SCS devices have been developed over the past 15+ years (Molon, et al., 2011) and today are a well-established treatment for chronic pain (Andersen, Oxhoj, \& Arnsbo, 1990). There are also many new areas that SCS therapy is being looked into, such as patients with ventricular arrhythmias (Vaseghi \& Shivkumar, 2012) and atrial fibrillation (Bernstein, et al., 2012), that also traditionally involve pacemaker or ICD therapy. This possible overlap in therapy, gives reason to believe that the amount of cases where both an ICD and SCS device being implanted in the same patient may increase.

Since the ICD will sense electrical signals and the SCS will emit electrical signals, there is potential risk of cross-talk when both devices are implanted in the same patient (Andersen, Oxhoj, \& Arnsbo, 1990). The research described in this document characterizes the potential cross-talk between the SCS and ICD devices with the current pulse configurations, monophasic and biphasic, and gives evidence that the amount of cross-talk can be significantly diminished by using a triphasic SCS pulse configuration.

\section{Theory}

\section{A. Surface ECG}

The heart contraction or heart beat causes a distinct electrical waveform known as a scalar electrocardiogram (ECG) (Koeppen \& Stanton, 2008). 


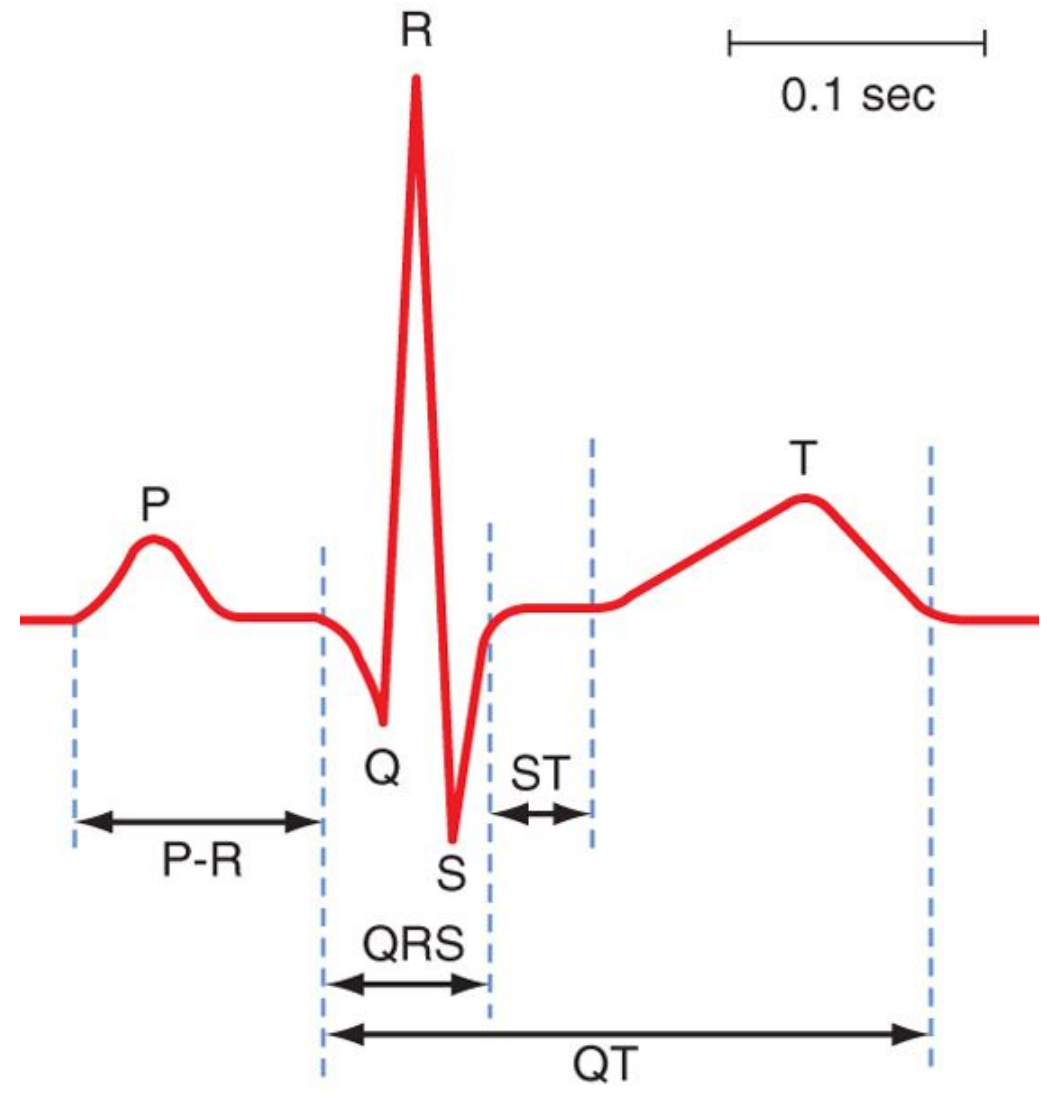

Figure 1: Important deflections and intervals of a typical scalar ECG (Koeppen \& Stanton, 2008, p. 311).

A typical scalar ECG is shown in Figure 1. The scalar ECG has 5 distinct portions or waves: P, $\mathrm{Q}, \mathrm{R}, \mathrm{S}$, and $\mathrm{T}$-waves. The $\mathrm{P}$-wave is produced by the depolarization of the atria which signals the start of an atrial contraction. The Q, R, and S-waves, known as the QRS complex, are produced by the depolarization of the ventricles which signals the start of a ventricle contraction. The atria repolarize somewhere within the QRS complex, but the ventricles generate such a large voltage deflection that it is hidden in the scalar ECG. Lastly, the T-wave is produced by the repolarization of the ventricles.

\section{B. Implantable Cardioverter Defibrillator (ICD) Background}

Originally the ICD was developed only to defibrillate or shock patients who developed ventricular tachycardia (VT) or ventricular fibrillation (VF). The current ICD models now combine all the features of a pacemaker, pacing and sensing, along with antitachycardia pacing (ATP), low-energy cardioversion, and the ability to defibrillate (Moses, Miller, Moulton, \& Schneider, 2000, p. 111). Pacing and shocking the heart both use electrical energy to stimulate cardiac tissue; however, they serve two completely different purposes. Pacing pulses use a voltage pulse, typically $<5 \mathrm{~V}$, to stimulate a localized part of the heart tissue to tell that section of the heart to depolarize and contract. A shock uses a voltage pulse, which can be as high as $890 \mathrm{~V}$ 
or 40J (St. Jude Medical, 2012) to capture (depolarize and reset) all of the heart tissue. Capturing all of the heart tissue will tell it to "reset". Figure 2 shows an example of a single-lead ICD and the difference between the shock path, which captures all of the heart tissue, and the pacing path, which captures a very small portion of the heart using a tip to band (or ring) electrode configuration.

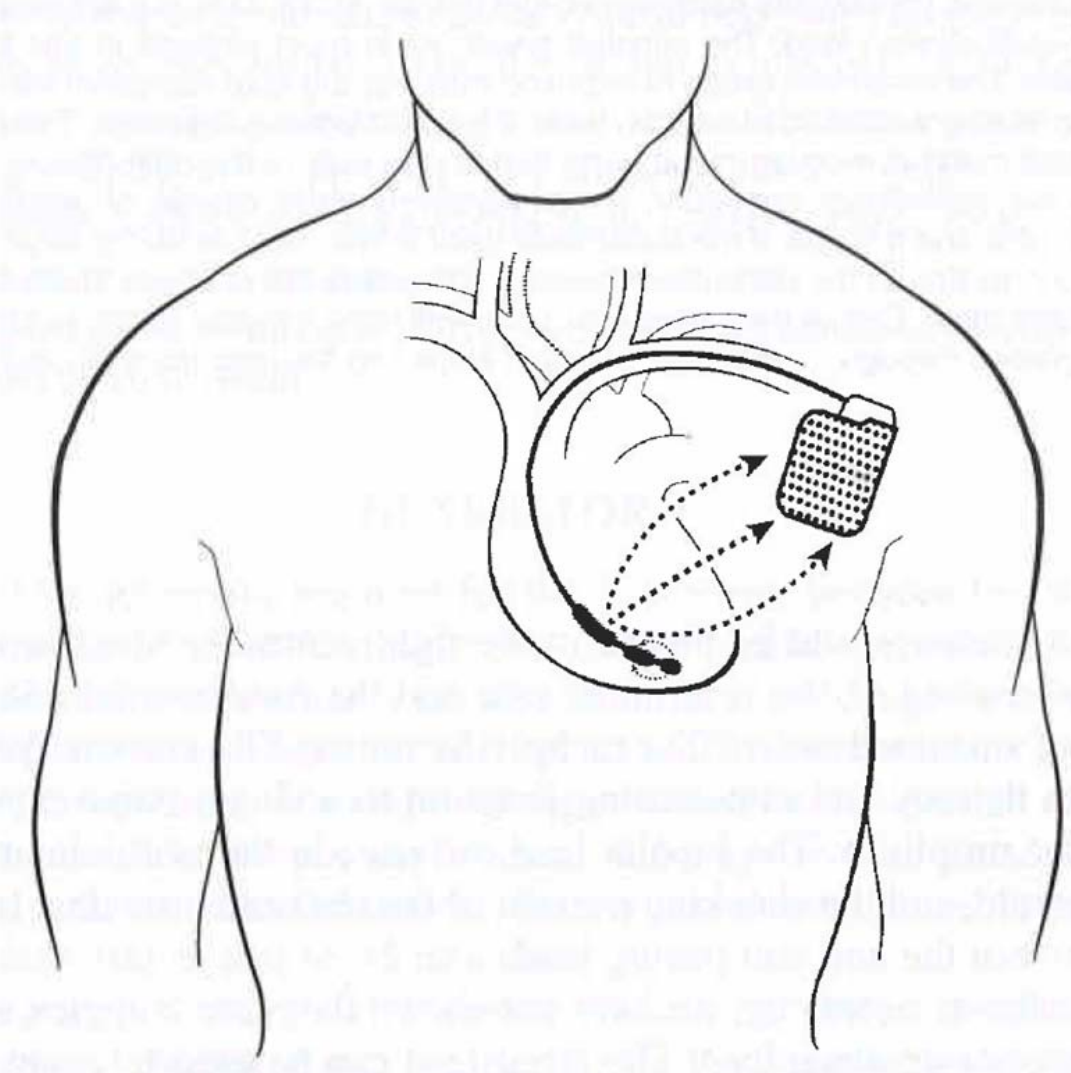

Figure 2: In this demonstration of a single-lead ICD, the solid arrows demonstrate a shock with electricity flowing between the large proximal band and the wall of the can, which represents the other pole. The generator is usually placed in the left anterior chest, which allows more of the electricity to go through the left ventricle in an effort to defibrillate it. The tip and band electrode of the bipolar pacemaker are shown with a separate electric current available for pacing (Moses, Miller, Moulton, \& Schneider, 2000, p. 114).

Another feature of the current ICD models is their ability to provide "Tiered therapy," which allows the doctor to programmatically set how the ICD will treat a patient when the device detects VT (Moses, Miller, Moulton, \& Schneider, 2000, p. 115). For example: The first tier of therapy could be set to provide ATP, if that is unsuccessful, the second tier could be a lowenergy cardioversion pulse, and then lastly, a high energy shock. High energy shocks have been observed to cause pain, so tiered therapy is a way to minimize the amount of pain towards the patient when treating VT (Moses, Miller, Moulton, \& Schneider, 2000, p. 120). Note: Defibrillation is the only therapy used when VF is detected. 


\section{Pacing and Sensing}

All modern pacemakers and ICDs are designed to sense the patient's intrinsic rhythm. The leads that go from the device to the patient (shown in Figure 2) have electrodes that can deliver therapy, i.e. pace or shock, or sense the electrical signals generated by the heart. The sense circuitry is designed to determine if a particular cardiac event occurred or not. For example: If a device is in VVI mode, its ventricular lead is looking (sensing) for a QRS complex (ventricular event). If no QRS complex is detected in a particular period of time, the device will pace the ventricle, stimulating a ventricular contraction (Moses, Miller, Moulton, \& Schneider, 2000, pp. 75-88). This type of sensing is also used to determine if the pacing rate is too fast, indicating that the patient is in VT. If a device is in AAI mode, its atrial lead is looking (sensing) for a P-wave. If no P-wave is detected in the specified amount of time, the device will pace the atrium stimulating an atrial contraction (Moses, Miller, Moulton, \& Schneider, 2000, pp. 75-88).

A cardiac event is sensed by setting an internal voltage threshold and comparing it to the voltage on a particular lead. To minimize the amount of cross-talk or over-sensing, the device utilizes blanking and refractory periods (Moses, Miller, Moulton, \& Schneider, 2000, pp. 168177) to stop monitoring the voltage for a specified amount of time after particular events have been sensed. For example: If the device paces the ventricle or senses a QRS complex, it will disable ventricle sensing for a particular amount of time because it knows that it is not possible for a QRS complex to occur again so quickly. This will reduce the risk of accidentally detecting VT due to cross-talk.

The ICD also has bandpass filters specifically designed to remove or block signals that do not share the frequency content of a cardiac event (see Electrical Cross-Talk section for more information). Figure 3 gives the frequency ranges for specific cardiac events as seen in the atrium. Since the leads are in the atrium, the ICD would be trying to sense for a P-wave. To successfully sense a P-wave and not another signal, the bandpass filter for this ICD channel would want to allow a frequency range around $80-100 \mathrm{~Hz}$. If the frequency range is too high, the ICD may accidentally sense muscle activity as a $\mathrm{P}$ wave. If the frequency range is too low, the ICD may accidentally sense a QRS complex or T wave as a P-wave. The specific bandwidth of these filters will vary slightly between the different ICD manufacturers but overall they will follow this general rule in their filter design. 


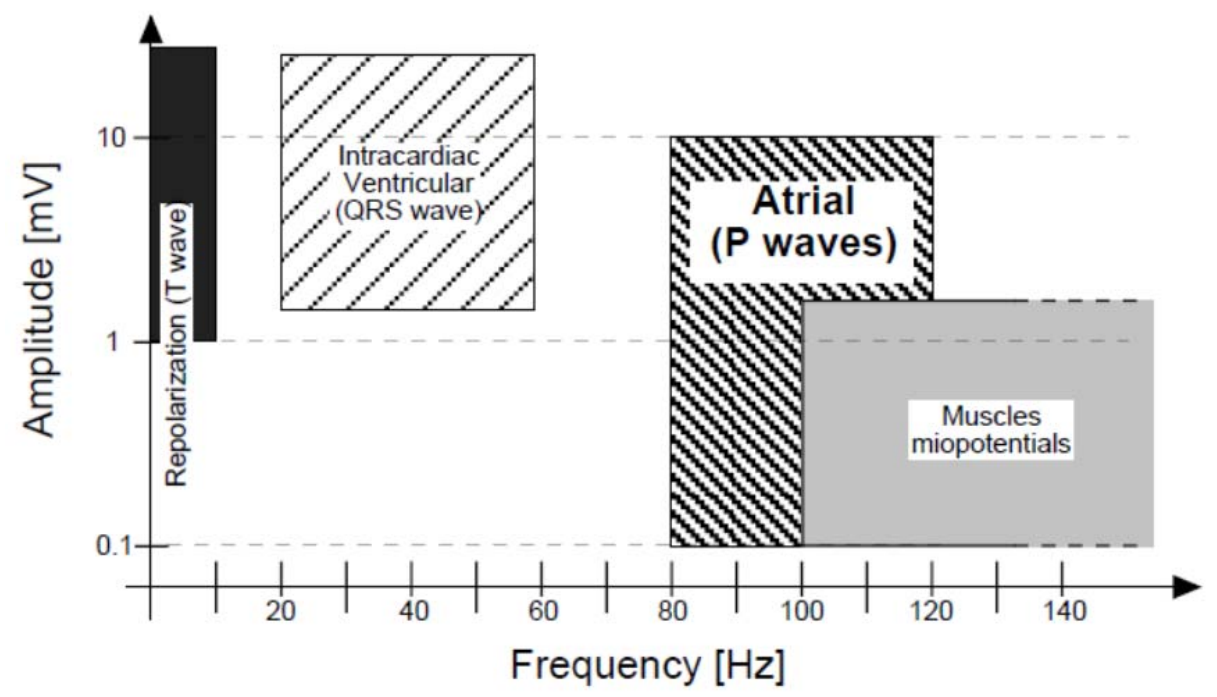

Figure 3: Spectrum of the intracardiac signal at the atrium (Mozzi, Neviani, \& Baschirotto, 2001). This is a general outline of the frequency content for some of the important intrinsic cardiac signals (P and QRS wave) and some of the unimportant electrical signals in the body ( $T$ wave and muscle miopotentials). It shows the importance on selecting the appropriate filter and bandwidth for the sense filter.

\section{Antitachycardia Pacing (ATP)}

Antitachycardia pacing, also known as over-drive pacing, is where the ICD sends a "burst" of pacing pulses at a fast rate in an effort to disrupt and eliminate the VT rhythm. There are two types of "burst" pacing:

1. Ramp pacing: Each pacing pulse within the burst is delivered at a faster rate (Figure 4A)

2. Scanning pacing: Each pacing burst is delivered at a faster rate (Figure 4B)

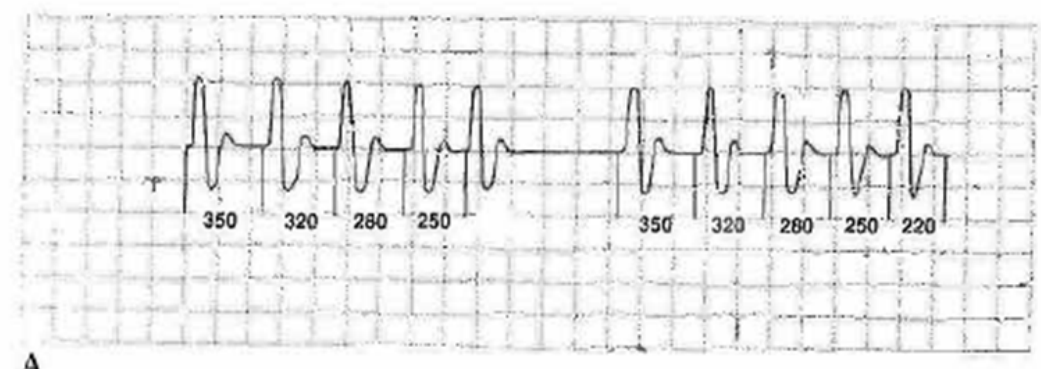

A

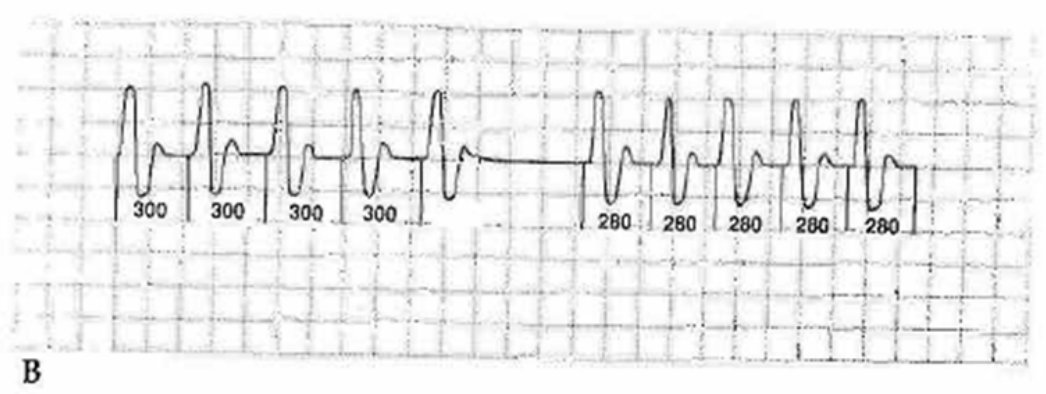


Figure 4: The two methods of antitachycardia pacing. A: Burst pacing is illustrated using the ramp sequence. Note the "within burst" decrement of cycle length. B: In the scan sequence or "between burst" decrement, the cycle length is constant during any given burst, but it decreases progressively with each successive train (Moses, Miller, Moulton, \& Schneider, 2000, p. 117).

This is usually the first-line therapy used with a VT rate $<180 \mathrm{bpm}$ (Moses, Miller, Moulton, \& Schneider, 2000). Using ATP provides a painless way to treat VT and requires less battery consumption. If ATP is unsuccessful, cardioversion or defibrillation is used as the next treatment therapy.

\section{Low-Energy Cardioversion}

Cardioversion is a low energy shock and can be programmed to deliver a shock with energy as low as $0.1 \mathrm{~J}$. This is significant because shocks at energies $>2 \mathrm{~J}$ have been observed to cause the patient pain (Moses, Miller, Moulton, \& Schneider, 2000). A cardioversion shock uses the exact same electronic circuitry that a high energy defibrillation uses, except it is designed to synchronize its delivery with the QRS complex. Recall: The QRS complex is the electrical indication that the ventricles are depolarizing and beginning to contract or pump. Synchronizing its delivery with the QRS complex avoids the T wave and reduces the risk of initiating VF. If ATP and/or cardioversion is unsuccessful in treating VT or VT evolves into VF, defibrillation therapy will be initiated (Moses, Miller, Moulton, \& Schneider, 2000).

\section{Defibrillation}

Defibrillation is the only therapy for when a patient presents with VF. Unlike low-energy cardioversion, the defibrillation shock does not need to synchronize with any cardiac event because the energy used in this therapy is enough to capture the entire heart. If the first shock does not terminate the VF rhythm, up to 5 subsequent shocks (6 in total) may be delivered (St. Jude Medical, 2012). The energy of these shocks can vary from $0.1 \mathrm{~J}$ to $40 \mathrm{~J}$. There has been a large amount of research focused on what type of waveform captures the heart the best. Figure 5 shows the current standard shock waveform: Biphasic. The energy of the shock can be calculated by the following equation: $E=\frac{1}{R} \int V(t)^{2}$ (Thammanomai, Sweeney, \& Eisenberg, 2006). 


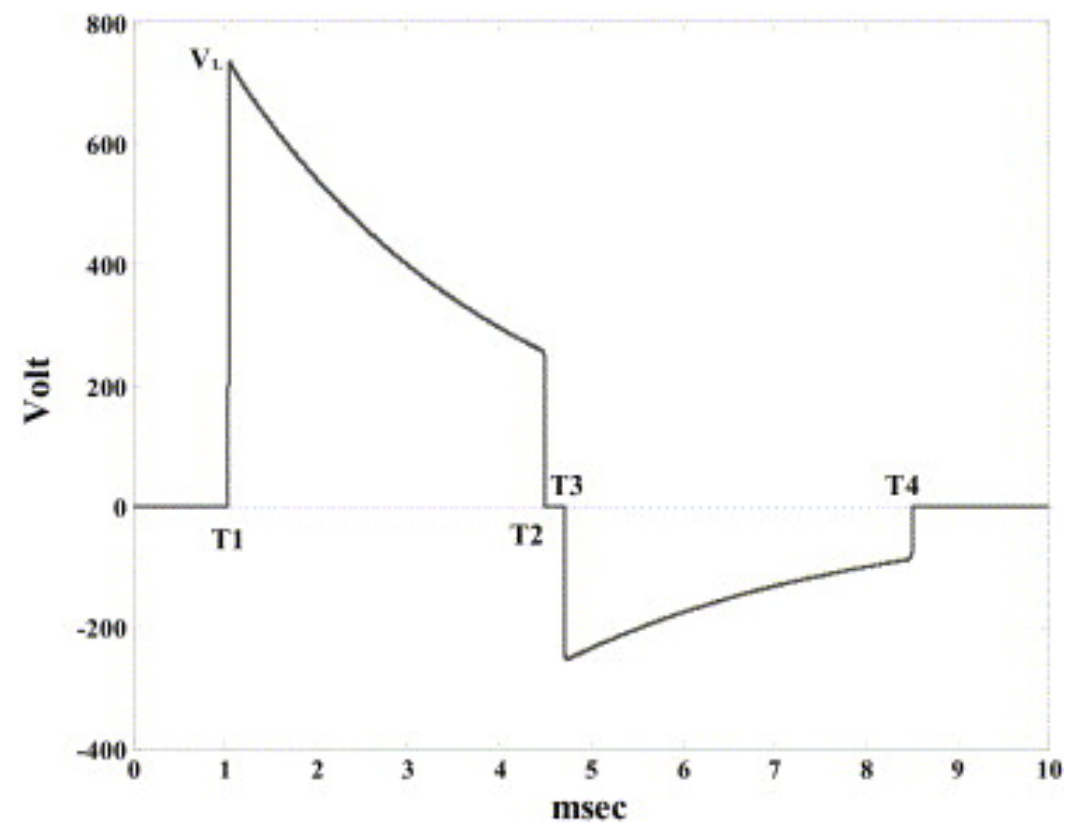

Figure 5: Typical biphasic shock waveform. T1 is the beginning of the positive pulse segment; T2 is the end of the positive pulse; T3 and T4 are the beginning and end, respectively, of the negative pulse segment. The leading-edge voltage VL is the peak value of the voltage (Thammanomai, Sweeney, \& Eisenberg, 2006).

The biphasic waveform delivers a positive pulse from T1 to T2, an interphasic gap from $\mathrm{T} 2$ to $\mathrm{T} 3$, and then a negative pulse from T3 to T4. The voltage decays with an RC constant that is dependent on the impedance of the patient, $\mathrm{R}$, and the capacitance, $\mathrm{C}$, of the device's internal capacitor.

\section{Spinal Cord Stimulator (SCS) Background}

Spinal cord stimulators (SCS) deliver electrical stimuli to segments of the spinal cord through implanted electrodes known as leads. SCS therapy typically treats chronic lower back pain and is most commonly indicated from failed back surgery syndrome (Stojanovic \& Abdi, 2002). The implant procedure is minimally invasive, which is leading more pain practitioners to use SCS therapy more often and earlier in the course of chronic low back pain (Stojanovic \& Abdi, 2002). Figure 6 shows an X-Ray of an SCS lead and device (Pulse Generator) implanted in a patient. The SCS leads are generally implanted in the thoracic epidural space with the tip of the lead somewhere within T8-T10 (Stojanovic \& Abdi, 2002). The other side of the lead is connected to an extension cable, and then to a pulse generator. The pulse generator will be implanted in the patient once the device and lead placement are in the correct place and approved by the physician based on a trial period. This trial period usually lasts from $24 \mathrm{hrs}-5$ days (Stojanovic \& Abdi, 2002). However, it can last as long as 28days according to Belgian health care requirements (Ridder, Plazier, Kamerling, Menovsky, \& Vanneste, 2013). 


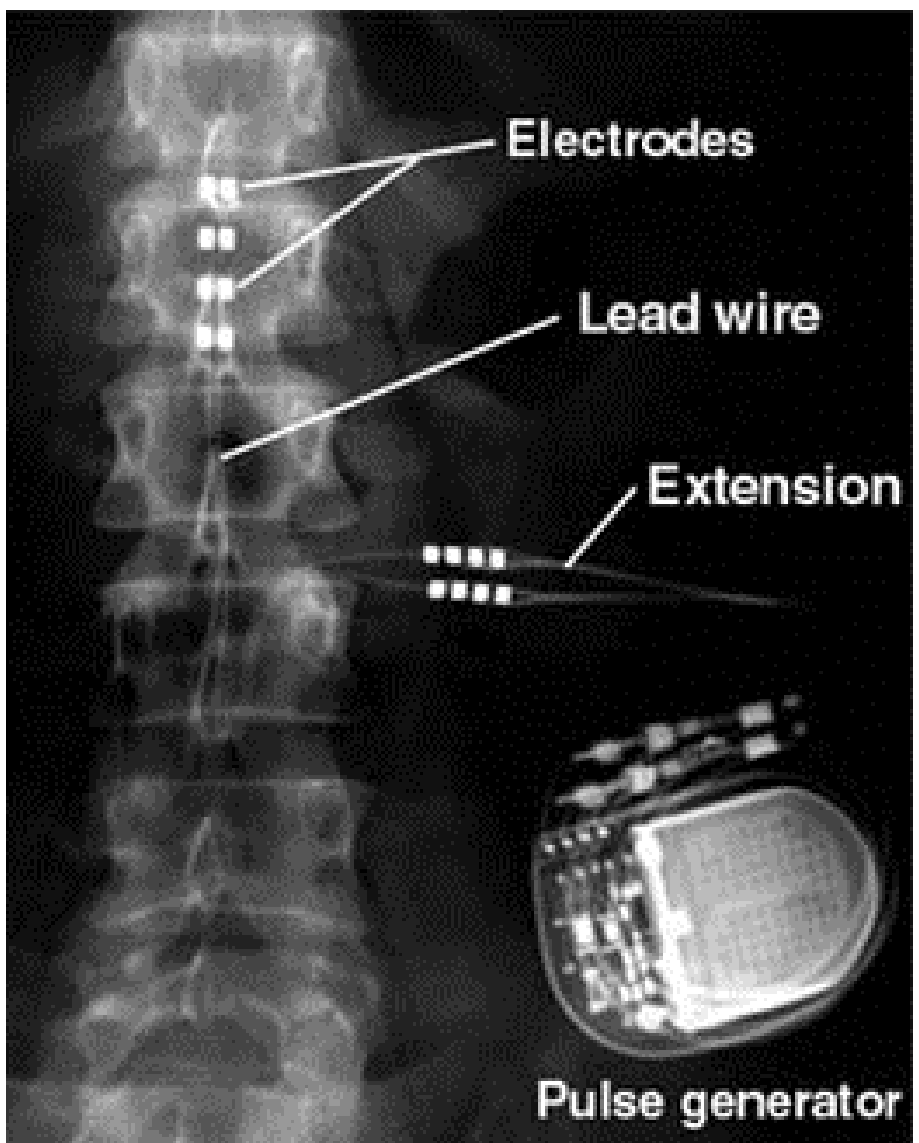

Figure 6: X-Ray of an SCS Lead and Device Implanted in a Patient (Dartmouth-Hitchcock, 2013)

During spinal cord stimulation therapy, the SCS device will produce an electrical field from the leads via a constant current or a constant voltage pulse. St. Jude Medical utilizes a constant current pulse. The two types of current pulse waveforms on the market today are monophasic and biphasic. The electric field generated by this pulse is designed to stimulate fibers inside the spine. SCS devices, such as St. Jude Medical's Eon Mini ${ }^{\mathrm{TM}}$, can adjust the pulse width, period, amplitude, and electrode configuration by an external programmer. This programmability allows the SCS to provide better stimulation coverage for the patient. The pulse generator design, described in the Pulse Generator Design section, based its specifications off of the Eon Mini ${ }^{\mathrm{TM}}$.

Electrical stimulation was first used clinically to control pain in 1967 (Shealy, Mortimer, \& Reswick, 1967) and was initially based on gate control theory by Melzack and Wall (Wall \& Melzack, 1965). This theory proposed that stimulating A-beta fibers reduces the nociceptive input from the periphery (Stojanovic \& Abdi, 2002). Basically, the SCS reduces or "gates" the patient's perception of pain by stimulating specific fibers in the spinal cord. Recently, more 
mechanisms have been discovered that may play a more significant role in the actions of SCS therapy (Stojanovic \& Abdi, 2002).

Along with new mechanisms being discovered, there are studies being performed indicating new areas that SCS therapy may be beneficial, which include indications for treating various cardiac diseases. Publications have linked SCS devices as possible therapies for the following:

- Angina (Taylor, Vries, Buchser, \& DeJongste, 2009)

- Myocardial ischemia (Dejongste, Terhorst, \& Foreman, 2009)

- Ventricular arrhythmias (Vaseghi \& Shivkumar, 2012)

- Atrial fibrillation (Bernstein, et al., 2012)

Along with the above publications, there are two large clinical trials that are currently underway:

- DEFEAT-HF by Medtronic (Medtronic CRMD, 2013)

- SCS-HEART by St. Jude Medical (St. Jude Medical, 2013)

These clinical trials aim to prove the efficacy of SCS therapy on patients with heart failure. With this large focus on SCS being a possible therapy for cardiac diseases, there is reason to believe that more patients may be indicated for an ICD (or pacemaker) and an SCS device in the future. This makes cohabitation between these two devices even more important.

\section{Electrical Cross-Talk}

Electrical cross-talk is a common problem in complex electronic systems. Multiple sources in the same system can interact with each other causing voltages to be seen in unintended places. ICD devices look or sense for a voltage to reach a certain threshold while SCS devices emit voltages. If an ICD senses cross-talk as an intrinsic cardiac event, it can cause unintended therapy such as pacing, ATP, cardioversion, or defibrillation. In an effort to get rid of the unwanted voltages, electrical devices will use filters to allow them to only see the frequency content that they desire. For example, if device \#1 (SCS) outputs a sinusoid at $50 \mathrm{~Hz}$ and device \#2 (ICD) uses a Bandpass filter to remove all frequencies outside of $21-105 \mathrm{~Hz}$, device \#2 will see the entire signal (Figure 7). However, if device $\# 1$ outputs a sinusoid outside of this frequency, $500 \mathrm{~Hz}$ for example, device $\# 2$ will see very little of that signal (Figure 8). 


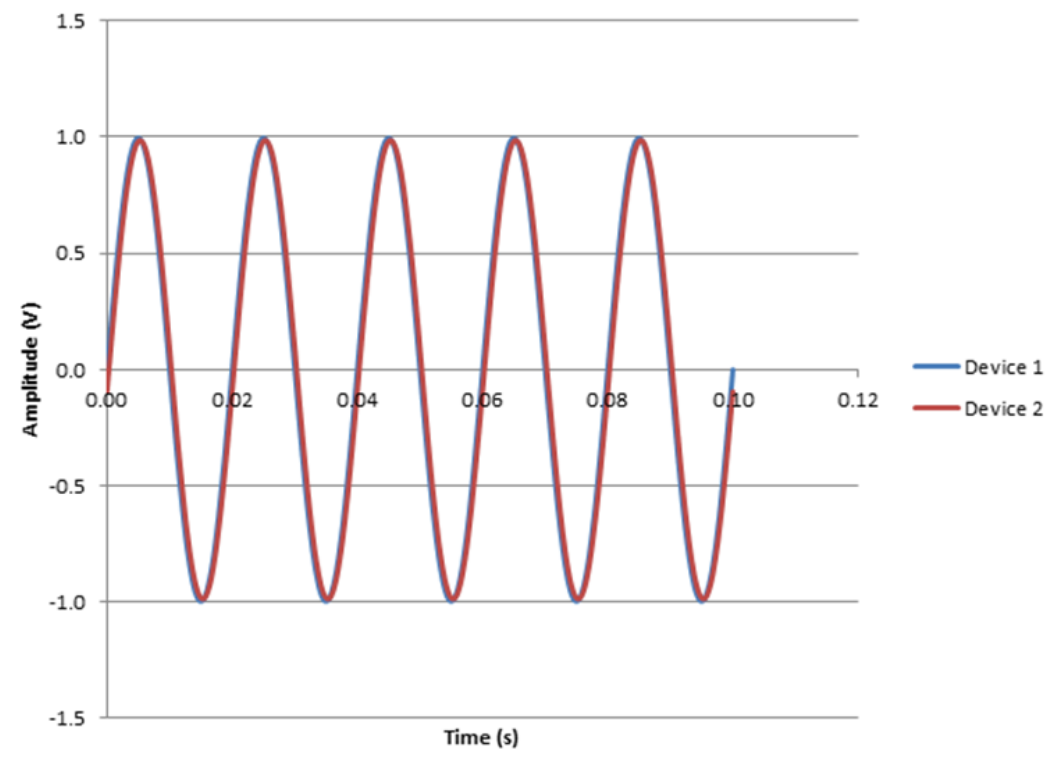

Figure 7: The Output Signal of Device \#1 $(50 \mathrm{~Hz})$ Compared to the Sensed Signal of Device \#2 after 21-105Hz Bandpass Filter. Note: The filter used was a $2^{\text {nd }}$ order bandpass.

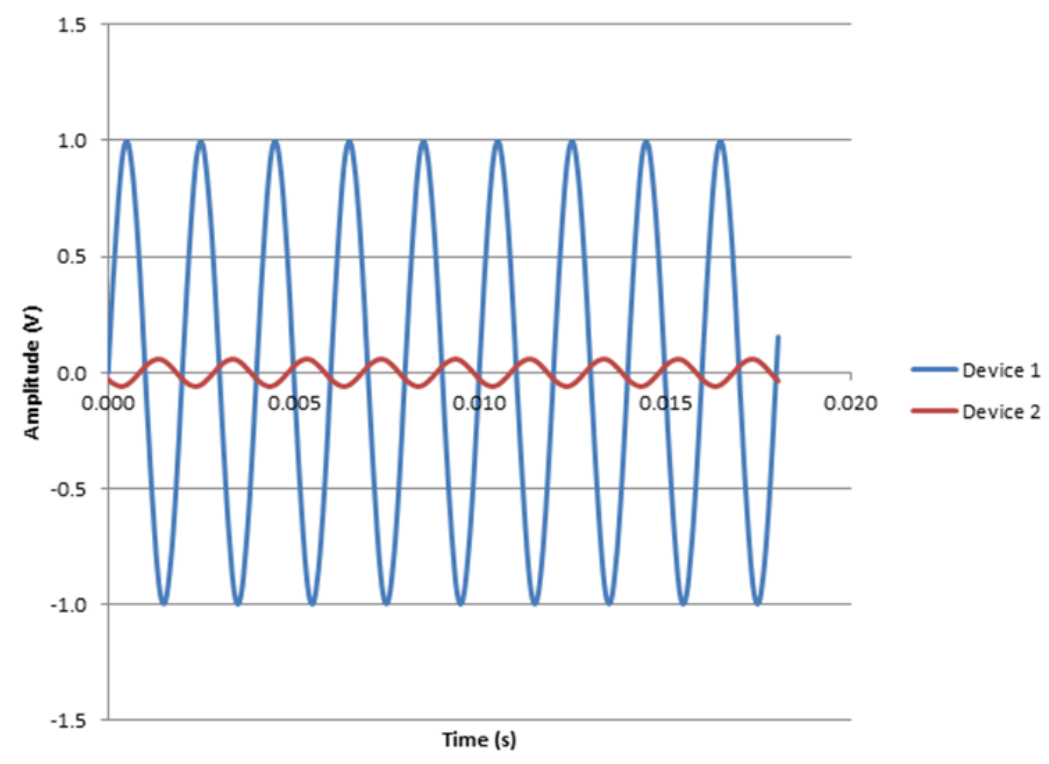

Figure 8: The Output Signal of Device \#1 (500Hz) Compared to the Sensed Signal of Device \#2 after 21-105Hz Bandpass Filter. Note: The filter used was a $2^{\text {nd }}$ order bandpass.

Another important item when dealing with cross-talk is the amplitude of the signal. The signal that a device is looking for can sometimes be very small in amplitude. For example: The signals that an ICD or pacemaker look for when monitoring the heart's intrinsic rhythm is on the order of millivolts (Moses, Miller, Moulton, \& Schneider, 2000). A large enough signal from another source, an SCS device for example, could be detected by the ICD even though the ICD 
has its own Bandpass filters. The size and shape of the SCS pulse will determine how much of the signal is seen by the ICD and if it is sense it or not.

Currently, ICDs (or pacemakers) and SCS device can be used in the same patient. However, there are precautions that the doctor must follow if this scenario occurs because of the possibility of cross-talk. For example: The SCS device frequency is limited to $20 \mathrm{~Hz}$ if implanted in a person with a pacemaker or ICD (Stojanovic \& Abdi, 2002). If the cross-talk between the two devices could be minimized by use of the triphasic pulse, the precautions may be able to be relaxed or eliminated.

\section{Laplace Transform}

The Laplace transform can be used to analytically convert a time-based signal into the frequency domain. Once in the frequency domain, the signal's frequency content can be determined to see how much energy it contains within the desired frequencies. The Laplace transform's general equation:

$$
F(s)=\int_{0}^{\infty} e^{-s t} f(t)
$$

Assuming a signal, " $\mathrm{f}(\mathrm{t})$ ", is linear and time-invariant (LTI), the general equation above can be simplified into many different properties. One property that is useful is the "delayed unit step" property:

$$
f(t)=u(t-\tau) \overrightarrow{\text { Laplace }} F(s)=\frac{e^{-\tau s}}{s}
$$

For example, a pulse that lasts for $\mathrm{T}$ seconds can be written in the time domain as the following:

$$
f(t)=-u\left(t-\frac{T}{2}\right)+u\left(t+\frac{T}{2}\right)
$$

This means that the function $=1$ from time, $-\mathrm{T} / 2$, to time, $\mathrm{T} / 2$, otherwise the function equals 0 . We can then use the "delayed unit step" property to rewrite this in the frequency domain:

$$
F(s)=\frac{e^{-\left(\frac{T}{2}\right) s}}{s}+\frac{e^{\left(\frac{T}{2}\right) s}}{s} \text { where } s=j 2 \pi f
$$

Now that we have the equation in the frequency domain, we can use Matlab to visually see the frequency content of the signal (Figure 9). 


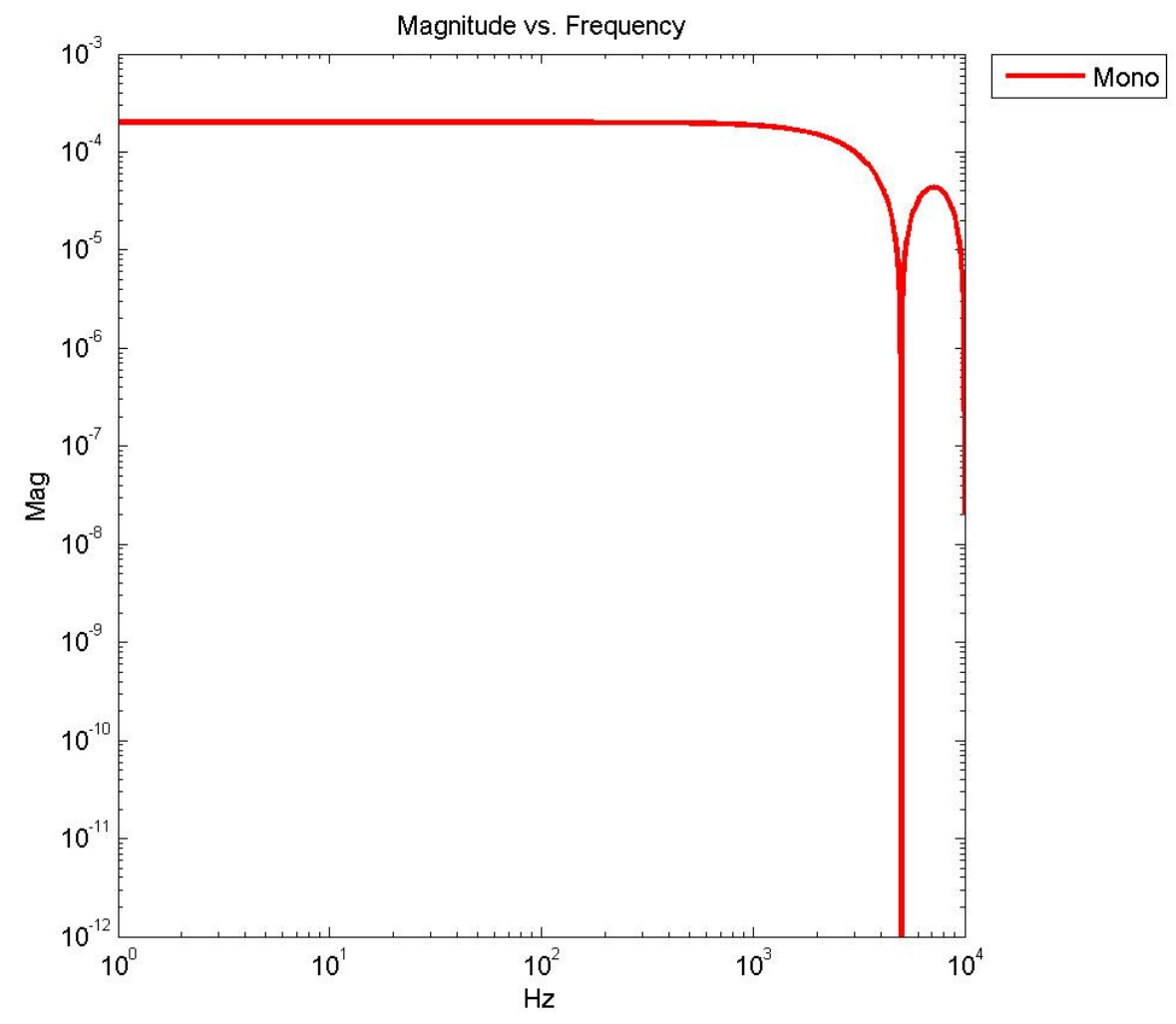

Figure 9: Magnitude response plot of a monophasic pulse with a pulse width, $\mathrm{T}=50 \mu \mathrm{s}$

Figure 9 shows the amount of signal content that a $50 \mu$ s monophasic pulse has from $1 \mathrm{~Hz}$ $-10 \mathrm{kHz}$. This type of analysis can be used to visually compare the amount of signal content within a desired frequency between various pulse types and pulse widths.

\section{Dipole-to-Dipole Interactions}

The interaction between the SCS device and ICD can be setup as a dipole-to-dipole interaction problem. The SCS device is emitting a voltage potential between two electrodes, anode (+) to cathode (-), which can act like the $+\mathrm{Q}$ and $-\mathrm{Q}$ in Figure 10. The distance, $\mathrm{L}$, is how far apart the two SCS electrodes are spaced. An SCS lead can have as many as 8 different electrodes, so the distance, $\mathrm{L}$, varies depending on which 2 electrodes are selected. The point $\mathrm{P}$, where we are measuring voltage potential, is the position of the ICD electrodes because it is the device that is sensing the voltage emitted from the SCS device. The value, $r$, is the distance between the ICD and SCS device. 


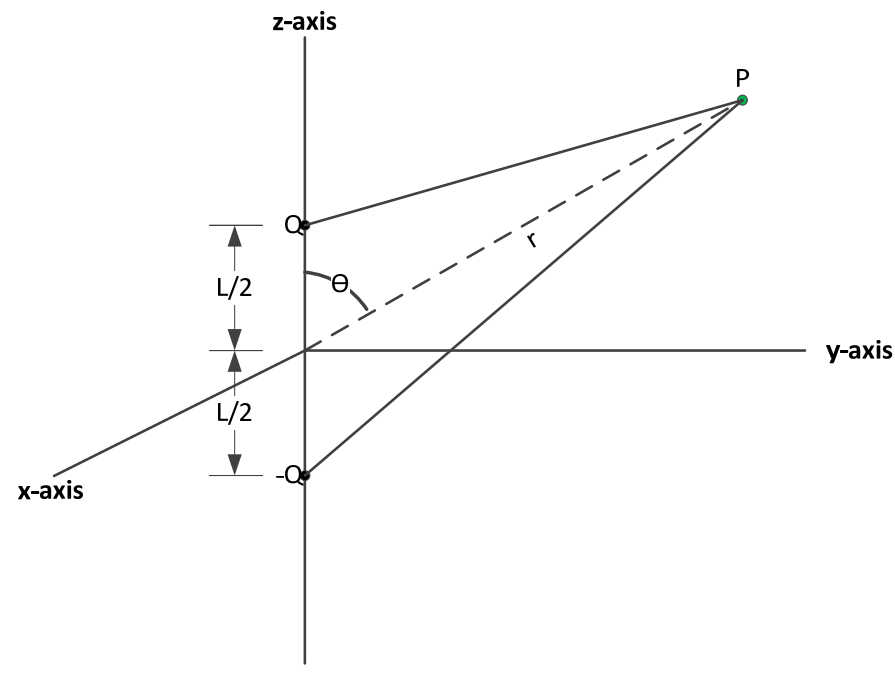

Figure 10: The electric dipole

Using a Cartesian coordinate system, we can configure the SCS to have its dipole moment along the z-axis, as shown in Figure 10, which illustrates the relationship between an electric dipole and the potential seen at point $P$ from that electric dipole. This potential voltage due to the dipole moment of the SCS device at point $\mathrm{P}, \mathrm{V}(\mathrm{r})$, can be calculated using the following equation:

$$
V(r)=(\Delta V \cdot L) \cdot\left(\frac{1}{\left|r-\frac{L}{2}\right|}-\frac{1}{\left|r+\frac{L}{2}\right|}\right)
$$

Where,

$$
\left|r \pm \frac{L}{2}\right|=\sqrt{(x)^{2}+(y)^{2}+\left(z \pm \frac{L}{2}\right)^{2}}
$$

The dipole model equates: $\mathrm{Q} /\left(4 \pi \epsilon_{0}\right)=\Delta \mathrm{V}^{*} \mathrm{~L}$ where $\Delta \mathrm{V}$ is the potential difference between the anode $(+\mathrm{Q})$ and cathode $(-\mathrm{Q})$ of the SCS device. This is calculated via superposition and assuming the poles are equal and opposite. The voltage potential sensed by the ICD is dependent on the distance between its two electrodes and can be expressed by the equation below:

$$
V_{I C D}=V\left(r_{1}\right)-V\left(r_{2}\right)
$$

In this equation, ' $\mathrm{r}_{1}$ ' and ' $\mathrm{r}_{2}$ ' represent the location of the two ICD electrodes relative to the center of the two SCS electrodes. Note: This does not factor in the sense filter internal to the ICD device. From the dipole-dipole model described above, we can conclude that the voltage potential sensed by the ICD is greater when its electrodes parallel $\left(0,180^{\circ}\right)$ to the SCS electrodes instead of perpendicular $\left(90,270^{\circ}\right)$. This will be an important setup condition when measuring the ICD voltage in vitro; placing the electrodes on the same axis will increase the amount of voltage sensed by the ICD electrodes. 


\section{E. Pulse Generator Design}

The pulse generator design involves 3 integral parts: A laptop, an ATMEL microcontroller $(\mu \mathrm{C})$, and a custom piece of hardware, "V-I Pulse Converter", that generates a constant current pulse based on signals sent by the $\mu \mathrm{C}$. This piece of hardware was designed by (Poore, 2012), an employee of St. Jude Medical. The laptop connected to the $\mu \mathrm{C}$ via a USB cable provides a $+3.3 \mathrm{~V}$ regulated power supply (converted from the $+5 \mathrm{~V}$ of the USB) and serial communication to the $\mu \mathrm{C}$. The $\mu \mathrm{C}$ has 4 signals that connect to the "V-I Pulse Converter" to control the different pulse parameters (shown in Figure 11).

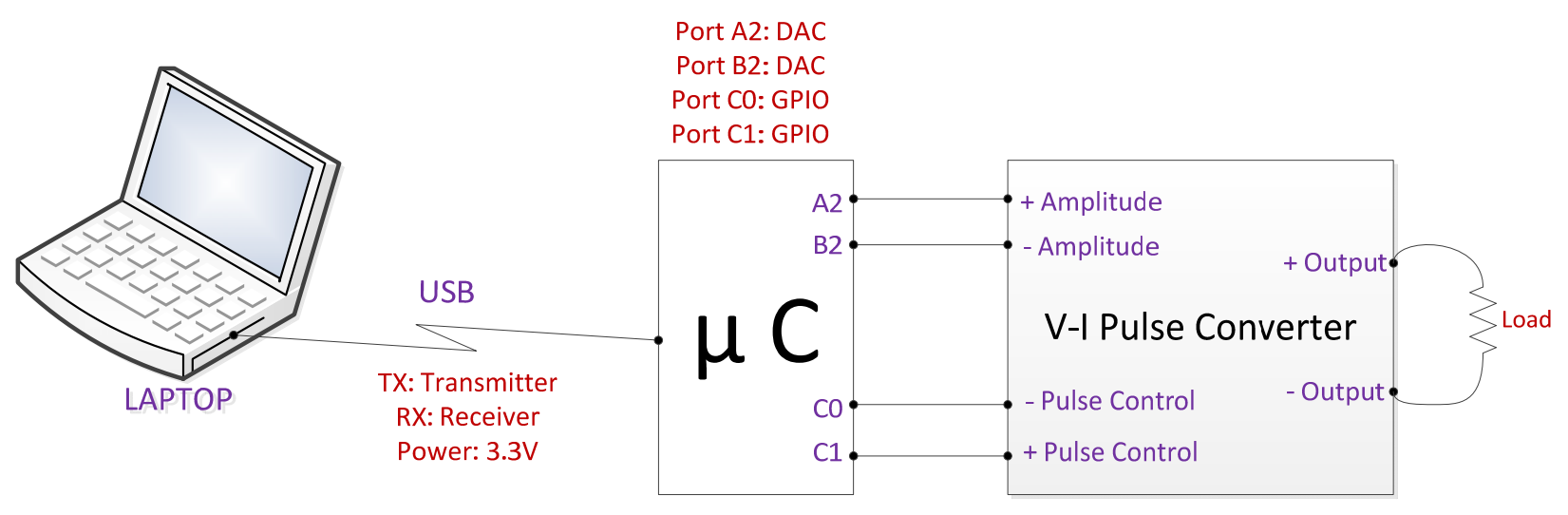

Figure 11: Pulse Generator Diagram

A graphical user interface (GUI) executable (“*.exe”), written in C\# (Visual Studios 2010), was created to allow the user to easily set the positive/negative pulse amplitude, pulse width, pulse period, and pulse type (Monophasic, Biphasic, etc.). This layer of code was operated from the Laptop computer and interfaced to the firmware running on the $\mu \mathrm{C}$. The $\mu \mathrm{C}$ firmware, written in C (Atmel Studios 6.0), was developed to control the "V-I Pulse Converter" based on the user settings communicated by the GUI executable running on the laptop. The "V-I Pulse Converter" operates on 4, 9V batteries, and generates a positive or negative constant current pulse based on a voltage reference and a digital output pin from the $\mu \mathrm{C}$. The positive and negative current pulses are controlled individually, which is why there are 4 connections needed from the $\mu \mathrm{C}$. Note: The grounds of the $\mu \mathrm{C}$ and the "V-I Pulse Converter" are connected together.

\section{Hardware}

This section references signals names and component designators from the "V-I Pulse Converter" schematic (Figure 42) which is located in Appendix E: Hardware Schematics. The circuit is powered by $4,9 \mathrm{~V}$ batteries that create $\mathrm{a}+18$ and $-18 \mathrm{~V}$ supply. The $\pm 18 \mathrm{~V}$ supplies are fed into two regulators ( $\mathrm{U} 1, \mathrm{U} 2)$ that create a regulated $\pm 16 \mathrm{~V}$ supply. The positive/negative 
current amplitudes are determined by two external voltage references, "Pos Curr Ref" and "Neg Curr Ref", and the value of the resistor used to sense the current. For this design, a resistance of $120 \Omega$ was used for the sense resistors (R11, R21).

Ohms Law: $I=\frac{V}{120}$

The output of the "V-I Pulse Converter" is controlled by two digital output pins, "Pos Curr Ctrl" and "Neg Curr Ctrl". When the output of the corresponding digital pin goes high, a pulse with amplitude 'I' will be sourced from "Pat Curr" (output) to ground. When the output of the digital pin goes low, a pulse will not be sourced from "Pat Curr" (output) to ground. It will instead be switched over to a constant internal load of $500 \Omega$. This type of design allows for extremely fast switching: $<50 \mathrm{~ns}$ (see DG419LDY datasheet). Based on the design, it is possible to connect both the positive and negative side of the circuit together when both "Curr Ctrl" pins are high. This was taken into account when developing the firmware for the $\mu \mathrm{C}$.

\section{Software}

As mentioned before, there are two pieces of software that were written to successfully operate the pulse generator:

1. Firmware: Code running on the $\mu \mathrm{C}$

2. GUI: Graphical user interface executable running on a laptop

The firmware is continuously running whenever the $\mu \mathrm{C}$ is powered up. Upon power up, the firmware sets the $\mu \mathrm{C}$ in a "default" state and waits for information from the GUI. The $\mu \mathrm{C}$ and GUI communicate through UART (universal asynchronous receiver/transmitter) communication which is connected through the USB port on the Laptop and $\mu \mathrm{C}$. Once it receives data from the GUI, the $\mu \mathrm{C}$ will set up and output a pulse based on the data it receives. The GUI is used to control all variable parameters of the pulse. It can also read back the parameters values to let the user know the state of the $\mu \mathrm{C}$. The GUI can be opened or closed at any time, and the $\mu \mathrm{C}$ will continue to run the last settings programed by the GUI as long as a power source is still supplied. 


\section{a. Firmware}

The firmware uses 6 global variables to allow the user to program 6 different pulse settings:

- "gl_State" = On/Off state of the pulse generator's output

- "gl_PulseWidth = Pulse width (in $\mu \mathrm{A})$

- "gl_PulsePeriod" = Pulse period (in ms)

- "gl_PulseType" = Pulse type (Mono, Bi, Tri, or Pentapolar)

- "gl_PulsePosAmp" = Positive pulse amplitude (in mA)

- "gl_PulseNegAmp" = Negative pulse amplitude (in mA)

There is also a variable designated for the pulse gap, "gl_PulseGap". This was intended to allow the user to vary the interphasic gap time; however, this feature was not implemented in the current code. The interphasic gap time was hardcoded to $1 \mu \mathrm{s}$.

When the microcontroller is first powered up, the firmware will initialize the 6 global variables to the following settings:

- "gl_State" = On

- “gl_PulseWidth $=200 \mu \mathrm{A}$

- "gl_PulsePeriod" $=20 \mathrm{~ms}$

- "gl_PulseType" = Biphasic

- “gl_PulsePosAmp" = 10mA

- “g1_PulseNegAmp" = 10mA

The variables, "gl_PulsePosAmp" and "gl_PulseNegAmp", will set two individual DACs that connect to the "Pos Curr Ref" and "Neg Curr Ref" respectively. The firmware internally converts the desired current to a voltage based on the "Ohms Law" equation (see Hardware section). Once these variables are initialized, the code begins in the "START" state. Figure 12 shows the 10 events and 10 states that were designed into this firmware code. 


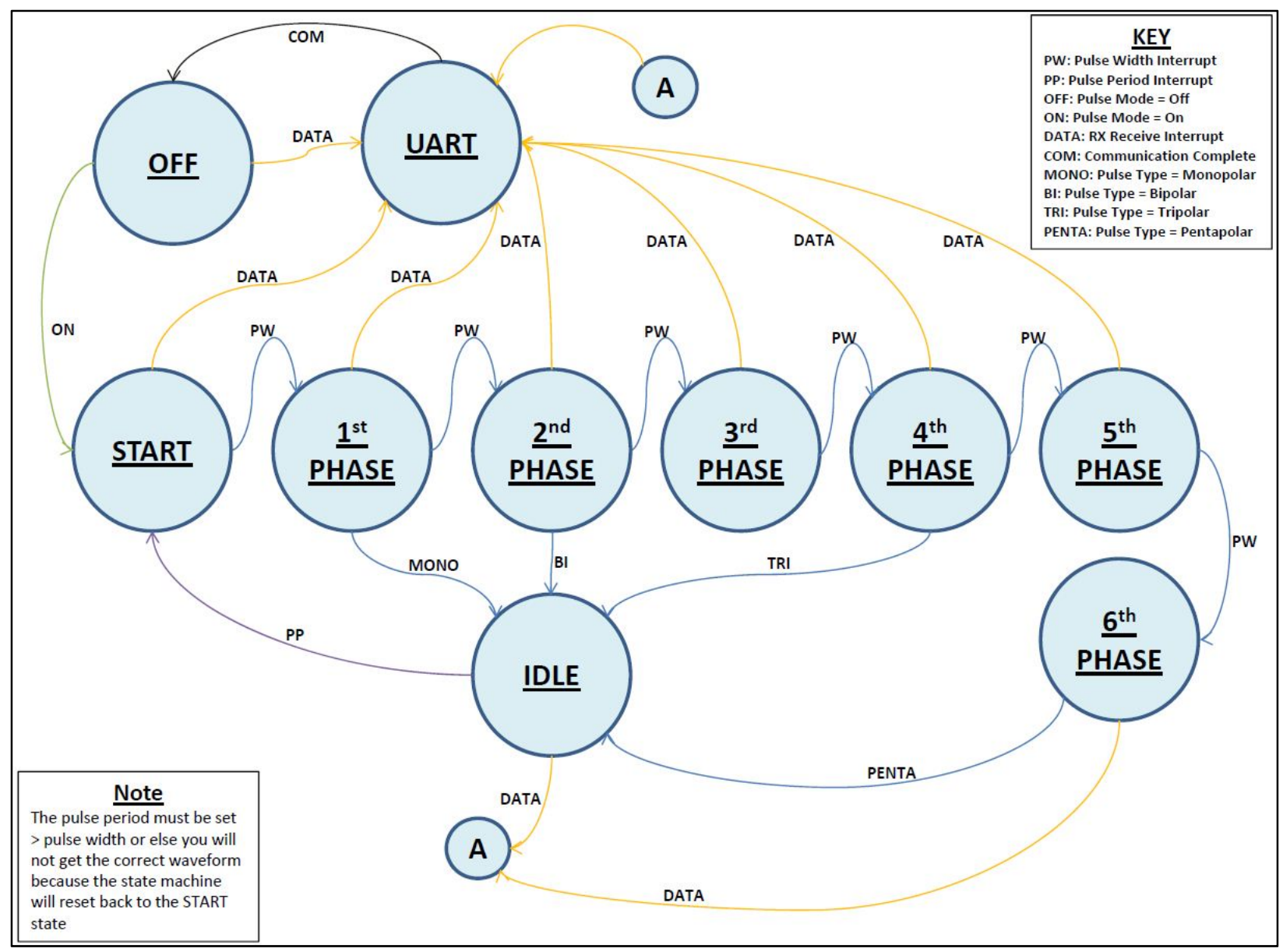

Figure 12: Microcontroller Firmware State Diagram

The different events are described below:

1. PW: This event is triggered when the "TIMER_WIDTH" timer interrupt is detected

a. The "TIMER_WIDTH" timer is set depending on the pulse type and pulse width

b. It does not always equal "gl_PulseWidth":

- For Monophasic, "TIMER_WIDTH" time $=2 *$ gl_PulseWidth

- For Biphasic, "TIMER_WIDTH" time = gl_PulseWidth

- For Triphasic, "TIMER_WIDTH” time = gl_PulseWidth $/ 2$

- For Pentapolar, "TIMER_WIDTH" time = gl_PulseWidth/2

2. PP: This event is triggered when the "TIMER_PERIOD" timer interrupt is detected

3. OFF: This event is triggered when the variable, "gl_State" $=0$.

4. ON: This event is triggered when the variable, "gl_State" $=1$.

5. DATA: This event is triggered when data has been received in the UART receive register

6. COM: This event is triggered when the UART communication has completed

7. MONO: This event is triggered when the variable, "gl_PulseType" $=1$.

8. BI: This event is triggered when the variable, "gl_PulseType" $=2$.

9. TRI: This event is triggered when the variable, "gl_PulseType" $=3$. 
10. PENTA: This event is triggered when the variable, "gl_PulseType" $=4$.

The different states are described below:

1. START
a. Sets current state $=$ START
b. Enables "TIMER_PERIOD" which starts it at time $=0$
c. Enables "TIMER_WIDTH" which starts it at time $=0$
d. Outputs a negative pulse (all pulses start negative)
e. Enables all interrupts

2. $1^{\text {st }}$ Phase
a. $\quad$ Sets current state $=$ PHASE 1
b. For a monophasic pulse, go to the "IDLE" state
c. For a biphasic pulse, outputs a positive pulse
d. For a triphasic pulse, outputs a positive pulse
e. For a pentapolar pulse, outputs a positive pulse

3. $2^{\text {nd }}$ Phase
a. $\quad$ Sets current state $=$ PHASE2
b. For a monophasic pulse, go to the "IDLE" state (this scenario should never occur)
c. For a biphasic pulse, go to the "IDLE" state
d. For a triphasic pulse, still output a positive pulse
e. For a pentapolar pulse, disable the output

4. $3^{\text {rd }}$ Phase
a. Sets current state $=$ PHASE3
b. For a monophasic pulse, go to the "IDLE" state (this scenario should never occur)
c. For a biphasic pulse, go to the "IDLE" state (this scenario should never occur)
d. For a triphasic pulse, outputs a negative pulse
e. For a pentapolar pulse, output remains disabled

5. $4^{\text {th }}$ Phase

a. $\quad$ Sets current state $=$ PHASE 4

b. For a monophasic pulse, go to the "IDLE" state (this scenario should never occur)

c. For a biphasic pulse, go to the "IDLE" state (this scenario should never occur)

d. For a triphasic pulse, go to the "IDLE" state

e. For a pentapolar pulse, output a positive pulse

6. $5^{\text {th }}$ Phase

a. $\quad$ Sets current state $=$ PHASE5

b. For a monophasic pulse, go to the "IDLE" state (this scenario should never occur)

c. For a biphasic pulse, go to the "IDLE" state (this scenario should never occur)

d. For a triphasic pulse, go to the "IDLE" state (this scenario should never occur)

e. For a pentapolar pulse, output a negative pulse

7. $6^{\text {th }}$ Phase 
a. Sets current state $=$ PHASE6

b. For a monophasic pulse, go to the "IDLE" state (this scenario should never occur)

c. For a biphasic pulse, go to the "IDLE" state (this scenario should never occur)

d. For a triphasic pulse, go to the "IDLE" state (this scenario should never occur)

e. For a pentapolar pulse, go to the "IDLE" state

8. IDLE
a. Sets current state $=$ IDLE
b. Disables the "TIMER_WIDTH" timer
c. Disables the output

9. UART (see Table 1 for "Command Code" and "Parameter Code" descriptions)

a. Sets current state $=$ IDLE

b. Disables the output

c. Reads the "Command Code" byte

d. Sends the "Command Code" byte back (for error checking)

e. Reads the "count" byte (\# of bytes remaining to be read, not including this byte)

f. Reads the number of bytes specified by the "count" byte

i. $1^{\text {st }}$ byte = "Parameter Code"

ii. $2^{\text {nd }}$ byte $-2^{\text {nd }}$ from last byte $=$ Value

iii. Last byte $=$ "response" command (for error checking)

g. If the "Command Code" = 'A'

i. The value ( 4 bytes) is converted to a float and the appropriate variable is updated based on the "Parameter Code"

ii. Updated variable value it is stored in a temporary variable, "d_value"

iii. The "response" is set to 'ACK' (acknowledge)

h. If the "Command Code" = 'B'

i. The appropriate variable, based on the "Parameter Code", is read and stored in "d_value"

ii. The "response" is set to 'ACK' (acknowledge)

i. If the "Command Code" $=$ 'C'

i. All the global variables are converted back to their default (power up) values

ii. "d_value" is set to 0 and "response" is set to "ACK' (acknowledge)

j. Responds back with the variables "d_value", "error", and "response"

i. $1^{\text {st }}$ byte = "response"

ii. $2^{\text {nd }}-5^{\text {th }}$ bytes $=$ "d d value"

iii. $6^{\text {th }}$ byte $=$ error

k. Clears the RX (receive) and TX (transmit) complete interrupts

1. Goes to the "OFF" state

10. OFF

a. Disables all interrupts 
b. Sets current state $=\mathrm{OFF}$

c. Clears all/any pending events

d. If "gl_State" =1, then go to "START" state

Table 1: UART Command and Parameter Code Descriptions

\begin{tabular}{clcl} 
Command Code & Description & Parameter Code & Description \\
\hline 'A' & Set Pulse Parameters & 1 & Pulse Width \\
'B' & Get Pulse Parameters & 2 & Pulse Gap (not used) \\
'C' & Revert to defaults & 3 & Pulse Period \\
& & 4 & Pulse Type \\
& & 5 & + Pulse Amp \\
& & 6 & - Pulse Amp \\
\hline
\end{tabular}

One additional transition, not shown in Figure 12, is the firmware will transition to the "START" state whenever a "PP" event is detected. This scenario will occur if the pulse period < pulse width. This situation is taken care of by the GUI. The GUI does not allow for a pulse period < pulse width to be input by the user. The actual code is listed in Appendix C: Pulse Generator Firmware Code.

\section{b. Graphical User Interface (GUI)}

As mentioned previously, the graphical user interface executable communicates with the $\mu \mathrm{C}$ via a USB-UART connection. The GUI is shown in Figure 13. The program performs 5 basic functions:

1. Initiate a UART connection

a. Connects the computer to the $\mu \mathrm{C}$ via the correct serial communication port ("Com Port")

b. The BAUD rate is hardcoded in the $\mu \mathrm{C}$ to $9600 \mathrm{bps}$ (bits per second) so 9600 , is the only BAUD setting that will successfully connect to the $\mu \mathrm{C}$.

c. The Com Port can vary, so this is programmable. Once the correct Com Port is found, hitting enter on the keyboard will attempt to connect the computer to the $\mu \mathrm{C}$.

d. If the connection is successful, "Device Connected" will display on the bottom of the GUI.

e. If the connection is not successful, "Device Not Connected" will display on the bottom of the GUI.

2. Set Pulse Parameters

a. Records the "Pulse Type", "Pulse Width", "Pulse Period", "+ Pulse Amp", and "Pulse Amp" values set on the GUI 
b. For each of the values in step a, send "Command Code" ' $A$ ' and the value recorded from the GUI to the $\mu \mathrm{C}$ to update that parameter

c. Record the response back from the $\mu \mathrm{C}$, including the value the $\mu \mathrm{C}$ received and update the corresponding value on the GUI

3. Get Pulse Parameters

a. For the "Pulse Type", "Pulse Width", "Pulse Period", "+ Pulse Amp", “- Pulse Amp", and "ON/OFF" values, send "Command Code" 'B' and record the response back from the $\mu \mathrm{C}$

b. Update the corresponding value on the GUI based on the value returned by the $\mu \mathrm{C}$

4. Revert To Defaults

a. Send "Command Code" 'C'

b. Call "Get Pulse Parameters" function to update GUI

5. $\mathrm{ON} / \mathrm{OFF}$

a. If the "ON/OFF" button text = "OFF"

i. Send "Command Code" 'A' with a value of 1 to turn on the output

ii. Update the "ON/OFF" button text $=$ "ON" and a color of green

b. If the "ON/OFF" button text = "ON"

i. Send "Command Code" "A' with a value of 0 to turn off the output

ii. Update the "ON/OFF" button text = "OFF" and a color of grey

To guarantee the user could not input values greater than the variables allowable range, maximum and minimum constraints were put on the GUI's variables:

- Pulse Type

o Available Options: Monophasic, Biphasic, Triphasic, or Pentapolar

- Pulse Width $(\mu s)$

$$
\begin{array}{ll}
\text { o } & \text { Min }=200 \\
\text { o } & \text { Max }=1000 \\
\text { o } & \text { Min Step Size = 1 }
\end{array}
$$

- Pulse Gap (us) - not used

- Pulse Period (ms)

o $\quad \operatorname{Min}=10$

o $\operatorname{Max}=2000$

o $\quad$ Min Step Size $=1$

- + Pulse Amp (mA)

o Min $=0.00$

o $\operatorname{Max}=30.00$

o Min Step Size $=0.01$

- - Pulse Amp (mA)

o $\quad \operatorname{Min}=0.00$

o $\operatorname{Max}=30.00$ 
o Min Step Size $=0.01$

The actual code is listed in Appendix D: GUI Executable Code.

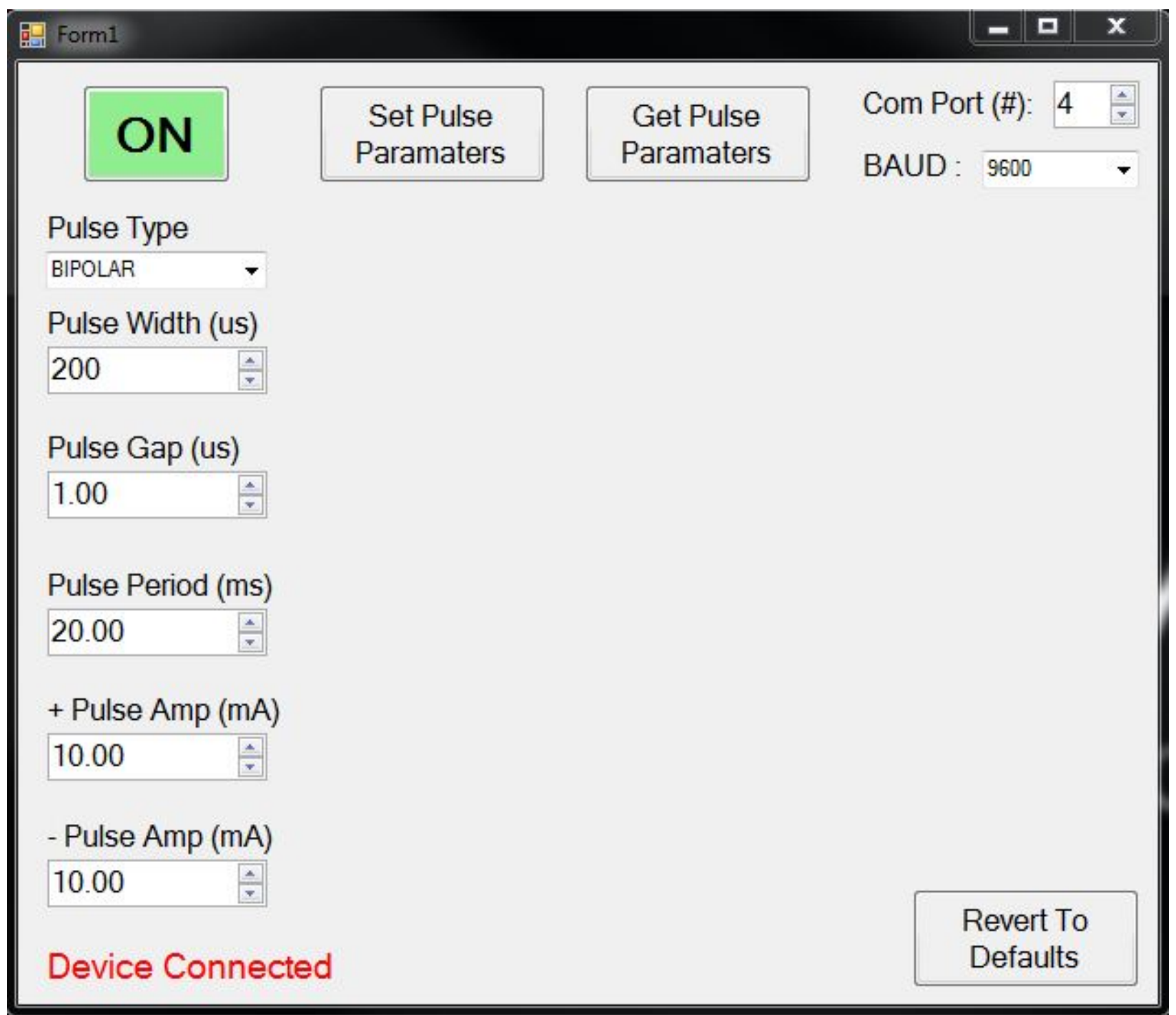

Figure 13: Pulse Generator GUI Executable

\section{Methods}

The goal of this research is to show analytically and in vitro that a triphasic pulse, when compared to a monophasic and biphasic pulse, will decrease the amount of crosstalk between an SCS and an ICD device that is implanted in the same person. The analytical experiment was performed in Matlab based on the details described in the Analytical Experimental Method section. The in vitro experiment was performed in a saline solution using a pulse generator specially designed for this experiment. The pulse generator design was verified based on the 
details described in the Pulse Generator Verification section. The saline experiment was performed based on the details described in the Saline Experimental Method section.

The ICD specifications used in these experiments were from the Fortify Assura ${ }^{\mathrm{TM}}$ family; the latest ICD device from St. Jude Medical. The SCS specifications were based on the Eon Mini $^{\mathrm{TM}}$; the latest SCS device from St. Jude Medical. Both of these devices specifications can be found online at St. Jude Medical's website.

\section{A. Analytical Experimental Method}

To model the different pulse waveforms in Matlab, each pulse's $\mathrm{f}(\mathrm{t})$, function vs. time, was determined using an arbitrary period, T. An ideal monophasic waveform, shown in Appendix A: Pulse Generator Waveforms, has 1 phase from time $-\mathrm{T} / 2$ to $\mathrm{T} / 2$. Using a unit step function, this can be modeled by the function:

$$
f(t)=-u\left(t-\frac{T}{2}\right)+u\left(t+\frac{T}{2}\right)
$$

An ideal biphasic waveform, shown in Appendix A: Pulse Generator Waveforms, has 2 phases:

- A negative phase from time - T to 0

- A positive phase from time 0 to $\mathrm{T}$

Using a unit step function, this can be modeled by the function:

$$
f(t)=-u(t+T)+2 u(t)-u(t-T)
$$

An ideal triphasic waveform, shown in Appendix A: Pulse Generator Waveforms, has 3 phases:

- A negative phase from time $-2 \mathrm{~T}$ to $-\mathrm{T}$

- A positive phase from time - $\mathrm{T}$ to $\mathrm{T}$

- A negative phase from time $\mathrm{T}$ to $2 \mathrm{~T}$

Using a unit step function, this can be modeled by the function:

$$
f(t)=-u(t+2 T)+2 u(t+T)-2 u(t-T)+u(t-2 T)
$$

An ideal pentapolar waveform, shown in Appendix A: Pulse Generator Waveforms, has 5 phases:

- A negative phase from time $-3 \mathrm{~T}$ to $-2 \mathrm{~T}$

- A positive phase from time $-2 \mathrm{~T}$ to $\mathrm{T}$

- A zero phase from time $-\mathrm{T}$ to $\mathrm{T}$ 
- A positive phase from time $\mathrm{T}$ to $2 \mathrm{~T}$

- A negative phase from time $2 \mathrm{~T}$ to $3 \mathrm{~T}$

Using a unit step function, this can be modeled by the function:

$$
f(t)=-u(t+3 T)+2 u(t+2 T)-u(t+T)+u(t-T)-2 u(t-2 T)+u(t-3 T)
$$

There is a built-in $1 \mu$ s delay in the firmware of the pulse generator to account for the physical switching time of the hardware. A triphasic pulse with an interphasic gap of ' $D$ ', shown in Appendix A: Pulse Generator Waveforms, can be expressed by using 5 phases:

- A negative phase from time $-(2 \mathrm{~T}+\mathrm{D})$ to $-(\mathrm{T}+\mathrm{D})$

- A zero phase from time $-(\mathrm{T}+\mathrm{D})$ to $-\mathrm{T}$

- A positive phase from time -T to $\mathrm{T}$

- A zero phase from time $\mathrm{T}$ to $\mathrm{T}+\mathrm{D}$

- A negative phase from time $\mathrm{T}+\mathrm{D}$ to $2 \mathrm{~T}+\mathrm{D}$

Using a unit step function, this can be modeled by the function:

$$
\begin{aligned}
f(t)=-u(t & +(2 T+D))+u(t+(T+D))+u(t+T)-u(t-T)-u(t-(T+D)) \\
& +u(t-(2 T+D))
\end{aligned}
$$

Then, the $5 \mathrm{f}(\mathrm{t})$ equations were converted into a $\mathrm{F}(\mathrm{s})$ using the Laplace (see the Laplace Transform section):

$$
\begin{aligned}
& F_{\text {mono }}(s)=\frac{1}{s}\left[e^{\left(\frac{T}{2}\right) s}-e^{-\left(\frac{T}{2}\right) s}\right] \\
& F_{b i}(s)=\frac{1}{s}\left[-e^{T s}+2-e^{-T s}\right] \\
& F_{\text {tri }}(s)=\frac{1}{s}\left[-e^{2 T s}+2 e^{T s}-2 e^{-T s}+e^{-2 T s}\right] \\
& F_{\text {tri_delay }}(s)=\frac{1}{s}\left[-e^{(2 T+D) s}+e^{(T+D) s}+e^{T s}-e^{-T s}-e^{-(T+D) s}+e^{-(2 T+D) s}\right] \\
& F_{\text {penta }}(s)=\frac{1}{s}\left[-e^{3 T s}+2 e^{2 T s}-e^{T s}+e^{-T s}-2 e^{-2 T s}+e^{-3 T s}\right]
\end{aligned}
$$

Recall: $s=j 2 \pi f$

The F(s) equations were all converted into Matlab code (“*.m” file). Refer to the Frequency Domain Functions section in Appendix B: Matlab Code for the code. The inputs to each function:

- "freq" = Frequency

- "T" = Pulse Width 
The outputs to the function:

- $\mathrm{F}=\mathrm{F}(\mathrm{s})$ in $\mathrm{A}+\mathrm{jB}$ form

- $\mathrm{M}=$ Magnitude

- $\mathrm{P}=$ Phase in degrees

Lastly, two pieces of test code, written in Matlab, used the Frequency Domain Functions to graphically compare the following cases:

1. All 4 waveform's magnitude response from 1 to $250 \mathrm{~Hz}$
a. $\mathrm{T}=50 \mu \mathrm{s}$
b. $\mathrm{T}=200 \mu \mathrm{s}$
c. $\mathrm{T}=500 \mu \mathrm{s}$

2. All 4 waveform's magnitude response from 1 to $10 \mathrm{kHz}$

a. $\mathrm{T}=50 \mu \mathrm{s}$

3. Triphasic waveform's magnitude response with an interphasic delay, ' $\mathrm{D}$ ' $=0,1$, and $10 \mu \mathrm{s}$

a. $\mathrm{T}=50 \mu \mathrm{s}$

Refer to the Matlab Test Code section in Appendix B: Matlab Code for the Matlab test code. This experiment assumes an ideal waveform shape with instantaneous phase switching: This means no rise or fall times. A pulse width of $\mathrm{T}=50 \mu$ s and a frequency range of $1-250 \mathrm{~Hz}$ and 1$10 \mathrm{kHz}$ were selected to match the calculations performed in patent \#US20100331921 (Bharmi, Bornzin, \& Poore, 2010). A pulse width of $\mathrm{T}=200 \mu$ s and $500 \mu$ s were selected to match the saline experiments. All of the waveforms were normalized to 1 for comparison purposes.

\section{B. Saline Experimental Method}

A large empty tank will be filled with a $1.90 \mathrm{mS} / \mathrm{cm}(\sim 526 \Omega-\mathrm{cm})$ saline solution (Moore, 1972 ) to simulate the conductance of human body tissue (Geddes \& Baker, 1989). A SCS octal lead will be submerged into the saline solution and placed $9.1 \mathrm{~cm}$ away from a submerged IS-1 BI ICD lead. The electrodes will be on the same axis (i.e. $0^{\circ}$ or $180^{\circ}$ ) to get the largest crosstalk effect (see the Dipole-to-Dipole Interactions section). The output of the pulse generator will then be connected to the $1^{\text {st }}$ and $8^{\text {th }}$ electrode on the octal SCS lead. The $1^{\text {st }}$ and $8^{\text {th }}$ electrodes are spaced the furthest from one another and will be used to get the largest SCS dipole distance. A recording oscilloscope and two differential scope probes will be connected to the output of the pulse generator and the ICD leads to record the waveforms. Once the waveforms are recorded, the data from the ICD leads will then be processed through a Matlab filter that mimics a typical ICD's sense filter. See ICD Sense Filter in Appendix B: Matlab Code for the Matlab code. 


\section{Pulse Generator Verification}

The pulse generator design was split up into three parts: A GUI running on a laptop, a microcontroller $(\mu \mathrm{C})$, and a pulse generator. Based on inputs from the GUI, the pulse generator could vary its pulse period, pulse width, pulse amplitude, and its pulse type. To verify that the design of the pulse generator was done correctly, a $500 \Omega$ load will be connected across the output of the pulse generator and all of the variable inputs will be measured over a range that exceeds their use in the saline experiment:

- Pulse Period: A recording oscilloscope will be used to measure the pulse period at 10$50 \mathrm{~Hz}$ in $10 \mathrm{~Hz}$ steps.

- Pulse Width: A recording oscilloscope will be used to measure the pulse width at 200$1000 \mu$ s in $200 \mu$ s steps.

- Pulse Type: A recording oscilloscope and Excel will be used to capture each pulse type.

The positive/negative amplitude will be measured across the $120 \Omega$ sense resistor on the "V-I Pulse Converter". The input on the GUI will range from $2-14 \mathrm{~mA}$ in $4 \mathrm{~mA}$ steps. This is a more stable measurement than trying to measure the output pulse amplitude using the oscilloscope. The data will be recorded and analyzed in excel to calculate the pulse generator's percent error when compared to the requested value on the GUI, viz

$$
\% \text { Error }=\frac{\text { Desired }- \text { Actual }}{\text { Desired }} * 100
$$

\section{Saline Test}

Figure 14 shows a simplistic flow-diagram of the saline experiment test setup. Below is a description of the important items:

- SCS Electrodes: The $1^{\text {st }}$ and $8^{\text {th }}$ electrodes of an octal lead (8 total electrodes) are connected to the positive and negative terminal of the pulse generator's output (see the Pulse Generator Design section). The other end of the lead is placed in the saline solution where the $1^{\text {st }}$ electrode is $9.1 \mathrm{~cm}$ from the tip electrode on the ICD lead. Note: The electrode-terminal configuration (+/-) does not matter.

- ICD Electrodes: One side of the IS-1 BI lead (2 total electrodes) is connected to a differential amplifier and the other side is placed in the saline solution.

- $\quad$ CH. 1: A differential scope probe is connected from the output of the pulse generator to the "Ch. 1" input on a recording oscilloscope.

- CH. 2: A differential scope probe is connected from one side (the dry side) of the ICD lead to the "Ch. 2" input on a recording oscilloscope.

- Digital Oscilloscope: Setup to record both the SCS and ICD waveforms at the same point in time and dump the data into a "*.csv" file. 
- Sense Filter: Processes the ICD waveform through a filter that mimics the ICD's sense filter allowing for a comparison between the different pulse waveforms.

The data for the ICD and SCS waveforms will be recorded for each of the 4 pulse types (monophasic, biphasic, triphasic, and pentapolar) using the following settings:

1. Experiment \#1 Settings
a. Pulse Period $=50 \mathrm{~ms}$
b. Pulse Width $=200 \mu \mathrm{s}$
c. + Pulse Amplitude $=10 \mathrm{~mA}$
d. - Pulse Amplitude $=10 \mathrm{~mA}$

2. Experiment \#2 Settings
a. Pulse Period $=50 \mathrm{~ms}$
b. Pulse Width $=500 \mu \mathrm{s}$
c. + Pulse Amplitude $=10 \mathrm{~mA}$
d. - Pulse Amplitude $=10 \mathrm{~mA}$ 


\section{Saline Test Setup}

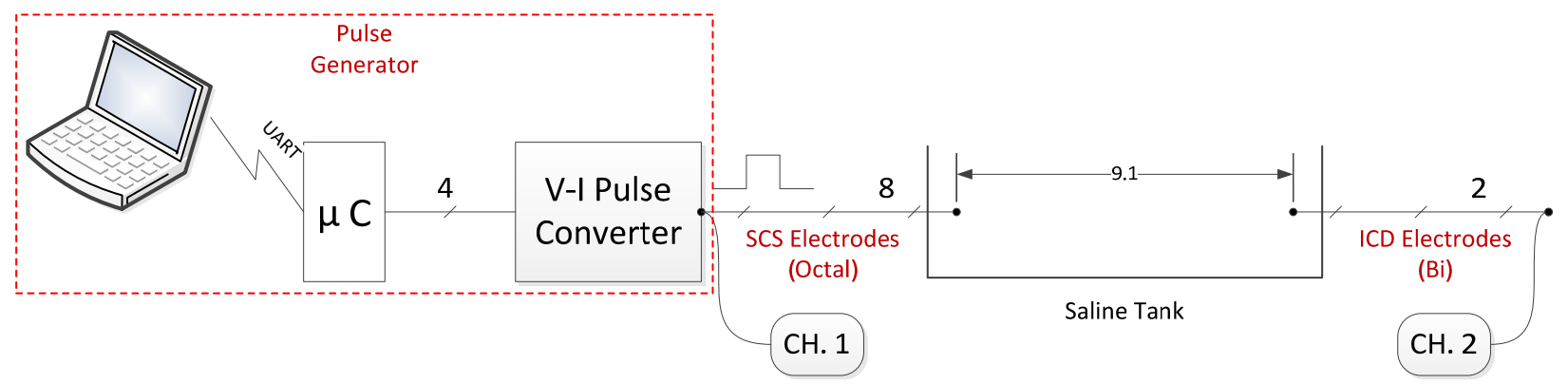

Data Processing

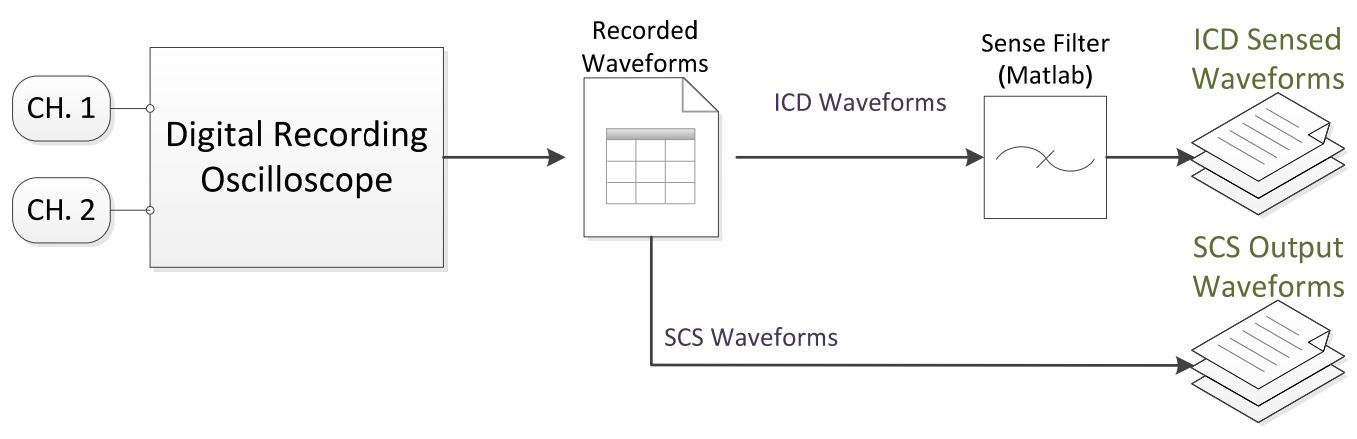

Figure 14: General flow outline of the saline experiment and data post-processing. 


\section{Results}

The procedure from the Methods section was followed to obtain the data in this section.

\section{A. Analytical}

The procedure from the Analytical Experimental Method section was followed to obtain all the figures in this section. The figures in this section compare the different waveform's magnitude (expressed in $\mathrm{dB}$ ) over various frequency ranges; this is known as the magnitude response. Refer to the legend in the top right hand corner of each figure to determine which waveforms are being compared.

Figure 15, Figure 16, and Figure 17 have a frequency range of $1-250 \mathrm{~Hz}$ at $\mathrm{T}=50,200$, and $500 \mu$ s respectively. Figure 18 has a frequency range of $1-10 \mathrm{~Hz}$ at $\mathrm{T}=50 \mu \mathrm{s}$. Figure 19 has a frequency range of $1-250 \mathrm{~Hz}$ at $\mathrm{D}=0,1$, and $10 \mu \mathrm{s}$. Figure 20 shows a zoomed in version of Figure 19 and has a frequency range of $100-110 \mathrm{~Hz}$.

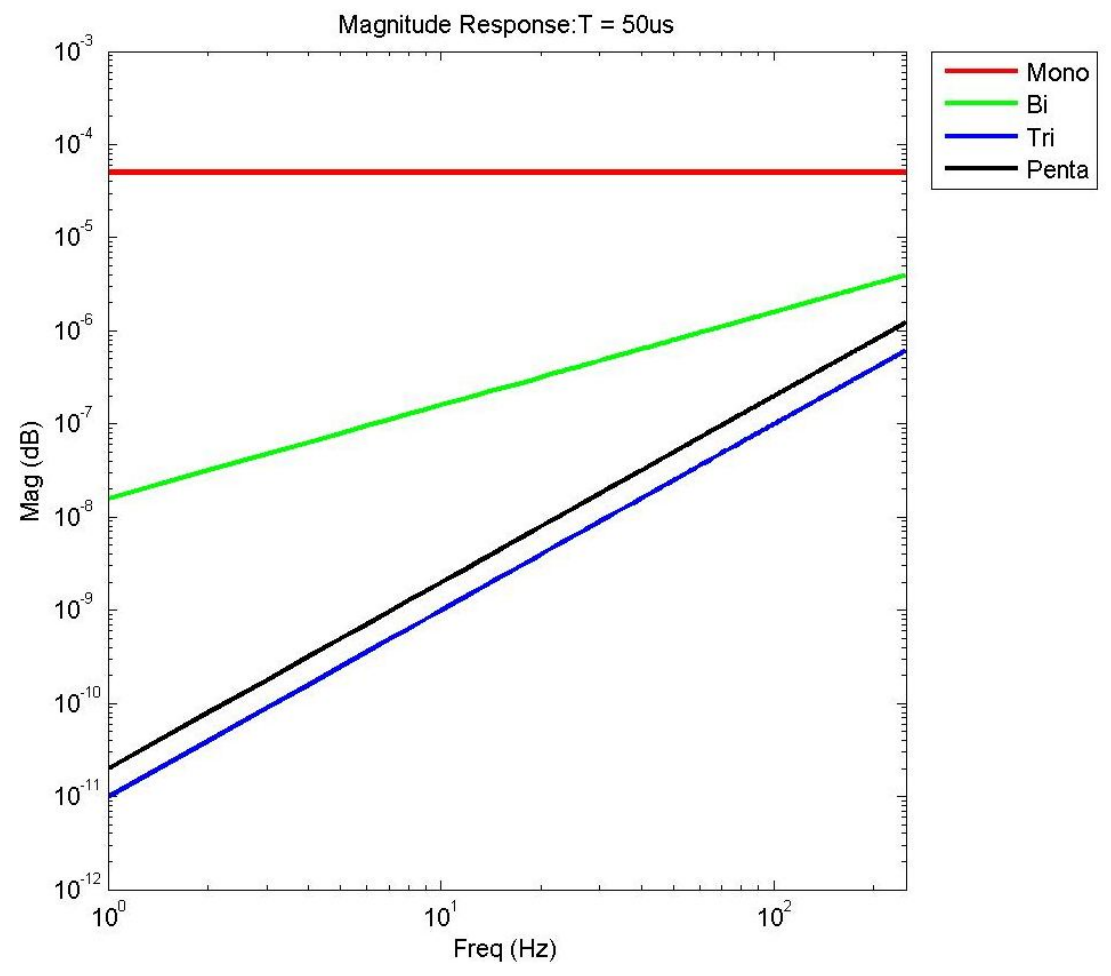

Figure 15: Magnitude response at $\mathrm{T}=50 \mu$ s with no interphasic gap 


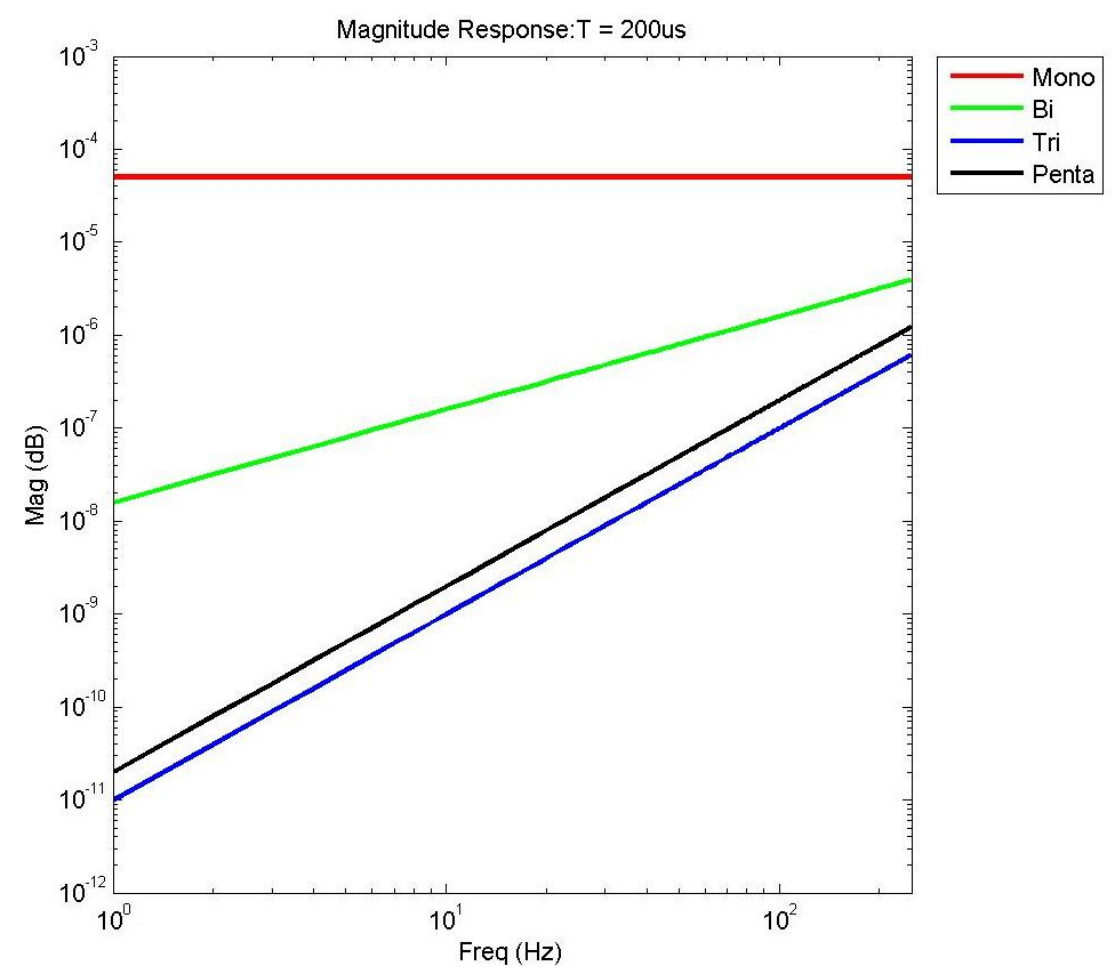

Figure 16: Magnitude response at $T=200 \mu$ s with no interphasic gap

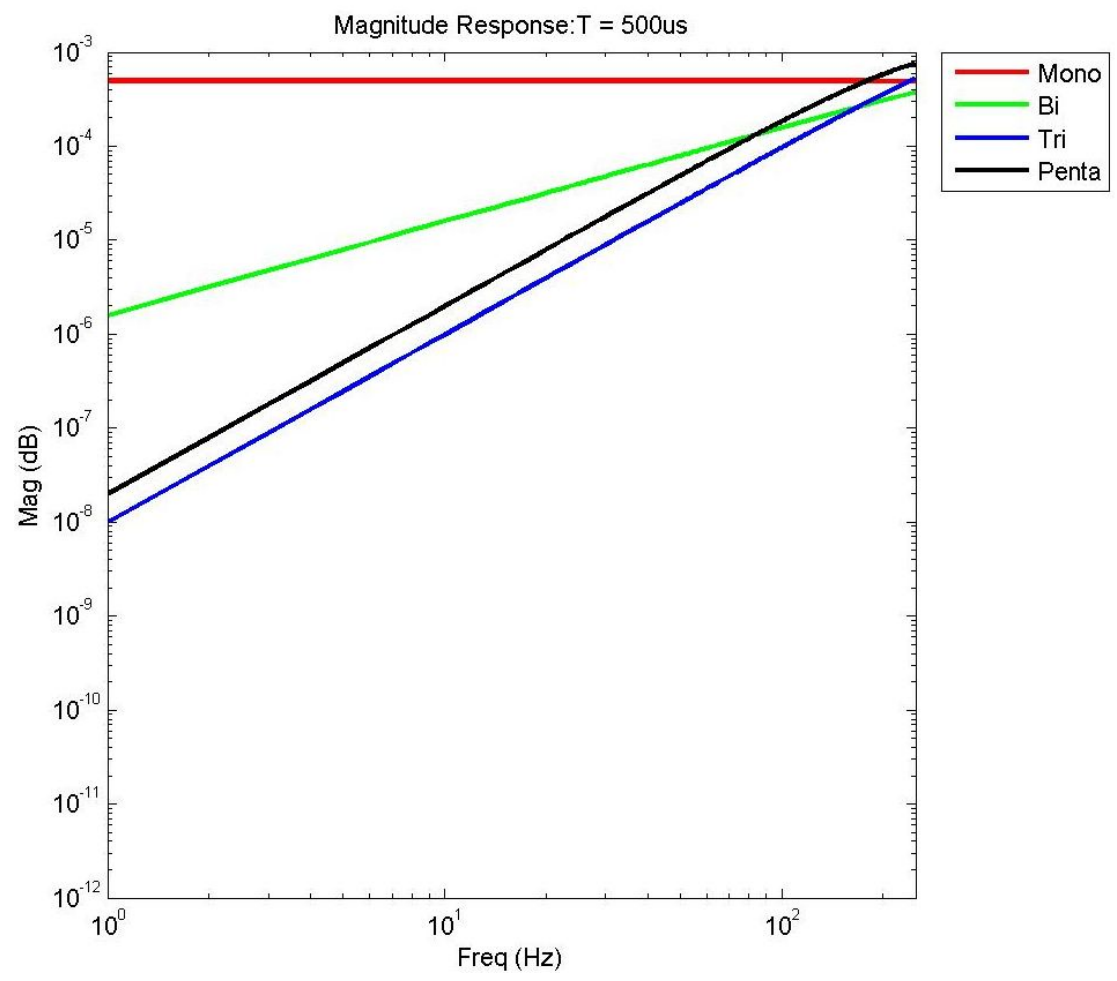

Figure 17: Magnitude response at $T=500 \mu$ s with no interphasic gap 


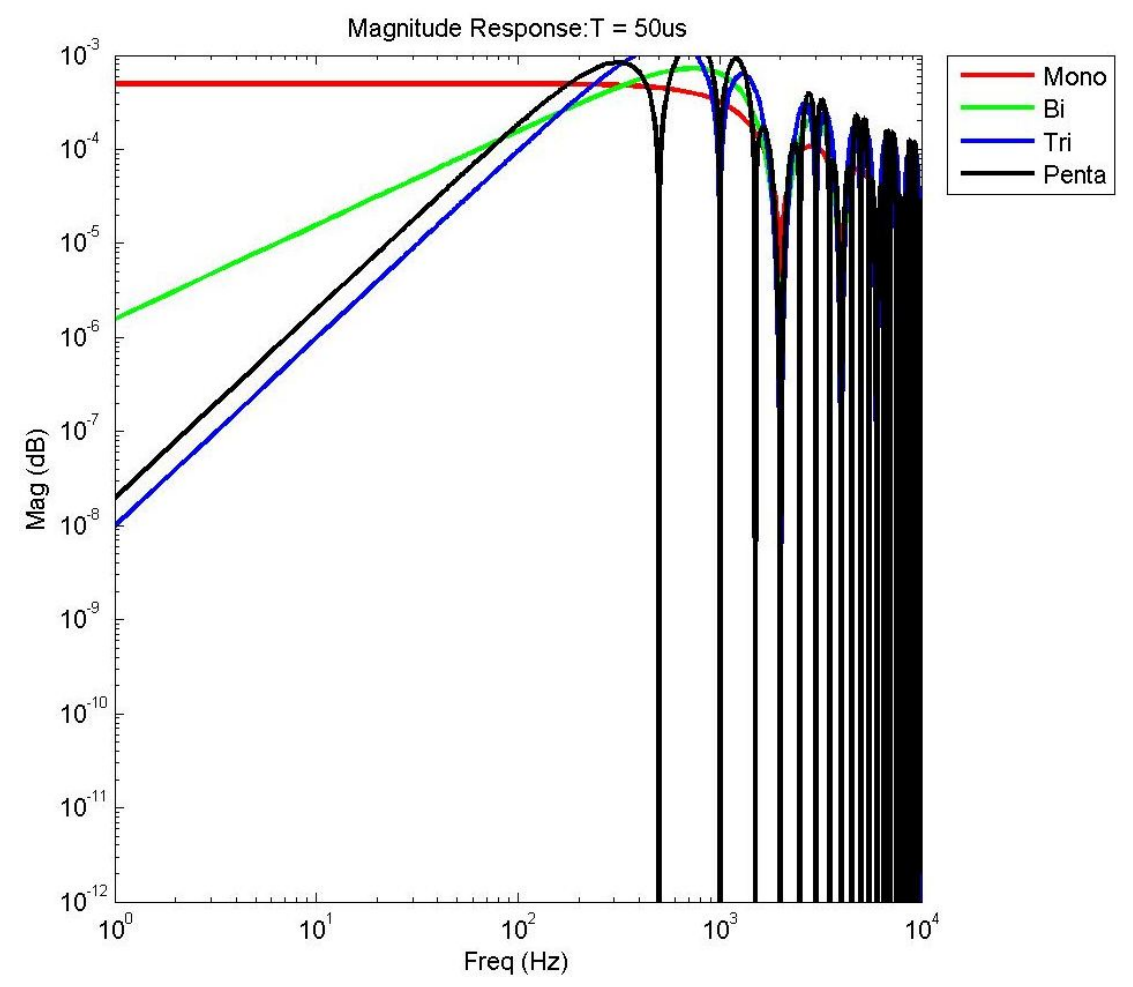

Figure 18: Magnitude response at $\mathrm{T}=50 \mu \mathrm{s}$ with no interphasic gap (zoomed out)

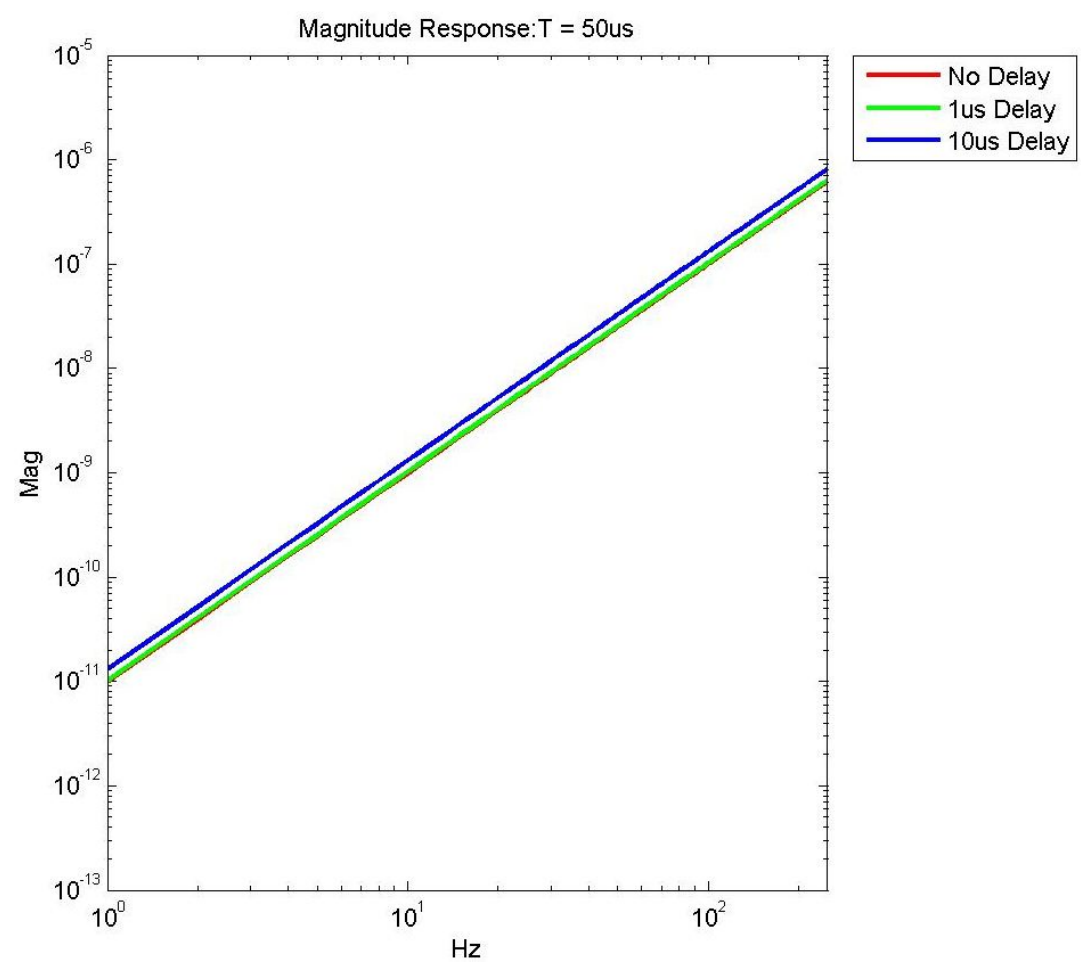

Figure 19: Magnitude response for a Triphasic waveform at $T=50 \mu$ s with an interphasic gap of 0 , 1 , and $10 \mu \mathrm{s}$ 


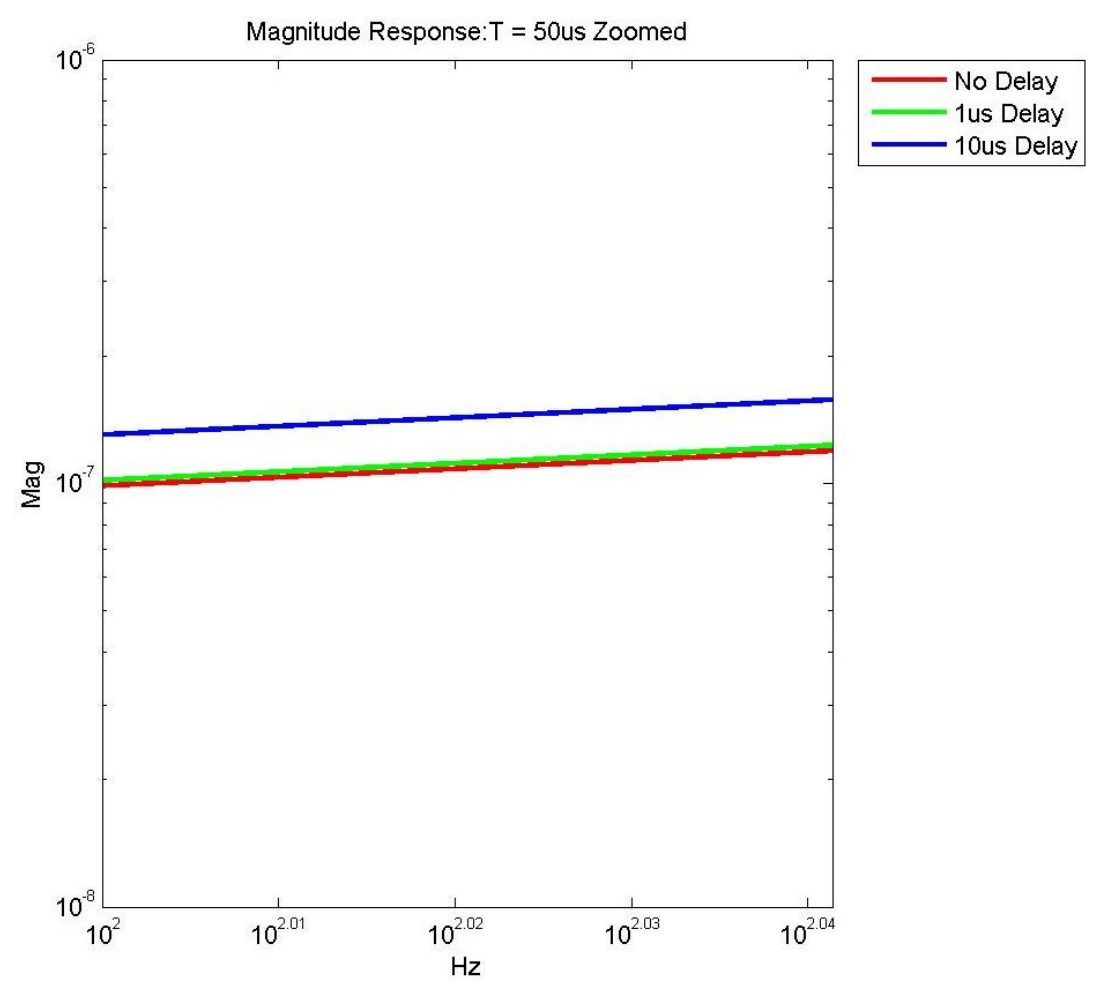

Figure 20: Zoomed in plot of Figure 19 at $100-110 H z$

\section{B. In Vitro}

The procedure from the Saline Experimental Method section was followed to obtain all the figures and tables in this section.

\section{Pulse Generator Verification}

A recording oscilloscope with a differential probe or an SMU (see Appendix F: Equipment for equipment details) was used to record all measurements and Excel was used for

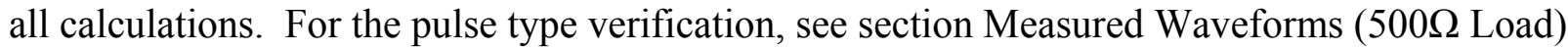
in Appendix A: Pulse Generator Waveforms for the pulse generator waveforms. Table 2, Table 3, Table 4, and Table 5 lists the selected value on the GUI, the measured value from the equipment, and the percent error which relates the two values. A $0 \%$ error is ideal.

Table 2: Pulse Period (Measured vs. Requested)

\begin{tabular}{cllc}
\hline $\begin{array}{l}\text { GUI } \\
\text { Pulse } \\
\begin{array}{l}\text { Period } \\
(\mathbf{m s})\end{array}\end{array}$ & $\begin{array}{l}\text { GUI } \\
\text { Pulse } \\
\text { Period } \\
(\mathbf{H z})\end{array}$ & $\begin{array}{l}\text { Measured } \\
\text { Pulse Period } \\
(\mathbf{m s})\end{array}$ & \%Error \\
\hline $\mathbf{2 0 . 0 0}$ & 50 & 20.00 & $0.00 \%$ \\
\hline
\end{tabular}




\begin{tabular}{cccc}
\hline $\mathbf{2 5 . 0 0}$ & 40 & 25.00 & $0.00 \%$ \\
\hline $\mathbf{3 3 . 3 3}$ & 30 & 33.20 & $0.40 \%$ \\
\hline $\mathbf{5 0 . 0 0}$ & 20 & 49.80 & $0.40 \%$ \\
$\mathbf{1 0 0 . 0 0}$ & 10 & 99.80 & $0.20 \%$ \\
\hline
\end{tabular}

Table 3: \pm Pulse Width (Measured vs. Requested)

\begin{tabular}{ccccc}
\hline $\begin{array}{c}\text { GUI } \\
\text { PW } \\
(\boldsymbol{\mu s})\end{array}$ & $\begin{array}{c}\text { New Scope } \\
+ \text { PW }(\boldsymbol{\mu s})\end{array}$ & $\begin{array}{c}\text { New Scope } \\
\text {-PW }(\boldsymbol{\mu s})\end{array}$ & $\begin{array}{c}+ \\
\text { \%Error }\end{array}$ & $\begin{array}{c}- \\
\% \text { Error }\end{array}$ \\
\hline $\mathbf{2 0 0}$ & 202 & 196 & $2.00 \%$ & $-1.00 \%$ \\
$\mathbf{4 0 0}$ & 402 & 394 & $1.50 \%$ & $-0.50 \%$ \\
$\mathbf{6 0 0}$ & 602 & 596 & $0.67 \%$ & $-0.33 \%$ \\
$\mathbf{8 0 0}$ & 800 & 792 & $1.00 \%$ & $0.00 \%$ \\
\hline $\mathbf{1 0 0 0}$ & 1000 & 996 & $0.40 \%$ & $0.00 \%$ \\
\hline$* *$
\end{tabular}

Table 4: Positive DAC voltage (Measured vs. Requested)

\begin{tabular}{rrrl}
\hline $\begin{array}{l}\text { GUI } \\
\text { (mA) }\end{array}$ & $\begin{array}{l}\text { Equivalent } \\
\text { Voltage } \\
\text { (V) }\end{array}$ & $\begin{array}{l}\text { New } \\
\text { Voltage } \\
\text { (V) }\end{array}$ & $\begin{array}{l}\text { \%o } \\
\text { Error }\end{array}$ \\
\hline $\mathbf{2}$ & 0.24 & 0.240 & $0.00 \%$ \\
$\mathbf{6}$ & 0.72 & 0.720 & $0.00 \%$ \\
$\mathbf{1 0}$ & 1.2 & 1.200 & $0.00 \%$ \\
$\mathbf{1 4}$ & 1.68 & 1.680 & $0.00 \%$ \\
\hline
\end{tabular}

Table 5: Negative DAC voltage (Measured vs. Requested)

\begin{tabular}{|c|c|c|c|}
\hline $\begin{array}{l}\text { GUI } \\
(\mathrm{mA})\end{array}$ & $\begin{array}{l}\text { Equivalent } \\
\text { Voltage } \\
\text { (V) }\end{array}$ & $\begin{array}{l}\text { New } \\
\text { Voltage } \\
\text { (V) }\end{array}$ & $\begin{array}{l}\% \\
\text { Error }\end{array}$ \\
\hline 2 & 0.24 & 0.240 & $0.00 \%$ \\
\hline 6 & 0.72 & 0.720 & $0.00 \%$ \\
\hline 10 & 1.2 & 1.200 & $0.00 \%$ \\
\hline 14 & 1.68 & 1.680 & $0.00 \%$ \\
\hline
\end{tabular}

$* *$ Note: The equivalent voltage was calculated by using the ideal $\mathrm{R}=120 \Omega$. ** 


\section{Saline Experiment}

A recording oscilloscope with two differential probes (see Appendix F: Equipment for equipment details) was used to record all measurements. Matlab was used to apply the sense filter and plot the ICD waveforms after the sense filter was applied. Excel was used to plot the waveforms before the sense filter was applied. The results are summarized in Table 6 . The pre and post sense filter waveforms are located in section Saline Tank Waveforms in Appendix A: Pulse Generator Waveforms.

Table 6: Maximum recorded negative and positive amplitudes for all 4 waveforms at $200 \mu$ s and $500 \mu s$ pulse widths

\begin{tabular}{|l|l|l|l|l|l|l|l|l|}
\hline PW & \multicolumn{3}{|c|}{ Negative Max Amplitude( $\boldsymbol{\mu V})$} & \multicolumn{3}{c|}{ Positive Max Amplitude $(\boldsymbol{\mu V})$} \\
$\mathbf{( \mu \mathbf { \mu } )}$ & Monophasic & Biphasic & Triphasic & Pentapolar & Monophasic & Biphasic & Triphasic & Pentapolar \\
\hline $\mathbf{2 0 0}$ & 795.7 & 133.1 & 38.4 & 46.2 & 870.6 & 117.1 & 31.7 & 24.6 \\
\hline $\mathbf{5 0 0}$ & 975.5 & 181.3 & 83.8 & 73.8 & 1072.0 & 200.9 & 87.2 & 90.8 \\
\hline
\end{tabular}

\section{Discussions and Conclusions}

The analytical results in Figure 15, Figure 16, and Figure 17 showed that the triphasic pulse type has the smallest magnitude of all the other pulse types in the $1-250 \mathrm{~Hz}$ region. It showed a significantly lower magnitude when compared to the monophasic and biphasic pulse types, the current pulse types used in SCS devices today. Looking at Figure 17, the triphasic pulse did begin to have a slightly higher magnitude than the biphasic pulse at frequencies around $250 \mathrm{~Hz}$. Since the intrinsic cardiac events that the ICD is trying to sense typically have frequencies $<120 \mathrm{~Hz}$, the magnitudes around $250 \mathrm{~Hz}$ should have minimal effect on cross-talk.

Figure 19 and Figure 20 show that the magnitude response at an interphasic gap of $1 \mu \mathrm{s}$ has minimal effect on the magnitude response of the triphasic waveform when compared to an interphasic gap of 0 . This provides proof that the programmed $1 \mu$ s interphasic delay in the pulse generator does not significantly affect the results. These figures also show that a large enough interphasic gap will affect the results and provide a greater magnitude response.

One difference between the ideal pulse waveforms used in the analytical experiment and the actual pulse generator waveforms is how the pulse width was configured. Each phase of the ideal waveforms have the same pulse width, where the pulse generator waveforms are configured to use the same amount of energy per pulse. For example: The pulse generator with a programmed pulse width of $200 \mu$ s will output a monophasic pulse with a width of $400 \mu \mathrm{s}$, a biphasic pulse with two phases of $200 \mu \mathrm{s}$ each, a triphasic pulse with 3 phases of $100 \mu \mathrm{s}, 200 \mu \mathrm{s}$, 
and $100 \mu \mathrm{s}$, and a pentapolar pulse with 5 phases of $100 \mu \mathrm{s}, 100 \mu \mathrm{s}, 100 \mu \mathrm{s}$ (off), $100 \mu \mathrm{s}$, and $100 \mu \mathrm{s}$. The results between the analytical and in vitro experiments are still comparable because the analytical equations are normalized to 1 so the energy of the pulse should not matter. A good experiment to prove that assumption would be to generate new equations that match the pulse generator waveform strategy and redo the analytical experiment.

Table 2, Table 3, Table 4, and Table 5 show that the programmable settings on the GUI are very close to the actual pulse output by the pulse generator. Since actual instruments were used during this verification, there are sources of error. The oscilloscope has maximum resolution (Tektronix, 2012) which directly impacts the pulse period and pulse width measurements; the pulse width measurements are the most affected because they are much smaller than the pulse period. The SMU is much more accurate than the oscilloscope, which is one reason why the \%error in Table 4 and Table 5 are all 0 . The source of error in the amplitude measurement will not show up with this method because it is due to the variability of the resistors used in the "V-I Pulse Converter" circuit; the $120 \Omega$ sense resistor will have the most impact. The amplitude verification method only verified the voltage coming out of the two DACs and compared them to the programed equivalent voltage using an ideal sense resistor value of $120 \Omega$. The sense resistor has a tolerance of $1 \%$ so the error in this measurement will be bound by $1 \%$ plus the inaccuracy of the SMU (Keithley, 2013). The most direct method would have been to use a precision sense resistor at the output of the pulse generator and measured the voltage across that resistor. The current could then be calculated using "Ohms Law". However, this method would need to use an oscilloscope which has its own inaccuracies.

The in vitro testing results summarized in Table 6 show that the cross-talk between an SCS and an ICD device are significantly reduced when using a triphasic pulse compared to a biphasic and monophasic pulse. The cross-talk amplitude was reduced by $>3.4 \mathrm{x}$ and $>20 \mathrm{x}$ at a pulse width of $200 \mu$ s when compared to a monophasic and biphasic pulse. The cross-talk was also reduced by $>2.1 \mathrm{x}$ and $>11 \mathrm{x}$ at a pulse width of $500 \mu \mathrm{s}$. The cross-talk was comparable between the pentapolar and triphasic pulses at both 200 and $500 \mu$ s pulse widths. The difference between the pentapolar and triphasic pulses may be smaller than the resolution of the oscilloscope that was used to measure the waveforms. The analytical data between a pentapolar and triphasic pulse also showed a similar magnitude response so similar cross-talk amplitudes between the two pulses were expected.

Looking at the post sense filter waveforms in Figure 34, Figure 35, Figure 40, and Figure 41 , the duration of the cross-talk is also minimized when using a triphasic pulse vs. a monophasic and biphasic pulse. This is significant because the longer the cross-talk is present, the greater the probability a SCS pulse will fall in the sensing window and not in the blanking or 
refractory periods. The results from this saline experiment between the triphasic and pentapolar pulses are inconclusive but either pulse will drastically improve the cross-talk compared to the monophasic and biphasic pulses which are used currently in the SCS devices.

Both pulses, a triphasic or pentapolar, could be used in the SCS devices to improve crosstalk with patients who have both an ICD and SCS device implanted. One avenue that this research did not investigate is how the stimulation threshold varies between the different pulse types. For example: What if a patient needs $2 \mathrm{x}$ the pulse amplitude with a triphasic pulse vs. a biphasic pulse to get the same stimulation? The cross-talk will not be improved in that patient because you have increased the amplitude by $2 x$ and the triphasic pulse only decreases the crosstalk by $\sim 2 \mathrm{x}$ at $500 \mu \mathrm{s}$. In this scenario, the increase in amplitude counteracts the cross-talk improvements. To continue analyzing the benefits of the triphasic pulse, the stimulation threshold between the pulses, especially biphasic and triphasic, should be analyzed and compared. In any case, the results of the work described here prove that cross-talk between an SCS and ICD device implanted in the same patient are significantly decreased when using a triphasic pulse compared to the current pulse types used in SCS device today. 


\section{Bibliography}

Andersen, C., Oxhoj, H., \& Arnsbo, P. (1990). Management of Spinal Cord Stimulators in Patients with Cardiac Pacemaker. PACE.

Bernstein, S. A., Wong, B., Vasquez, C., Rosenberg, S., Rooke, R., Kuznekoff, L. M., et al. (2012). Spinal Cord Stimulation Protects Against Atrial Fibrillation Induced by Tachypacing. Heart Rhythm, 1426-1433.

Bharmi, R., Bornzin, G. A., \& Poore, J. W. (2010). Patent No. 20100331921. United States of America.

Dartmouth-Hitchcock. (2013). Spinal Cord Stimulation. Retrieved 4 23, 2013, from DartmouthHitchcock: http://patients.dartmouthhitchcock.org/pain_mgt/spinal_cord_stimulation.html

Dejongste, M. J., Terhorst, G. J., \& Foreman, R. D. (2009). Basic Research Models for the Study of Underlying Mechanisms of Electrical Neuromodulation and Ischemic Heart-Brain Interactions. Cleveland Clinic Journal of Medicine.

Franco, S. (2007). Design with Operational Amplifiers and Analog Integrated Circuits. New Delhi: Tata McGraw-Hill Publishing Company Limited.

Geddes, L. A., \& Baker, L. E. (1989). Principles of Applied Biomedical Instrumentation (3rd Edition). New York: John Wiley \& Sons.

Keithley. (2013). 2400 Broad Pulse SourceMeter. Retrieved 4 20, 2013, from Keithley: http://www.keithley.com/products/dcac/voltagesource/broadpurpose/?mn=2400

Koeppen, B. M., \& Stanton, A. B. (2008). Berne \& Levy Physiology (Vol. Sixth Edition). Elsevier, Inc.

MattairTech. (2012, 7 27). MT-X1 Manual. Retrieved 10 1, 2012, from MattairTech: http://www.mattairtech.com/docs/MT-X1/MT-X1_Manual.pdf

Medtronic CRMD. (2013, 4 23). Determining the Feasibility of Spinal Cord Neuromodulation for the Treatment of Chronic Heart Failure. Retrieved 5 22, 2013, from ClinicalTrials.gov: http://www.clinicaltrials.gov/ct2/results?term=DEFEATHF\&Search $=$ Search

Molon, G., Perrone, C., Maines, M., Costa, A., Comisso, J., Boi, A., et al. (2011). ICD and Neuromodulation Devices: Is Peaceful Coexistence Possible? PACE, 690-693.

Moore, W. J. (1972). Physical Chemistry (4th Edition). New Jersey: Prentice-Hall. 
Moses, H. W., Miller, B. D., Moulton, K. P., \& Schneider, J. A. (2000). A Practical Guide to Cardiac Pacing (5th ed.). Philadelphia: Lippincott Williams \& WIlkins.

Mozzi, L., Neviani, A., \& Baschirotto, A. (2001). A 1uA Front-End for Pacemaker Atrial Sensing Channels. IMEC.

Poore, J. W. (2012, 9 26). Principal Staff Scientist. (R. Wensley, Interviewer)

Ridder, D. D., Plazier, M., Kamerling, N., Menovsky, T., \& Vanneste, S. (2013). Burst Spinal Cord Stimulation for Limb and Back Pain. World Neurosurgery.

Shealy, C. N., Mortimer, J. T., \& Reswick, J. B. (1967). Electrical Inhibition of Pain by Stimulation of the Dorsal Columns. Boston.

St. Jude Medical. (2012). Quadra Assura CRT-D. Retrieved 4 20, 2013, from St. Jude Medical: http://www.sjmprofessional.com/Products/US/CRT-Systems/Quadra-Assura-CRTD.aspx

St. Jude Medical. (2013, 4 1). Spinal Cord Stimulation for Heart Failure. Retrieved 5 22, 2013, from ClinicalTrials.gov: http://www.clinicaltrials.gov/ct2/show/NCT01362725?term=SCS-HEART\&rank=1

Stojanovic, M. P., \& Abdi, S. (2002). Spinal Cord Stimulation. Pain Physician, 156-166.

Taylor, R. S., Vries, J. D., Buchser, E., \& DeJongste, M. J. (2009). Spinal Cord Stimulation in the Treatment of Refractory Angina: Systematic Review and Meta-Analysis of Randomised Controlled Trials. BMC Cardiovascular Disorders.

Tektronix. (2012). User Manual TDS3000 Series Digital Phosphor Oscilloscopes. Retrieved 4 20, 2013, from Washington.edu:

http://www.cs.washington.edu/education/courses/cse466/07wi/labs/12/Oscope/TDS3000 Manual.pdf

Thammanomai, A., Sweeney, M. O., \& Eisenberg, S. R. (2006). A Comparison of the Output Characteristics of Several Implantable Cardioverter-Defibrillators. Heart Rhythm, 10531059.

Tidy, C., \& Rull, G. (2010, 5 21). Pacemakers and Pacing. Retrieved 3 9, 2013, from Patient.co.uk: http://www.patient.co.uk/doctor/Pacemakers-and-Pacing.htm

Vaseghi, M., \& Shivkumar, K. (2012). Neuraxial Modulation for Ventricular Arrhythmias: A New Hope. Heart Rhythm, 1884-1887.

Wall, P. D., \& Melzack, R. (1965). Pain Mechanisms: A New Theory. Science, 971-9. 


\section{Appendix A: Pulse Generator Waveforms}

\section{A. Theoretical Waveforms}

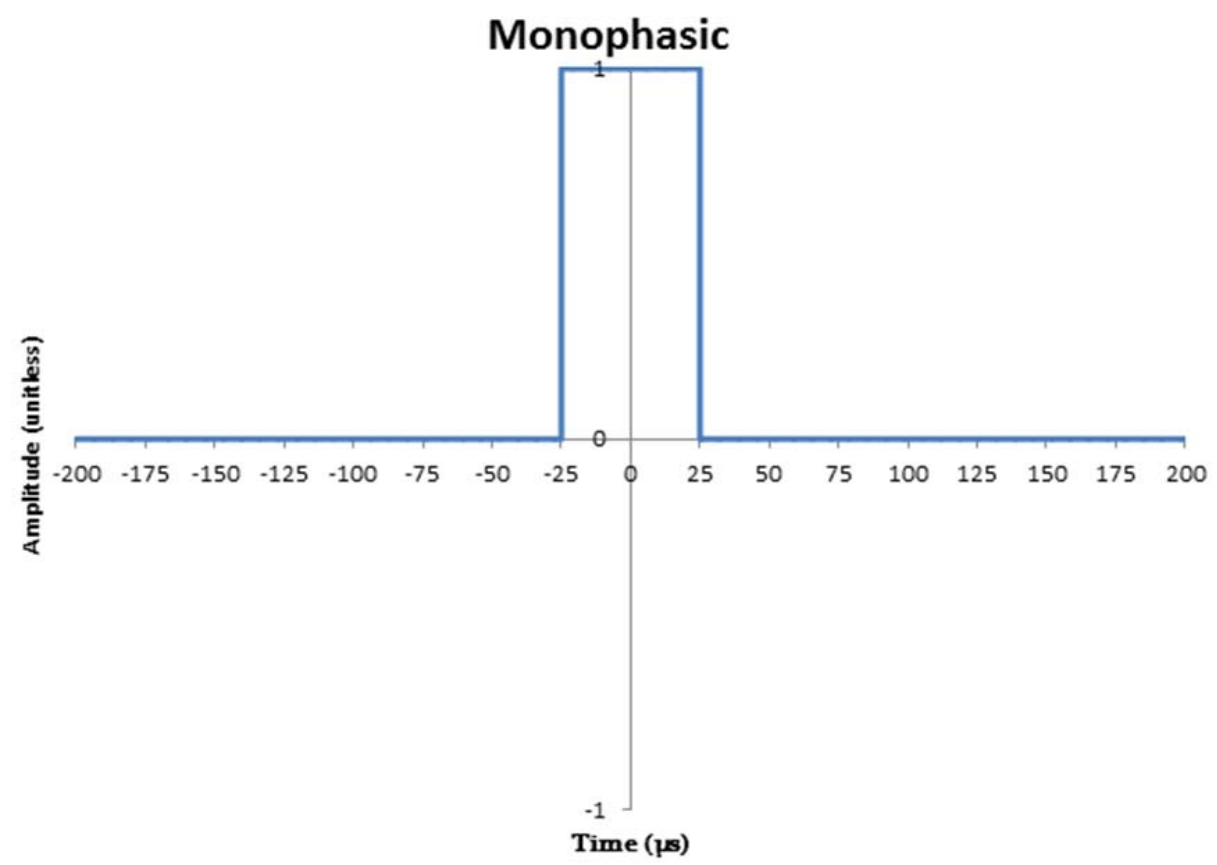

Figure 21: Ideal monophasic waveform with $50 \mu$ s pulse width

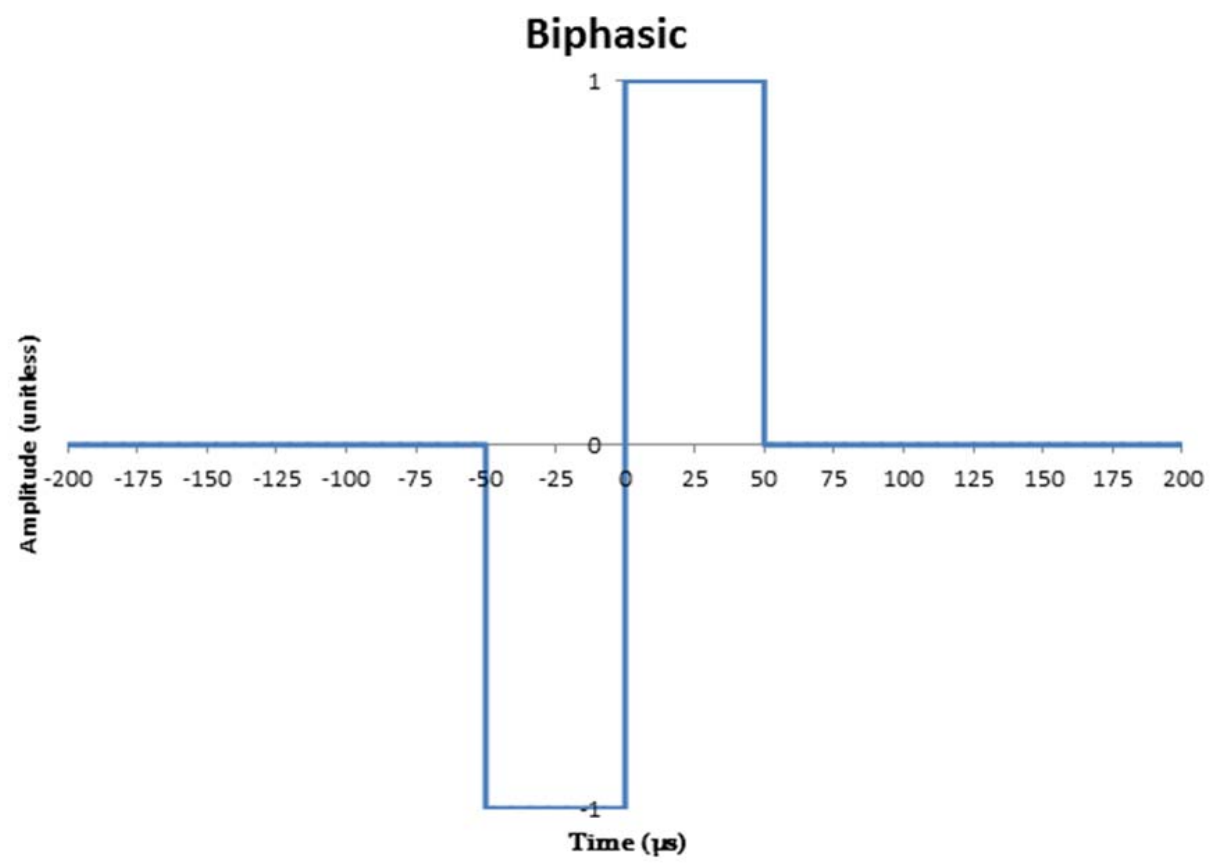

Figure 22: Ideal biphasic waveform with $50 \mu$ sulse width 


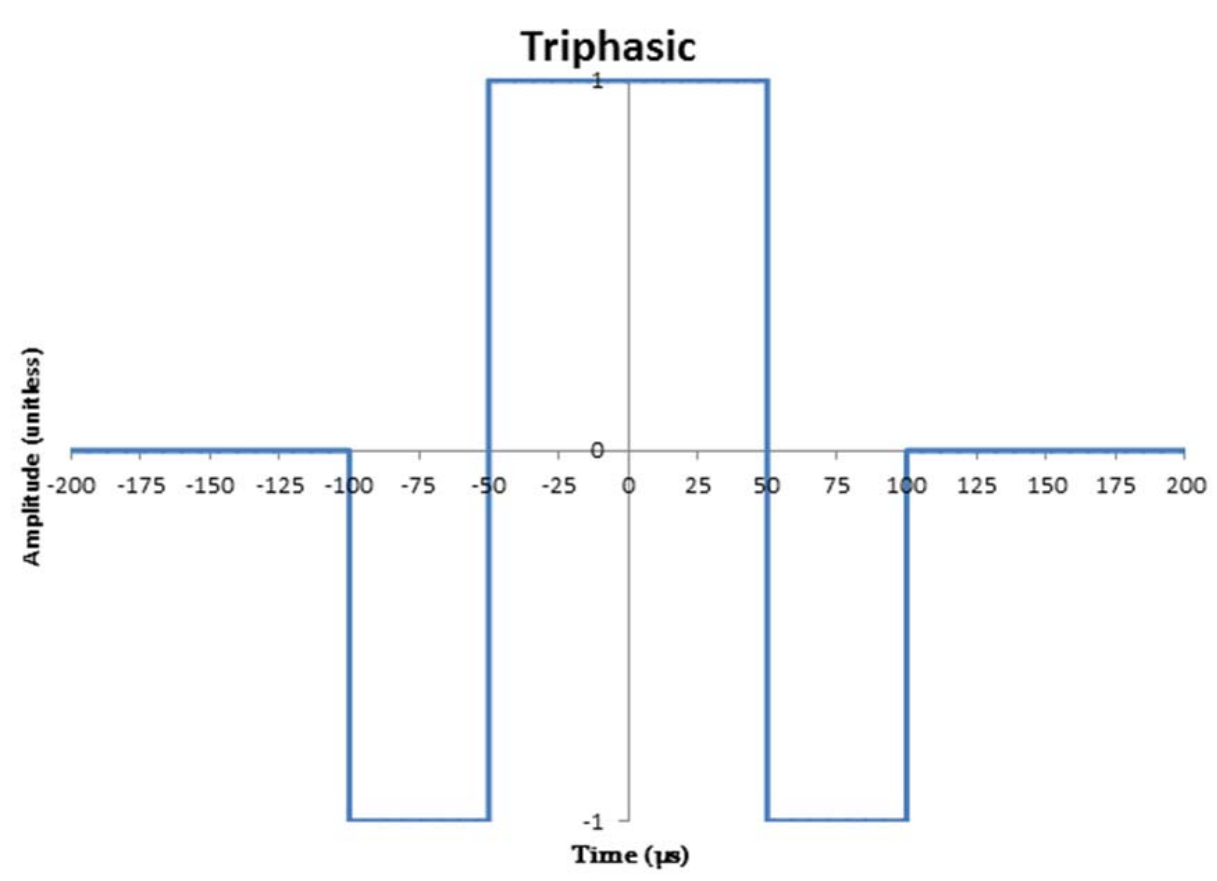

Figure 23: Ideal triphasic waveform with $50 \mu$ s pulse width

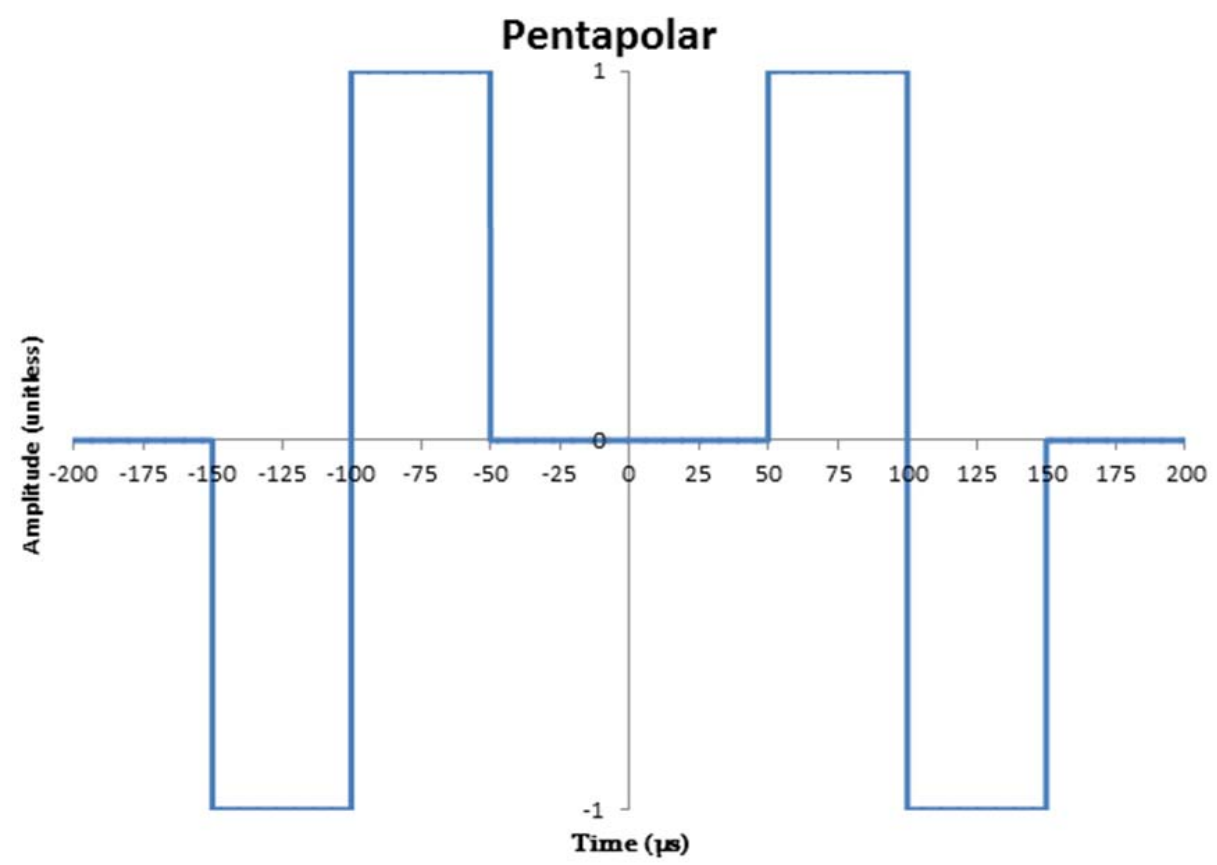

Figure 24: Ideal pentapolar waveform with $50 \mu$ s pulse width 


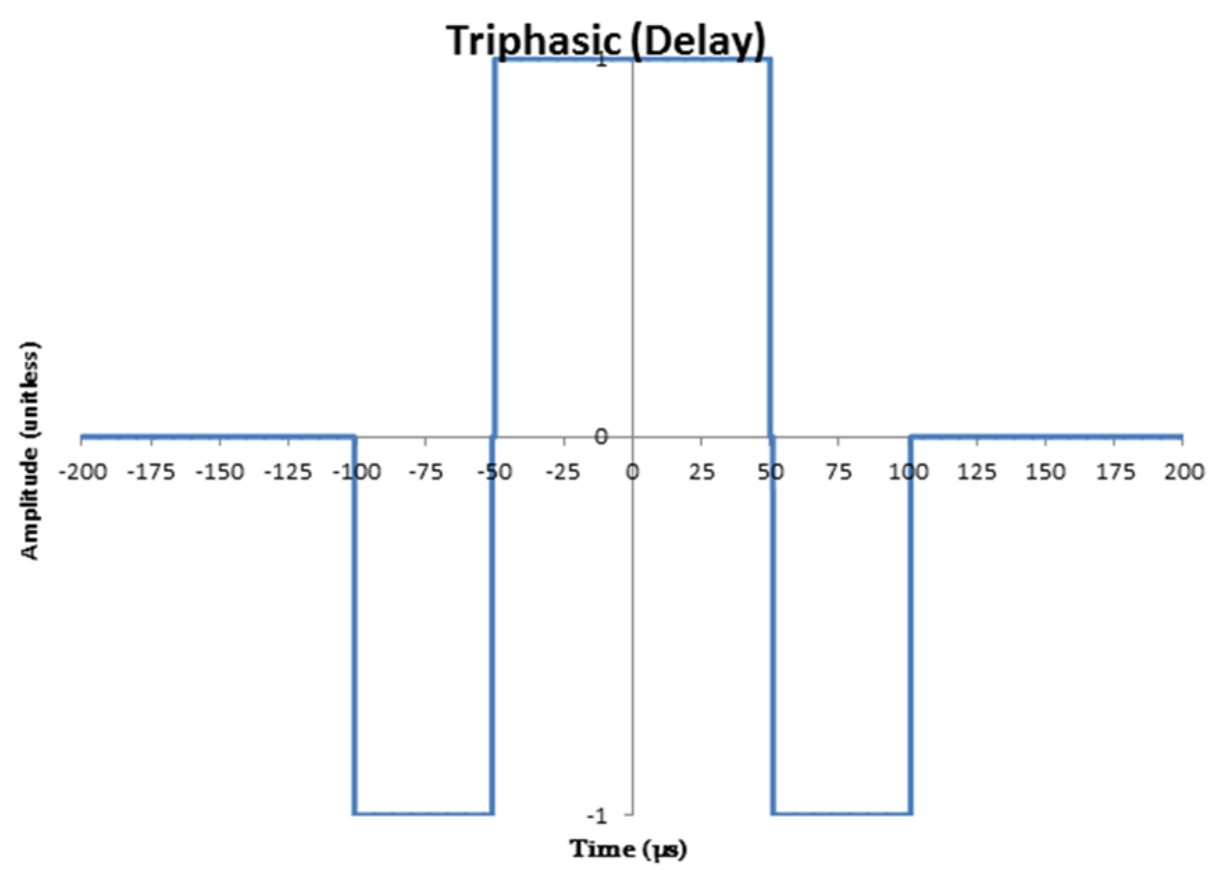

Figure 25: Ideal triphasic waveform with $50 \mu$ s pulse width and a $1 \mu$ s interphasic delay

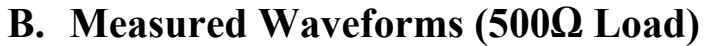

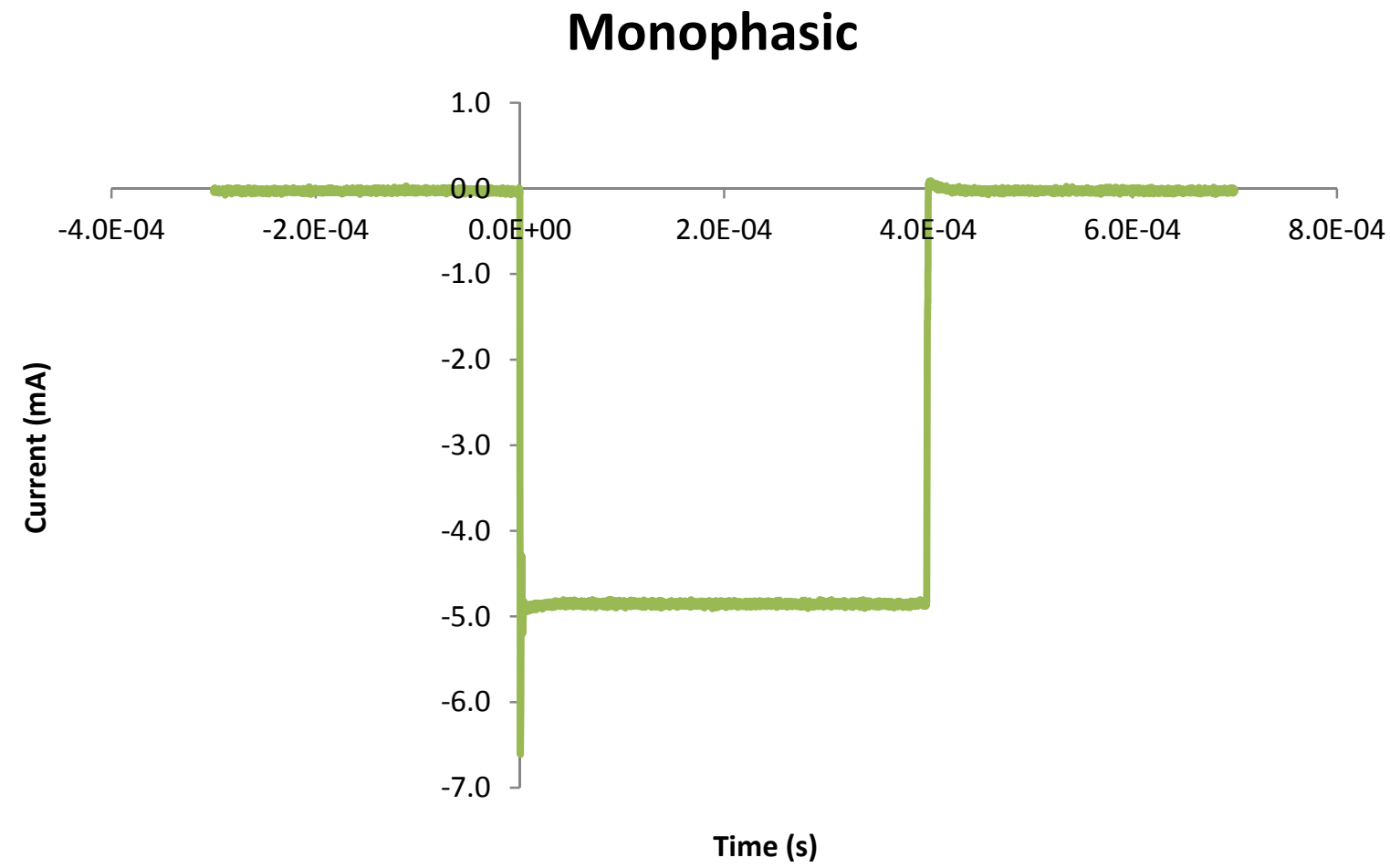

Figure 26: Monophasic waveform $(\mathrm{PW}=200 \mu \mathrm{s}$, Amplitude $=5 \mathrm{~mA})$ 


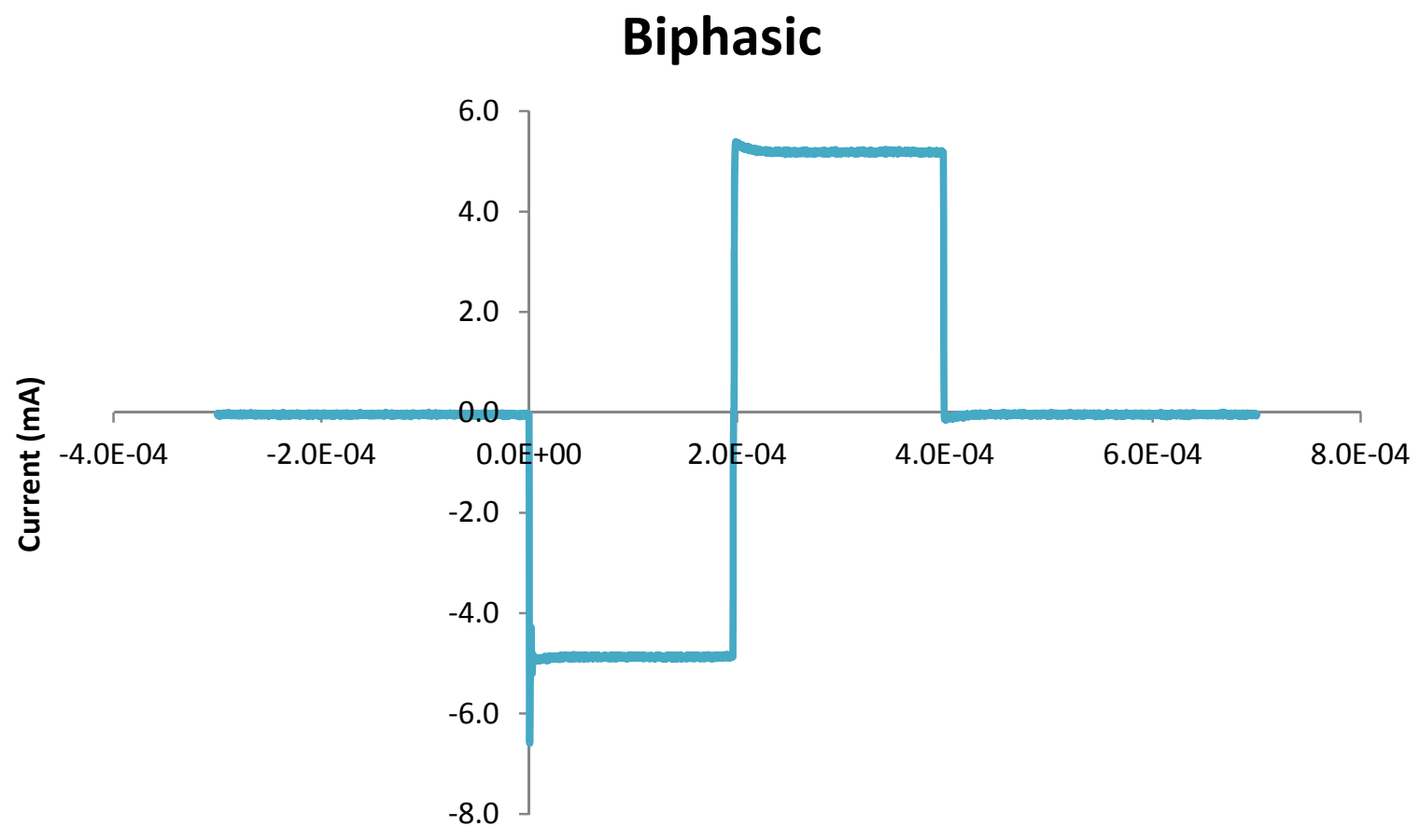

Time (s)

Figure 27: Biphasic waveform $(\mathrm{PW}=200 \mu \mathrm{s}$, Amplitude $=5 \mathrm{~mA})$

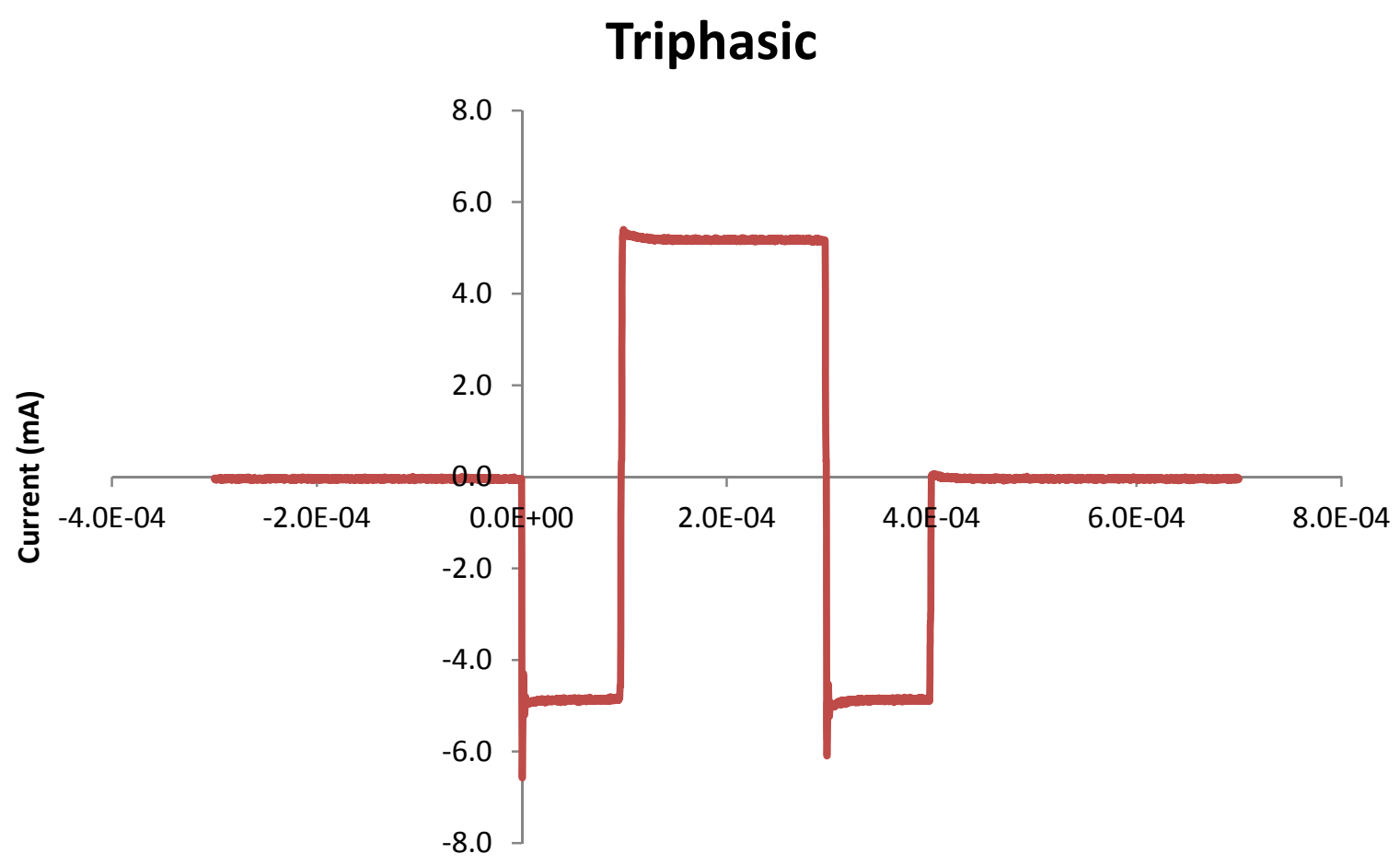

Time (s)

Figure 28: Triphasic waveform $(\mathrm{PW}=200 \mu \mathrm{s}$, Amplitude $=5 \mathrm{~mA})$ 


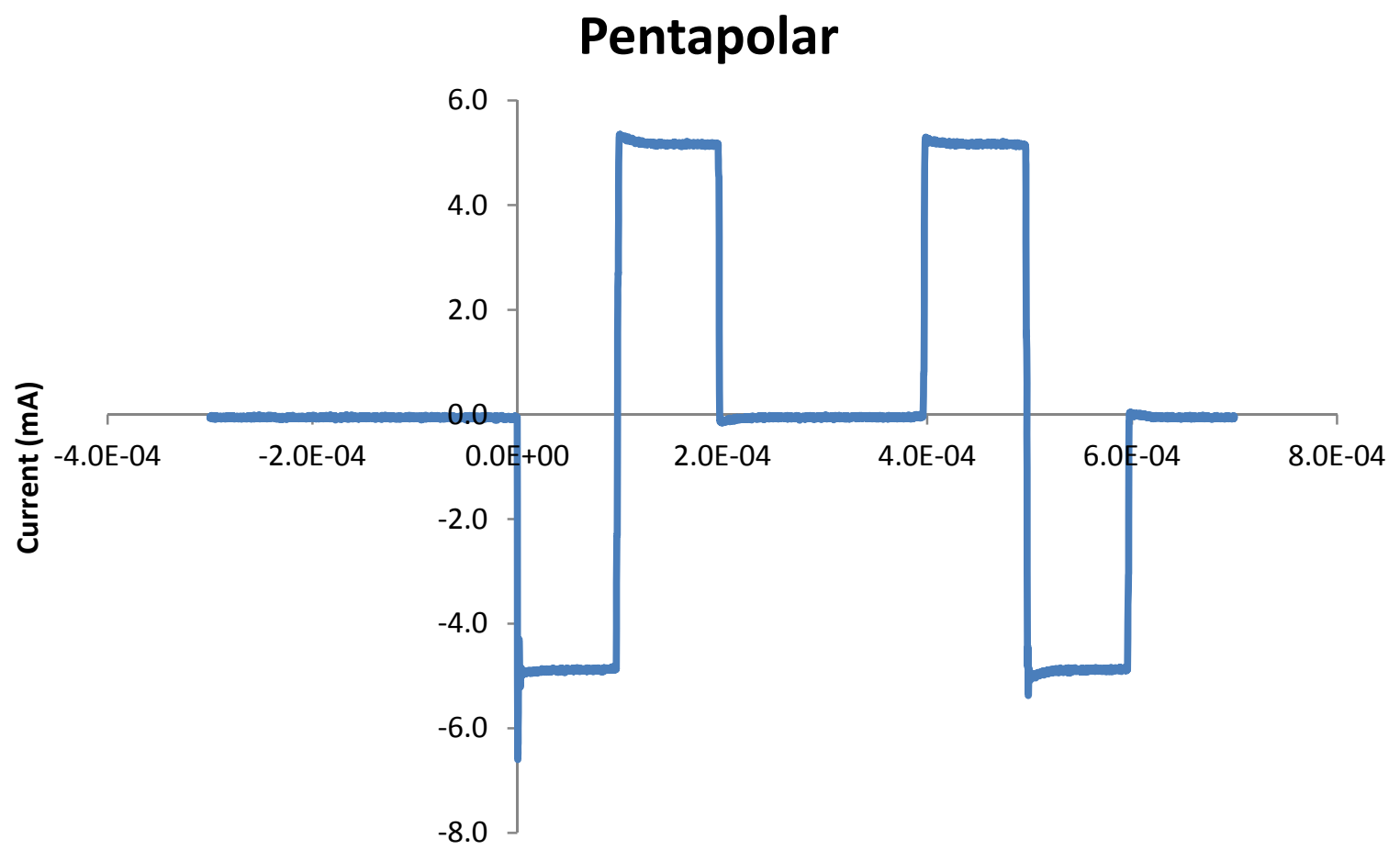

Time (s)

Figure 29: Pentapolar waveform $(\mathrm{PW}=200 \mu \mathrm{s}$, Amplitude $=5 \mathrm{~mA})$ 


\section{Saline Tank Waveforms}

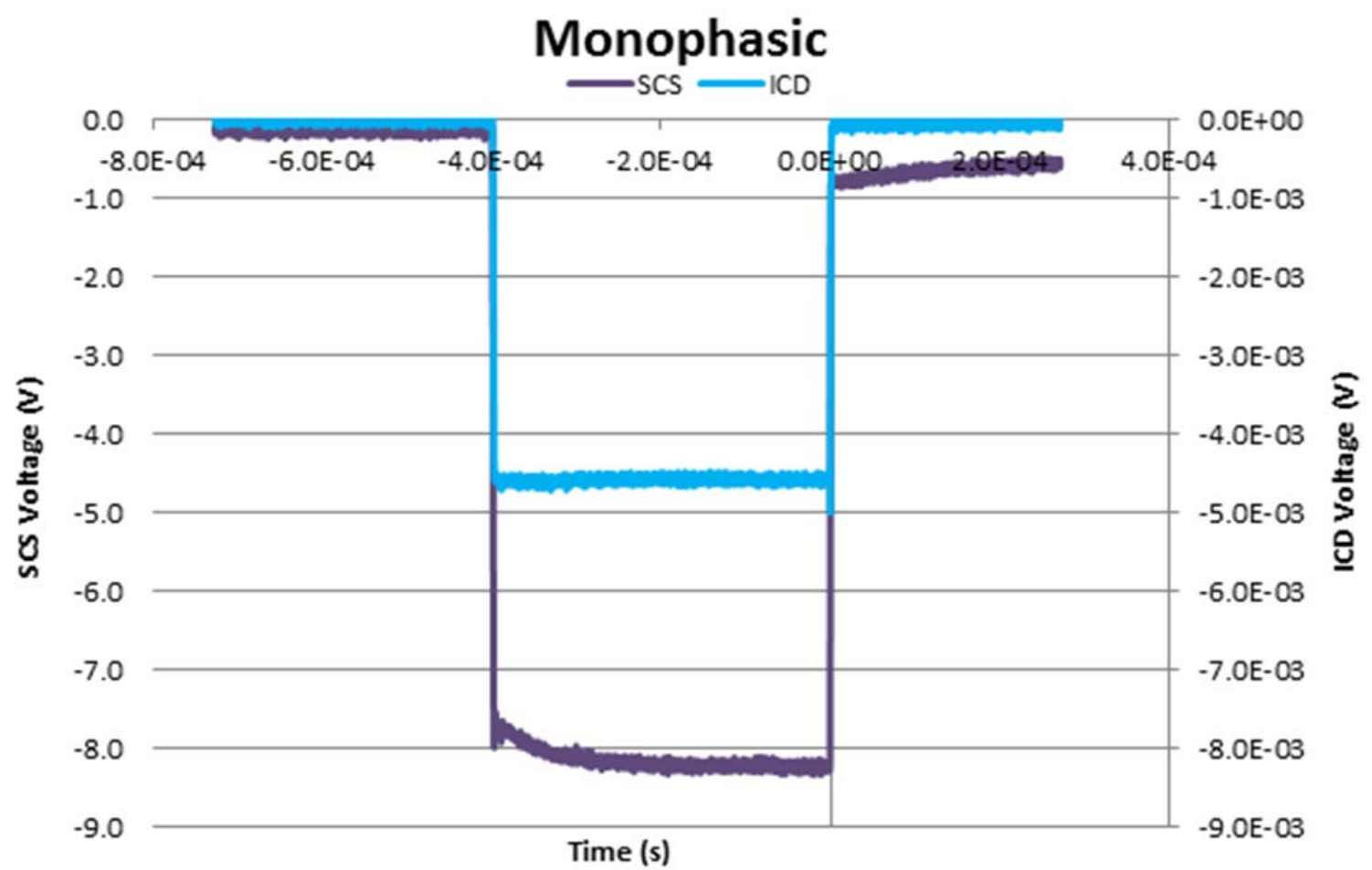

Figure 30: Monophasic ICD and SCS waveforms before the sense filter is applied $(\mathrm{PW}=200 \mu \mathrm{s})$

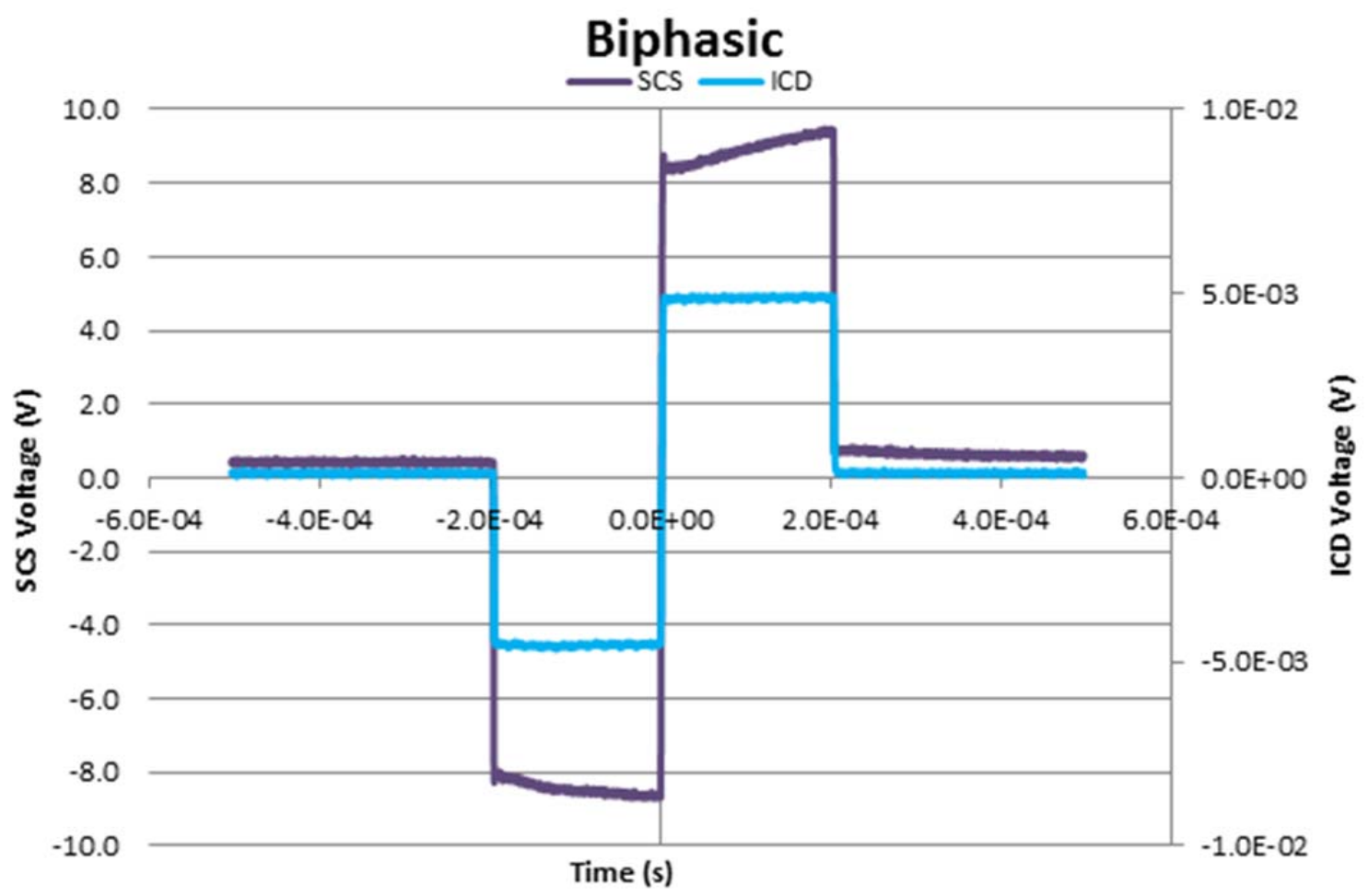

Figure 31: Biphasic ICD and SCS waveforms before the sense filter is applied $(P W=200 \mu s)$ 


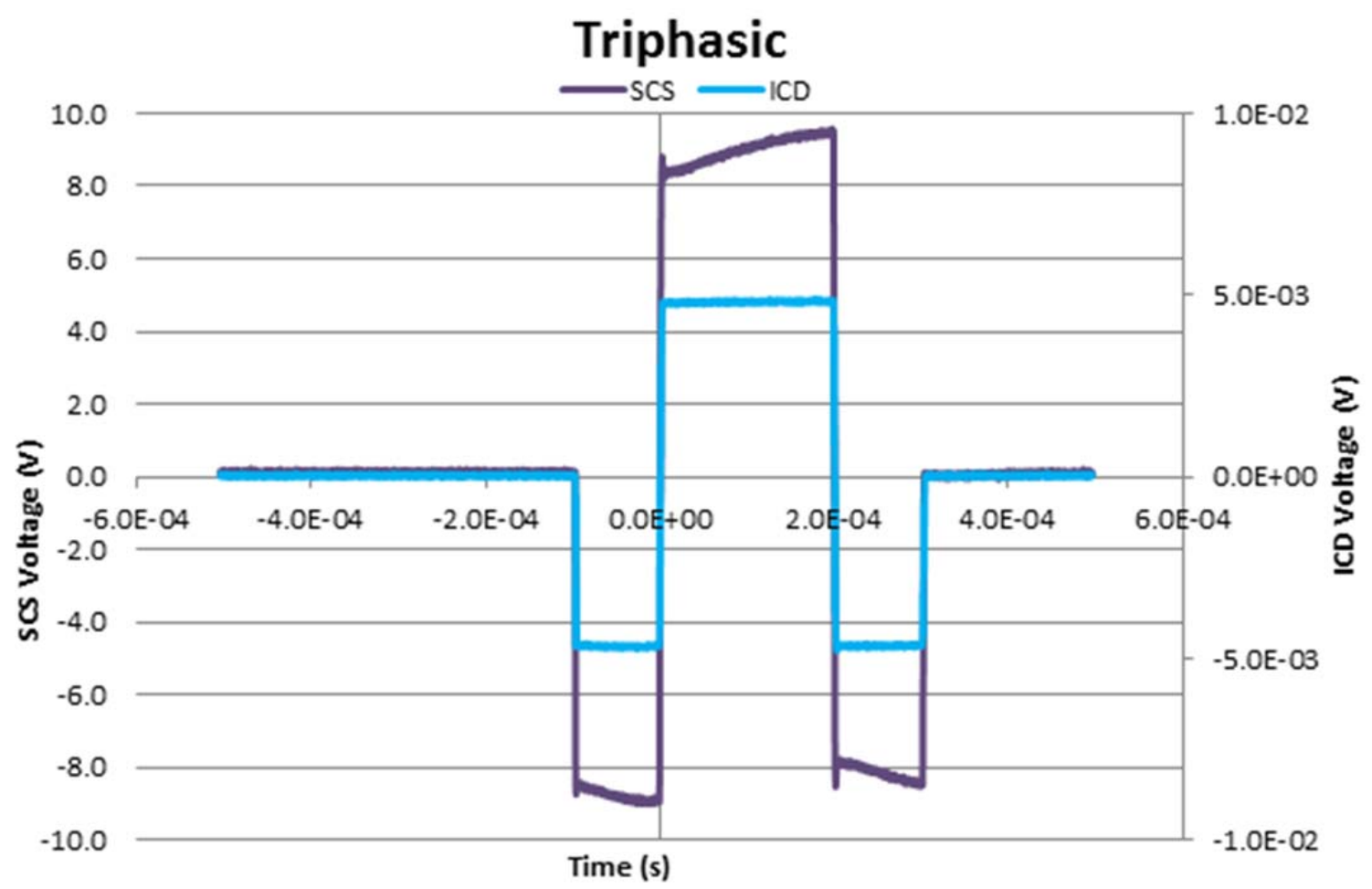

Figure 32: Triphasic ICD and SCS waveforms before the sense filter is applied ( $\mathrm{PW}=200 \mu \mathrm{s})$

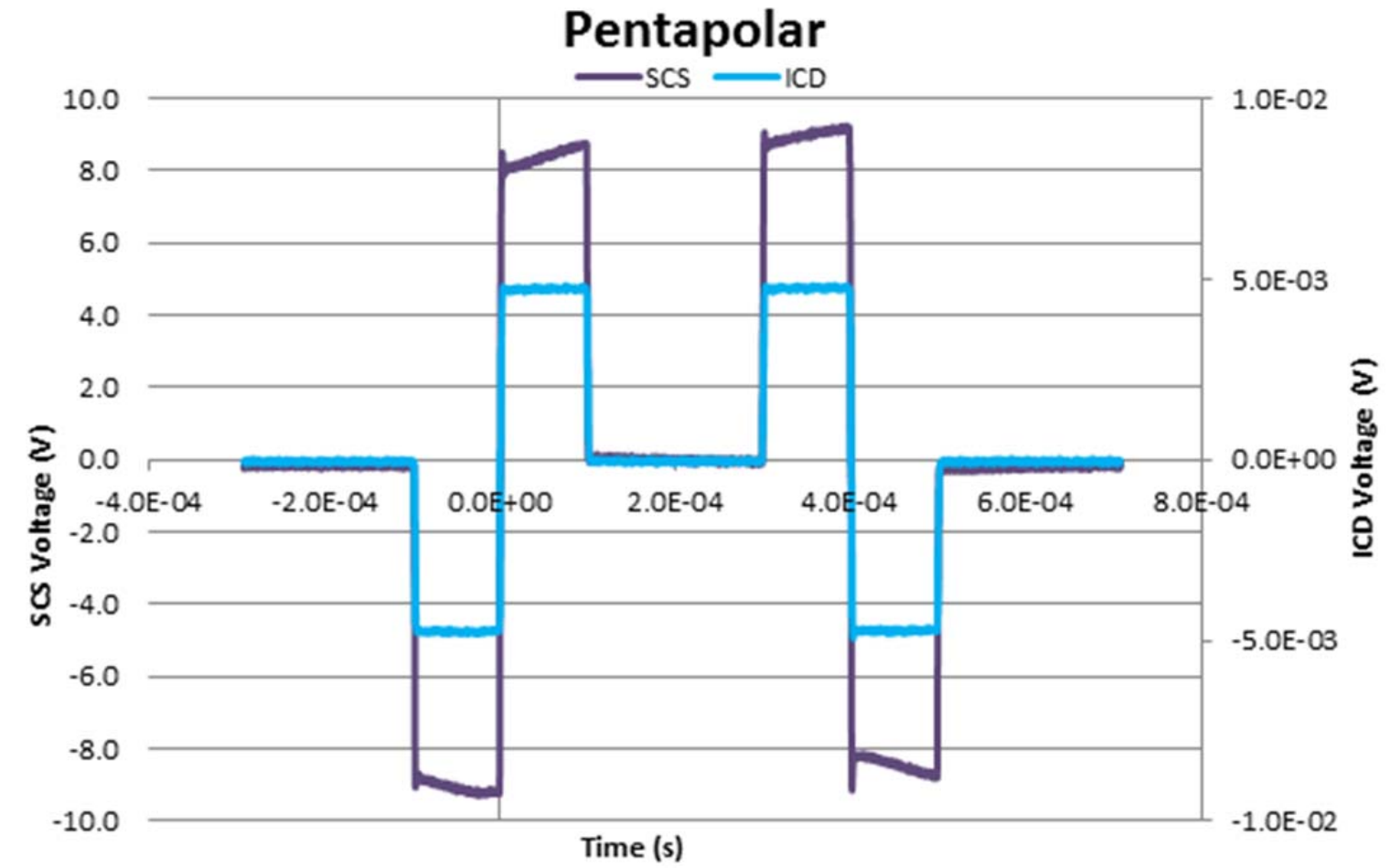

Figure 33: Pentapolar ICD and SCS waveforms before the sense filter is applied (PW $=200 \mu s)$ 


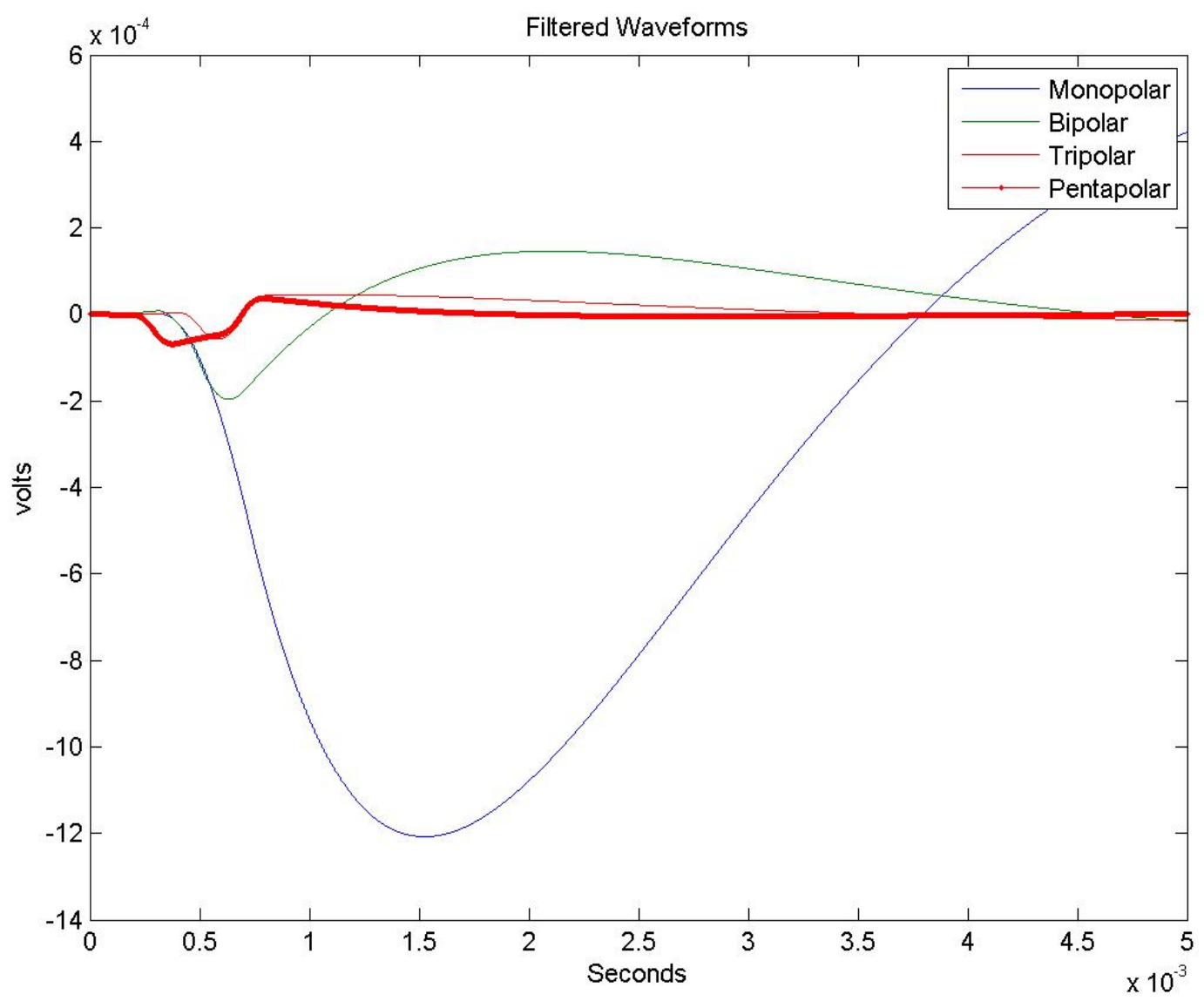

Figure 34: All 4 ICD waveforms post sense filter with a programmed pulse width $=200 \mu \mathrm{s}$ 


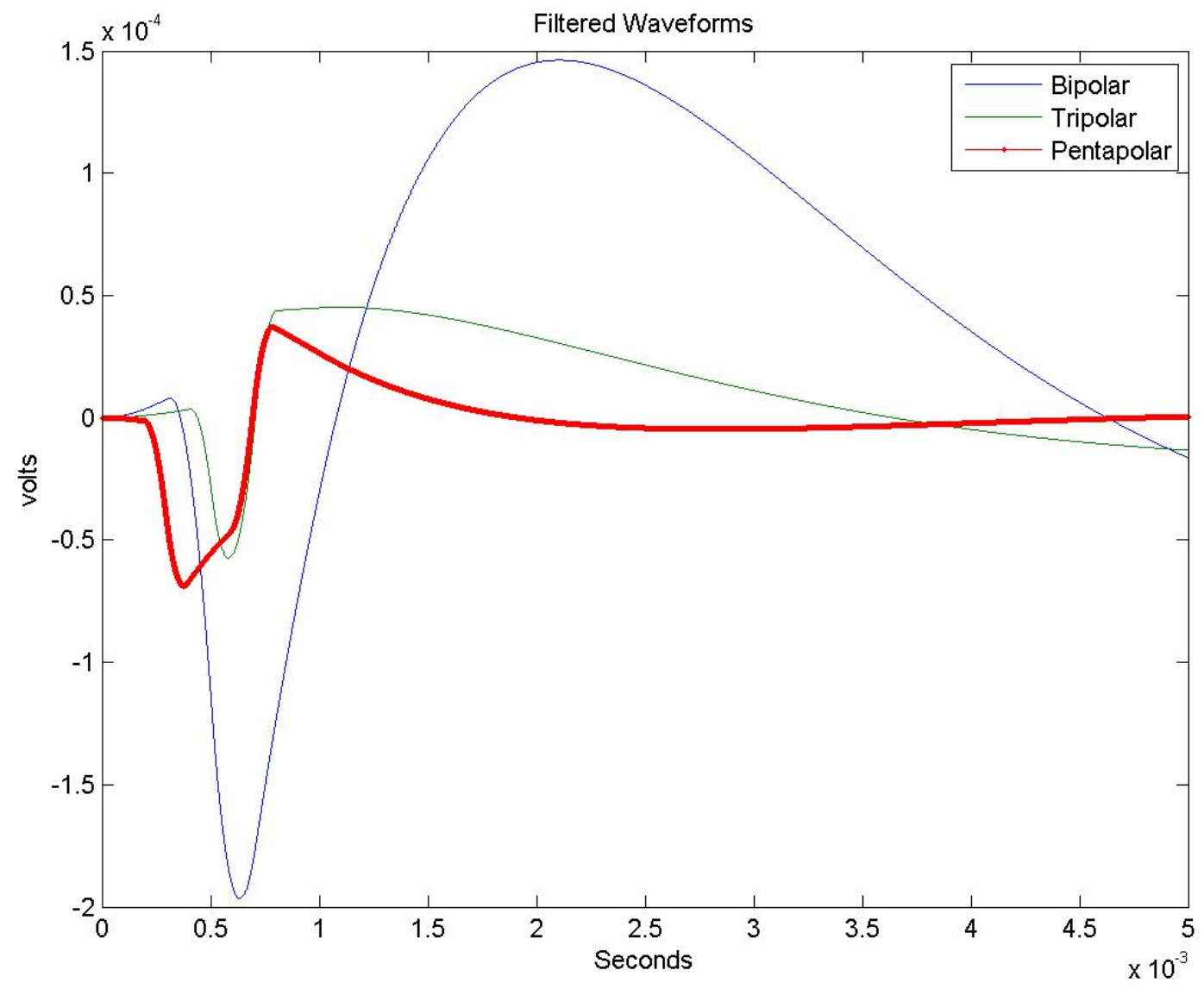

Figure 35: Biphasic, Triphasic, and Pentapolar ICD waveforms post sense filter with a programmed pulse width $=200 \mu \mathrm{s}$ 


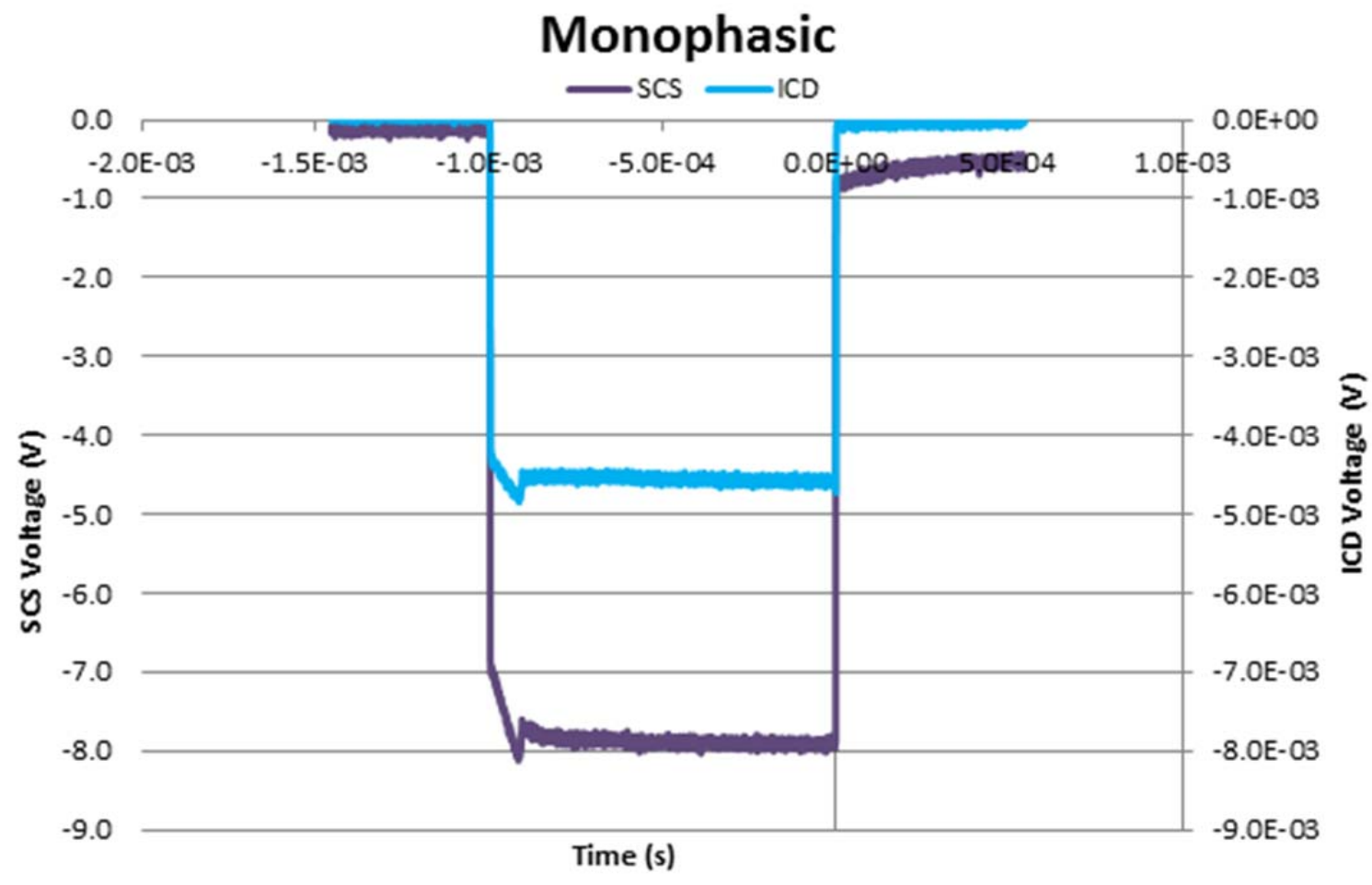

Figure 36: Monophasic ICD and SCS waveforms before the sense filter is applied (PW $=500 \mu s)$

\section{Biphasic}

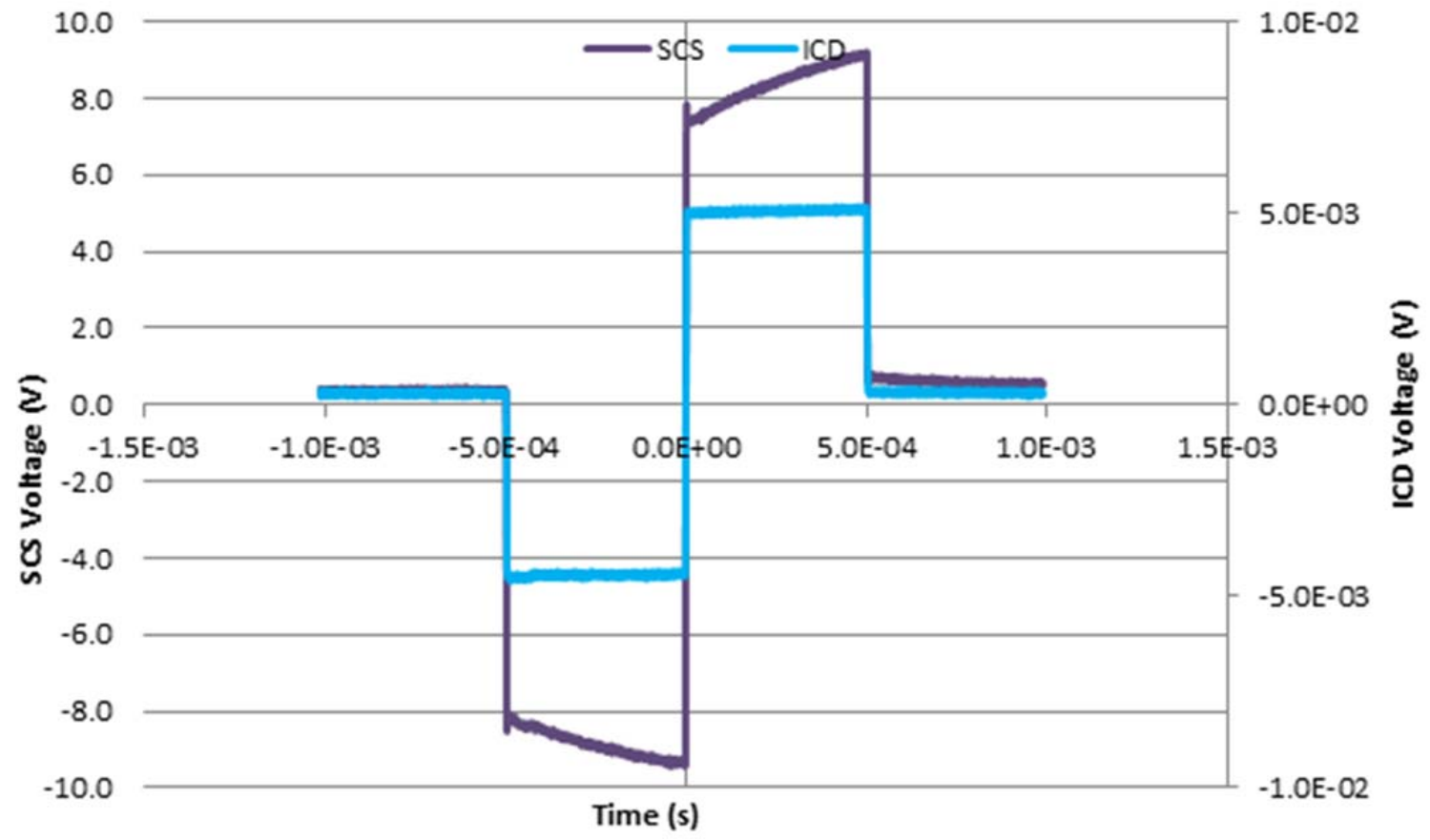

Figure 37: Biphasic ICD and SCS waveforms before the sense filter is applied $(\mathrm{PW}=500 \mu s)$ 


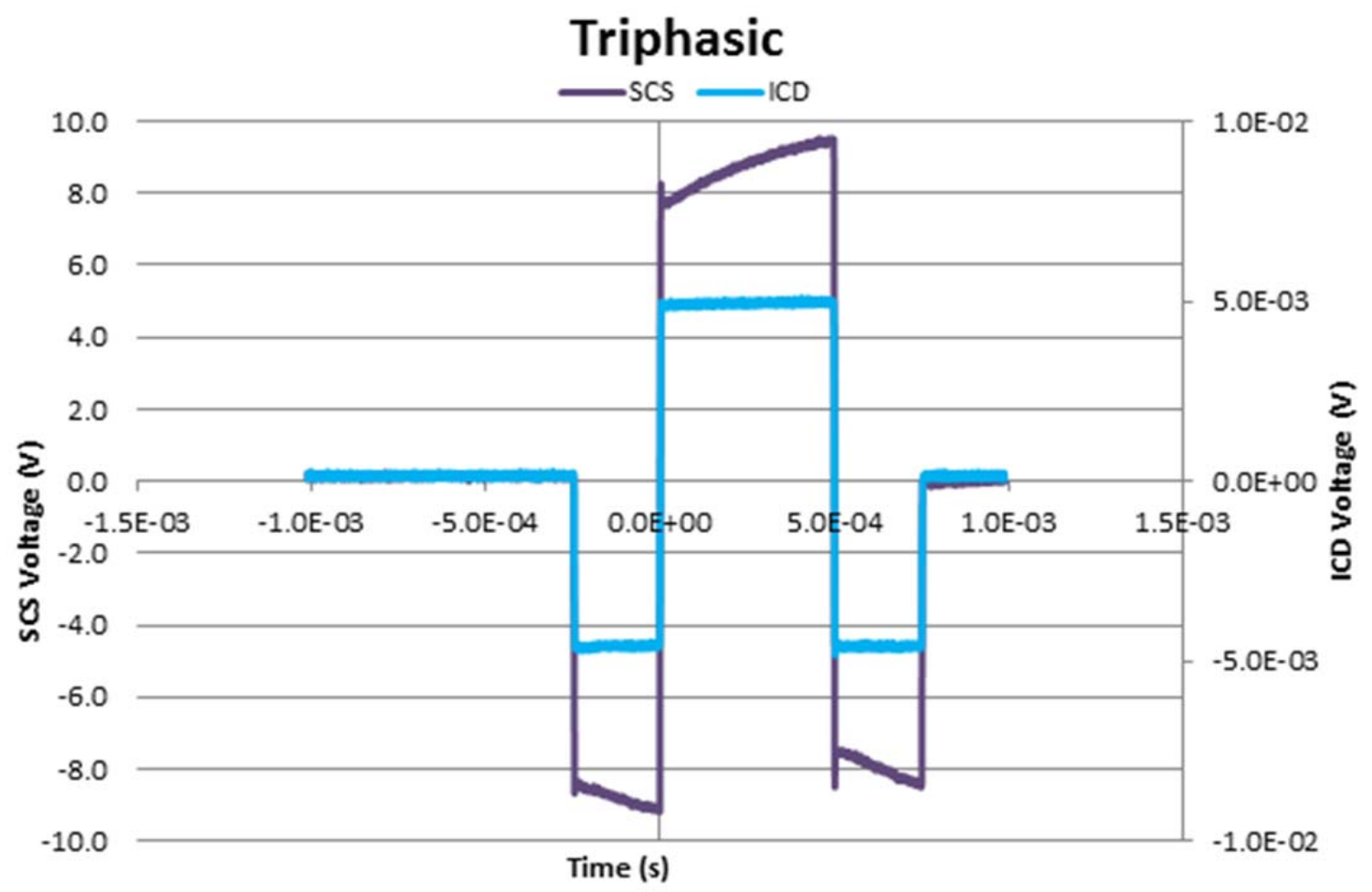

Figure 38: Triphasic ICD and SCS waveforms before the sense filter is applied ( $\mathrm{PW}=500 \mu \mathrm{s})$

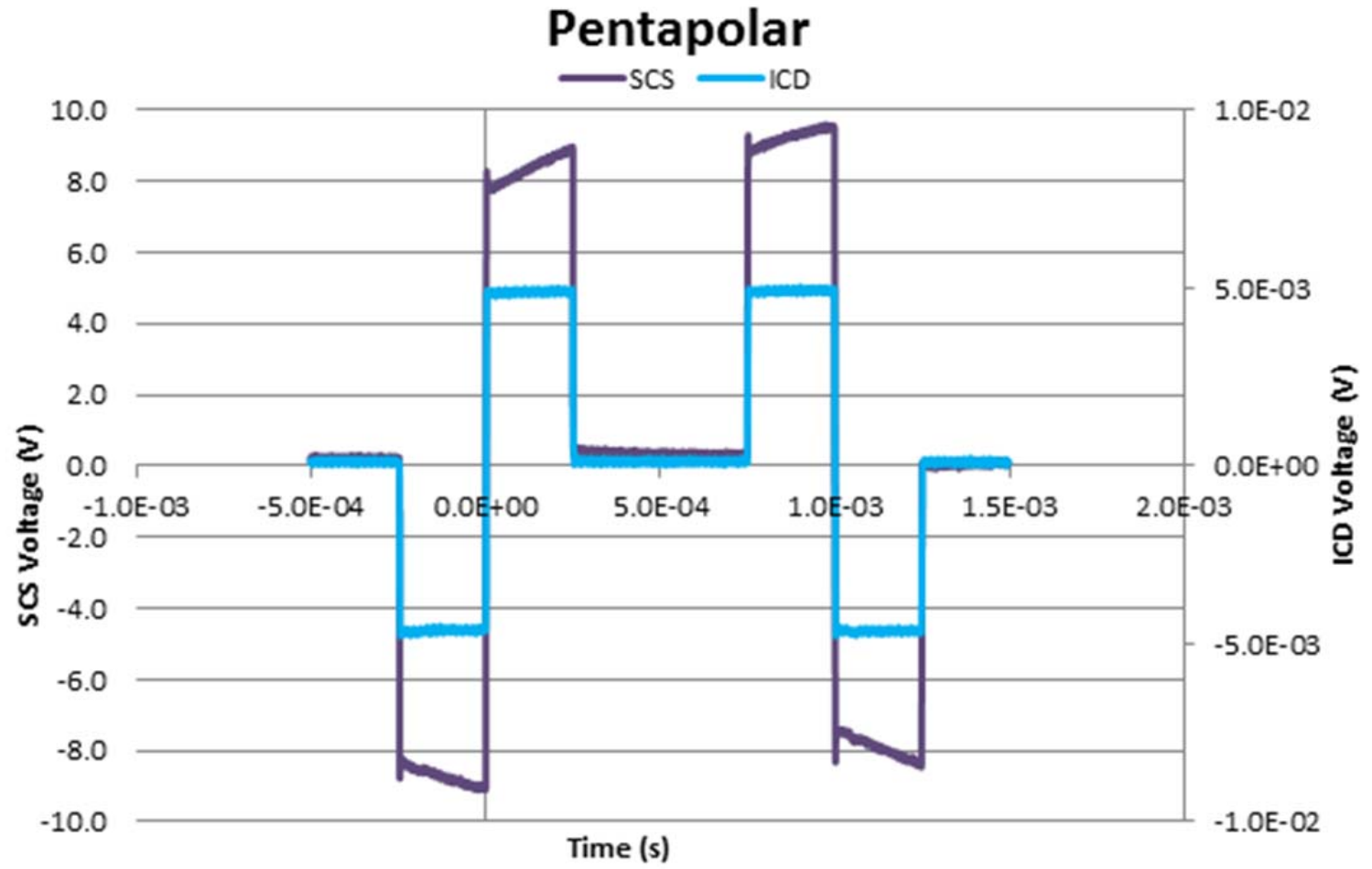

Figure 39: Pentapolar ICD and SCS waveforms before the sense filter is applied (PW $=500 \mu s)$ 


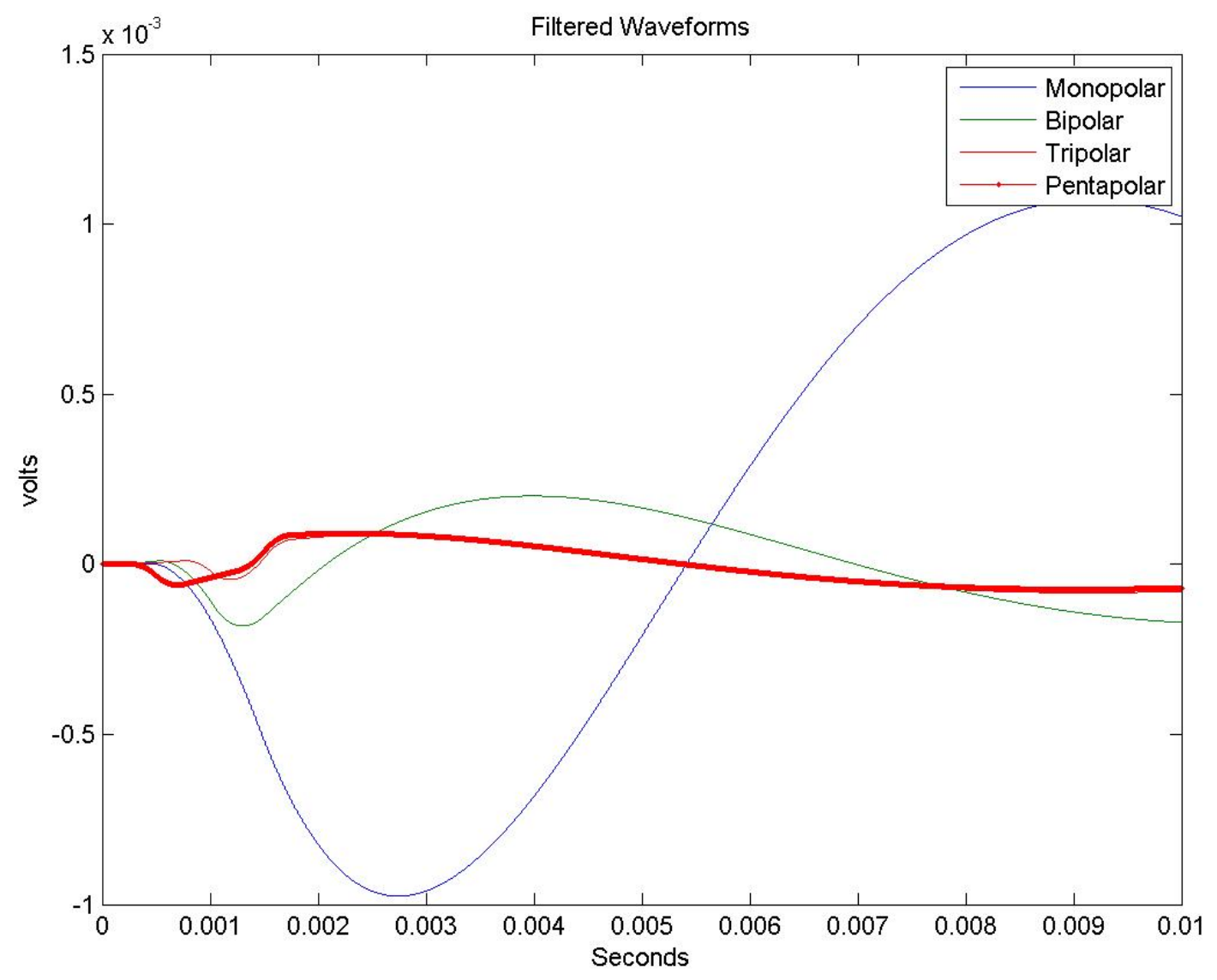

Figure 40: All 4 ICD waveforms post sense filter with a programmed pulse width $=500 \mu \mathrm{s}$ 


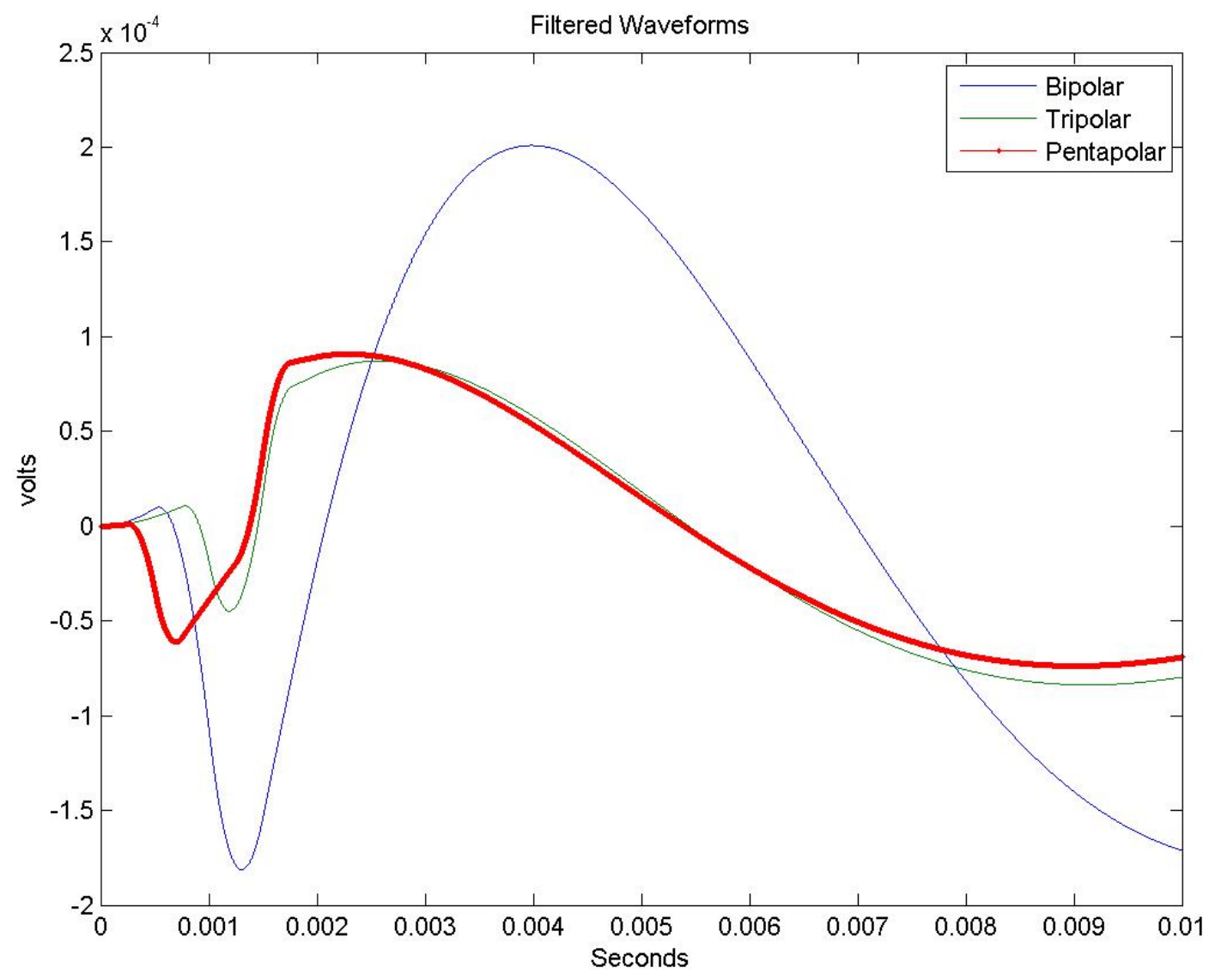

Figure 41: Biphasic, Triphasic, and Pentapolar ICD waveforms post sense filter with a programmed pulse width $=500 \mu \mathrm{s}$ 


\section{Appendix B: Matlab Code}

\section{A. Frequency Domain Functions}

\section{Monophasic.m}

\%\% Monopolar Pulse Calculation (Freq Domain) \%\%

function $[\mathrm{F}, \mathrm{M}, \mathrm{P}]=$ Monopolar(freq, $\mathrm{T})$

$$
\begin{aligned}
& \mathrm{w}=2 * \mathrm{pi} \\
& \mathrm{s}=\mathrm{i} * \mathrm{w} * \text { freq; } \\
& \mathrm{X} 1=1 * \exp \left((\mathrm{T} / 2)^{*} \mathrm{~s}\right) \\
& \mathrm{X} 2=-1 * \exp (-1 *(\mathrm{~T} / 2) * \mathrm{~s}) \\
& \mathrm{F}=(1 / \mathrm{s}) *(\mathrm{X} 1+\mathrm{X} 2) \\
& \mathrm{M}=\operatorname{abs}(\mathrm{F}) ; \\
& \mathrm{P}=\operatorname{angle}(\mathrm{F}) *(180 / \mathrm{pi})
\end{aligned}
$$

end

\section{Biphasic.m}

$\% \%$ Bipolar Pulse Calculation (Freq Domain) \%\%

function $[\mathrm{F}, \mathrm{M}, \mathrm{P}]=$ Bipolar(freq, $\mathrm{T})$

$$
\begin{aligned}
& \mathrm{w}=2 * \mathrm{pi} \\
& \mathrm{s}=\mathrm{i} * \mathrm{w} * \text { freq; } \\
& \mathrm{X} 1=-1 * \exp \left(\mathrm{T}^{*} \mathrm{~s}\right) \\
& \mathrm{X} 2=2 ; \\
& \mathrm{X} 3=-1 * \exp \left(-1 * \mathrm{~T}^{*} \mathrm{~s}\right) \\
& \mathrm{F}=(1 / \mathrm{s}) *(\mathrm{X} 1+\mathrm{X} 2+\mathrm{X} 3) \\
& \mathrm{M}=\operatorname{abs}(\mathrm{F}) ; \\
& \mathrm{P}=\operatorname{angle}(\mathrm{F}) *(180 / \mathrm{pi}) ;
\end{aligned}
$$

end

\section{Triphasic.m}

\%\% Tripolar Pulse Calculation (Freq Domain) \%\%

function $[\mathrm{F}, \mathrm{M}, \mathrm{P}]=$ Tripolar(freq, $\mathrm{T})$ 


$$
\begin{aligned}
& \mathrm{w}=2 * \mathrm{pi} ; \\
& \mathrm{s}=\mathrm{i} * \mathrm{w} * \text { freq; } \\
& \mathrm{X} 1=-1 * \exp \left(2 * \mathrm{~T}^{*} \mathrm{~s}\right) ; \\
& \mathrm{X} 2=2 * \exp \left(\mathrm{T}^{*} \mathrm{~s}\right) ; \\
& \mathrm{X} 3=-2 * \exp \left(-1 * \mathrm{~T}^{*} \mathrm{~s}\right) ; \\
& \mathrm{X} 4=1 * \exp \left(-2 * \mathrm{~T}^{*} \mathrm{~s}\right) ; \\
& \mathrm{F}=(1 / \mathrm{s}) *(\mathrm{X} 1+\mathrm{X} 2+\mathrm{X} 3+\mathrm{X} 4) ; \\
& \mathrm{M}=\operatorname{abs}(\mathrm{F}) ; \\
& \mathrm{P}=\operatorname{angle}(\mathrm{F}) *(180 / \mathrm{pi}) ;
\end{aligned}
$$

end

\section{Triphasic_Delay.m}

$\% \%$ Bipolar Pulse Calculation (Freq Domain) \%\%

function $[\mathrm{F}, \mathrm{M}, \mathrm{P}]=$ Tripolar_Delay $($ freq, $\mathrm{T}, \mathrm{D})$

$$
\begin{aligned}
& \mathrm{w}=2 * \mathrm{pi} \\
& \mathrm{s}=\mathrm{i} * \mathrm{w} * \text { freq; } \\
& \mathrm{X} 1=-1 * \exp (((2 * \mathrm{~T})+\mathrm{D}) * \mathrm{~s}) ; \\
& \mathrm{X} 2=1 * \exp ((\mathrm{T}+\mathrm{D}) * \mathrm{~s}) ; \\
& \mathrm{X} 3=1 * \exp (\mathrm{T} * \mathrm{~s}) ; \\
& \mathrm{X} 4=-1 * \exp (-1 * \mathrm{~T} * \mathrm{~s}) ; \\
& \mathrm{X} 5=-1 * \exp (-1 *(\mathrm{~T}+\mathrm{D}) * \mathrm{~s}) ; \\
& \mathrm{X} 6=1 * \exp (-1 *((2 * \mathrm{~T})+\mathrm{D}) * \mathrm{~s}) ; \\
& \mathrm{F}=(1 / \mathrm{s}) *(\mathrm{X} 1+\mathrm{X} 2+\mathrm{X} 3+\mathrm{X} 4+\mathrm{X} 5+\mathrm{X} 6) ; \\
& \mathrm{M}=\operatorname{abs}(\mathrm{F}) ; \\
& \mathrm{P}=\operatorname{angle}(\mathrm{F}) *(180 / \mathrm{pi}) ;
\end{aligned}
$$

end

\section{Pentapolar.m}

$\% \%$ Bipolar Pulse Calculation (Freq Domain) \%\%

function $[\mathrm{F}, \mathrm{M}, \mathrm{P}]=$ Pentapolar(freq, $\mathrm{T})$

$$
\begin{aligned}
& \mathrm{w}=2 * \mathrm{pi} \\
& \mathrm{s}=\mathrm{i} * \mathrm{w} * \text { freq; } \\
& \mathrm{X} 1=-1 * \exp (3 * \mathrm{~T} * \mathrm{~s}) \\
& \mathrm{X} 2=2 * \exp \left(2 * \mathrm{~T}^{*} \mathrm{~s}\right) \\
& \mathrm{X} 3=-1 * \exp \left(\mathrm{T}^{*} \mathrm{~s}\right)
\end{aligned}
$$




$$
\begin{aligned}
& \mathrm{X} 4=1 * \exp (-1 * \mathrm{~T} * \mathrm{~s}) ; \\
& \mathrm{X} 5=-2 * \exp (-2 * \mathrm{~T} * \mathrm{~s}) \\
& \mathrm{X} 6=1 * \exp (-3 * \mathrm{~T} * \mathrm{~s}) ; \\
& \mathrm{F}=(1 / \mathrm{s}) *(\mathrm{X} 1+\mathrm{X} 2+\mathrm{X} 3+\mathrm{X} 4+\mathrm{X} 5+\mathrm{X} 6) \\
& \mathrm{M}=\operatorname{abs}(\mathrm{F}) \\
& \mathrm{P}=\operatorname{angle}(\mathrm{F}) *(180 / \mathrm{pi})
\end{aligned}
$$

end

\section{B. Matlab Test Code}

\section{No Delay Waveform Comparison}

clf; \% clear the plot

plot $={ }^{~ ' T}=500$ us $^{\prime} ; \%$ Used in plot Title

$\%$ Variables

$\mathrm{T}=500 ; \quad \%$ in us

$\operatorname{Min}=1 ; \quad \%$ in $\mathrm{Hz}$

$\operatorname{Max}=250 ; \quad \%$ in $\mathrm{Hz}$

Mag_min $=10 \mathrm{E}-13 ; \%$ in $\mathrm{dB}$

Mag_max $=10 \mathrm{E}-4 ; \%$ in $\mathrm{dB}$

Res $=1 ; \quad \%$ in $\mathrm{Hz}$

Delay $=0 ; \quad \%$ in us

$\%$ Variable Conversions

$\mathrm{T}=\mathrm{T} * 1 \mathrm{E}-6 ; \quad \%$ Convert to us

Delay $=$ Delay $* 1 \mathrm{E}-6 ; \quad \%$ Convert to us

ArraySize $=\operatorname{round}((\operatorname{Max}-$ Min $) /$ Res $)+1$;

$\%$ Array Allocation

$\%$ Vector

$\mathrm{Fm}=\operatorname{zeros}(1$, ArraySize $)$;

$\mathrm{Fb}=\operatorname{zeros}(1$, ArraySize);

$\mathrm{Ft}=$ zeros(1, ArraySize);

$\mathrm{Fp}=\operatorname{zeros}(1$, ArraySize $)$;

$\%$ Magnitude

$\mathrm{Mm}=\operatorname{zeros}(1$, ArraySize $)$

$\mathrm{Mb}=\operatorname{zeros}(1$, ArraySize $)$;

$\mathrm{Mt}=\operatorname{zeros}(1$, ArraySize $)$;

$\mathrm{Mp}=\operatorname{zeros}(1$, ArraySize $)$;

$\%$ Phase

Pm $=$ zeros(1, ArraySize);

$\mathrm{Pb}=\operatorname{zeros}(1$, ArraySize $)$; 


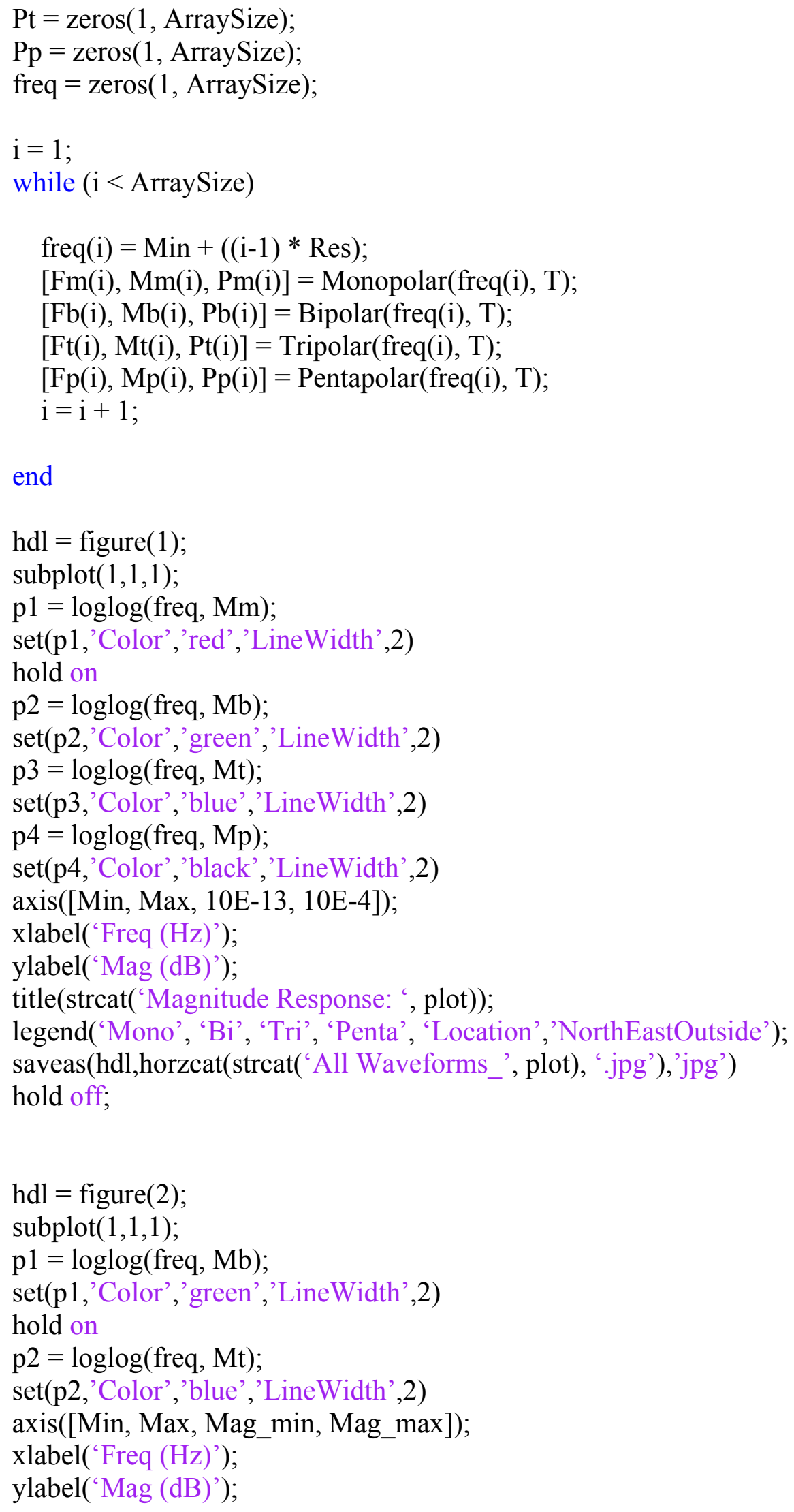


title(strcat('Magnitude Response: ', plot)); legend('Bi', 'Tri', 'Location', 'NorthEastOutside');

saveas(hdl,horzcat(strcat('Tri_vs_Bi_',plot), '.jpg'),'jpg')

\section{Triphasic Delay Waveform Comparison}

clf; $\%$ clear the plot

plot $={ }^{`} \mathrm{~T}=50$ us'; \% Used in plot Title

$\%$ Variables

$\mathrm{T}=50 ; \quad \%$ in us

$\operatorname{Min}=1 ; \quad \%$ in $\mathrm{Hz}$

$\operatorname{Max}=250 ; \quad \%$ in $\mathrm{Hz}$

Mag_min $=10 \mathrm{E}-14 ; \%$ in $\mathrm{dB}$

Mag_max $=10 \mathrm{E}-6 ; \quad \%$ in $\mathrm{dB}$

Res $=1 ; \quad \%$ in $\mathrm{Hz}$

Delay $=1 ; \quad \%$ in us

$\%$ Variable Conversions

$\mathrm{T}=\mathrm{T} * 1 \mathrm{E}-6 ; \quad \%$ Convert to us

Delay $=$ Delay $* 1 \mathrm{E}-6 ; \quad \%$ Convert to us

ArraySize $=\operatorname{round}((\operatorname{Max}-\operatorname{Min}) / \operatorname{Res})+1$;

$\%$ Array Allocation

$\%$ Vector

$\mathrm{Ft} 1=\operatorname{zeros}(1$, ArraySize $)$;

Ft2 $=$ zeros(1, ArraySize);

Ft3 = zeros(1, ArraySize);

$\%$ Magnitude

Mt1 = zeros $(1$, ArraySize $) ;$

$\mathrm{Mt} 2=\operatorname{zeros}(1$, ArraySize $) ;$

$\mathrm{Mt} 3=\operatorname{zeros}(1$, ArraySize $)$;

$\%$ Phase

Pt1 = zeros(1, ArraySize);

$\mathrm{Pt} 2=\operatorname{zeros}(1$, ArraySize $)$;

Pt3 = zeros (1, ArraySize $)$;

freq $=$ zeros $(1$, ArraySize $)$;

$\mathrm{i}=1$;

while $(\mathrm{i}<$ ArraySize)

freq(i) $=\operatorname{Min}+((\mathrm{i}-1) *$ Res $)$;

[Ft1(i), Mt1(i), Pt1(i)] = Tripolar(freq(i), T);

[Ft2(i), Mt2(i), Pt2(i)] = Tripolar_Delay(freq(i), T, Delay);

[Ft3(i), Mt3(i), Pt3(i)] = Tripolar_Delay(freq(i), T, Delay*10); 
$\mathrm{i}=\mathrm{i}+1$;

end

hdl = figure $(1)$;

subplot $(1,1,1)$;

p1 = $\log \log ($ freq, Mt1);

set(p1,'Color','red','LineWidth',2)

hold all

p2 $=\log \log ($ freq, Mt2);

set(p2,'Color','green', 'LineWidth',2)

p3 = $\log \log ($ freq, Mt3);

set(p3,'Color', 'blue','LineWidth',2)

axis([Min, Max, Mag_min, Mag_max]);

xlabel('Hz');

ylabel('Mag');

title(strcat('Magnitude Response: ', plot));

legend('No Delay', '1us Delay', '10us Delay', 'Location', 'NorthEastOutside');

saveas(hdl,horzcat(strcat('Tri_Delay_, plot), '.jpg'),'jpg')

\section{ICD Sense Filter}

clear all; close all;

$\% \%$ Import Data

monopolar $=$ xlsread $\left({ }^{\circ}\right.$ Monopolar.csv', 'C:C');

bipolar $=$ xlsread('Bipolar.csv', 'C:C');

tripolar $=$ xlsread ('Tripolar.csv', 'C:C');

pentapolar $=\operatorname{xlsread}\left({ }^{' P e n t a p o l a r}\right.$. csv', $\left.^{\prime} \mathrm{C}: \mathrm{C}^{\prime}\right)$;

$\% \%$ User Input

Experiment $={ }^{' S a l i n e ~ T a n k ~}(1.90 \mathrm{mS}$ per $\mathrm{cm}) '$;

duration $=50000 ; \%$ duration in microseconds

$\mathrm{fs}=5 \mathrm{E} 6 ; \quad \%$ Sampling Frequency (in $\mathrm{Hz}$ )

$\mathrm{f0}=50 ; \quad \%$ Center Frequency (BPF)

$\mathrm{Q}=0.9 ; \% 0.365 ; \quad \%$ Q-value $(\mathrm{BPF})$

$\mathrm{fc}=10 ; \quad \%$ Cutoff Frequency (HPF)

$\%$ Start $=0 ; \quad \%$ start is in microseconds

$\mathrm{PW}=500 ; \quad \%$ pulse width (for chart labels)

$Y \max =0.0007$

$Y \min =-0.0007$

$\% \%$ Main

egm $=$ zeros(duration, 1$)$;

storedWaveforms $=$ zeros(duration, 4$)$; 
waveform $\{1,1\}=$ 'mono'; waveform $\{2,1\}=$ 'bi'; waveform $\{3,1\}=$ 'tri'; waveform $\{4,1\}=$ 'penta';

for inx $=1: 4$

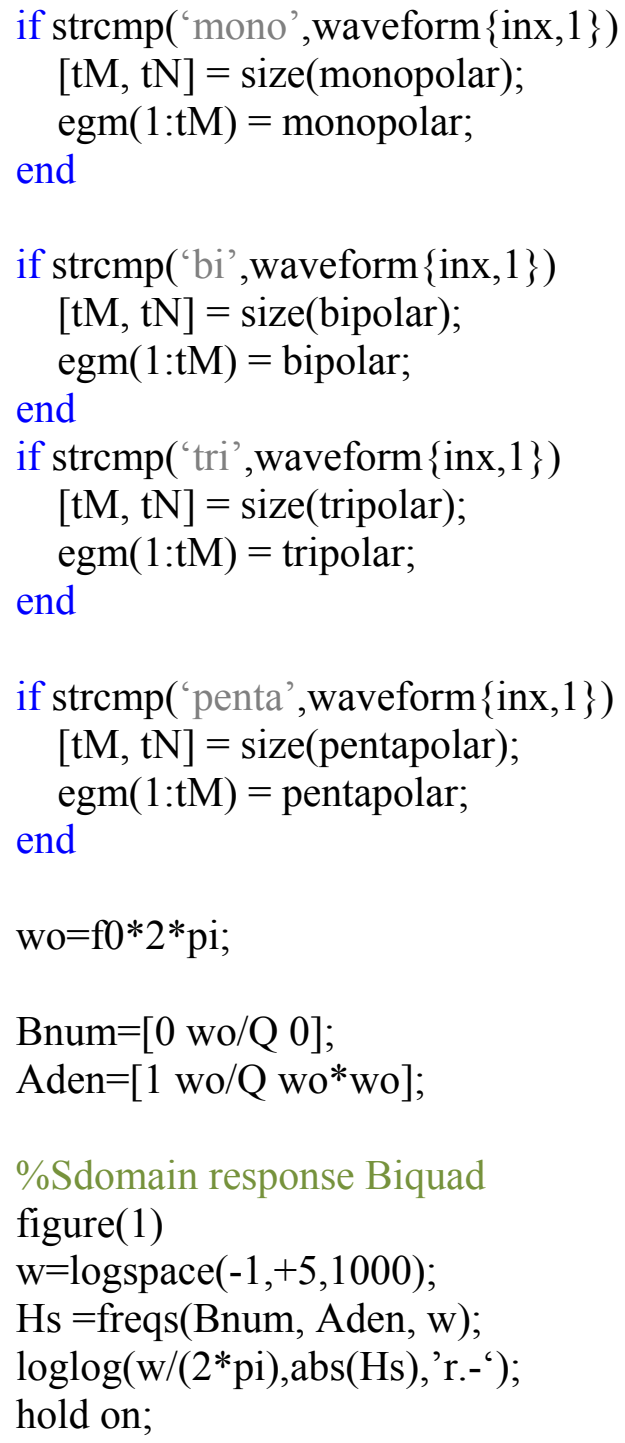


$\mathrm{B}=\left[\begin{array}{ll}1 & 0\end{array}\right]$;

$\mathrm{A}=[1 \mathrm{wh}]$;

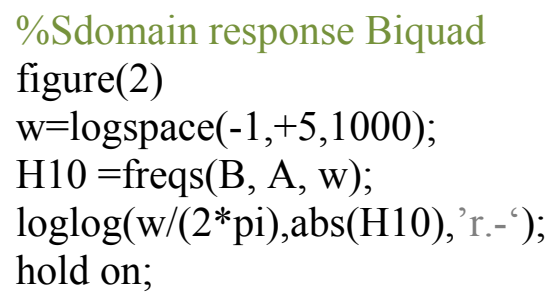

storedWaveforms $(:$, inx $)=$ gain*y 00

plot(t,storedWaveforms(:,inx $\left.),{ }^{\prime} r .-^{-}\right)$;

if $\operatorname{strcmp}$ ('mono', waveform $\{$ inx, 1$\}$ )

Yminmax $=$ [Ymin ,Ymax $]$;

titl $=$ horzcat(waveform $\{$ inx, 1$\}$,'phasic $\mathrm{PW}={ }^{\prime}$, num2str(PW)); 


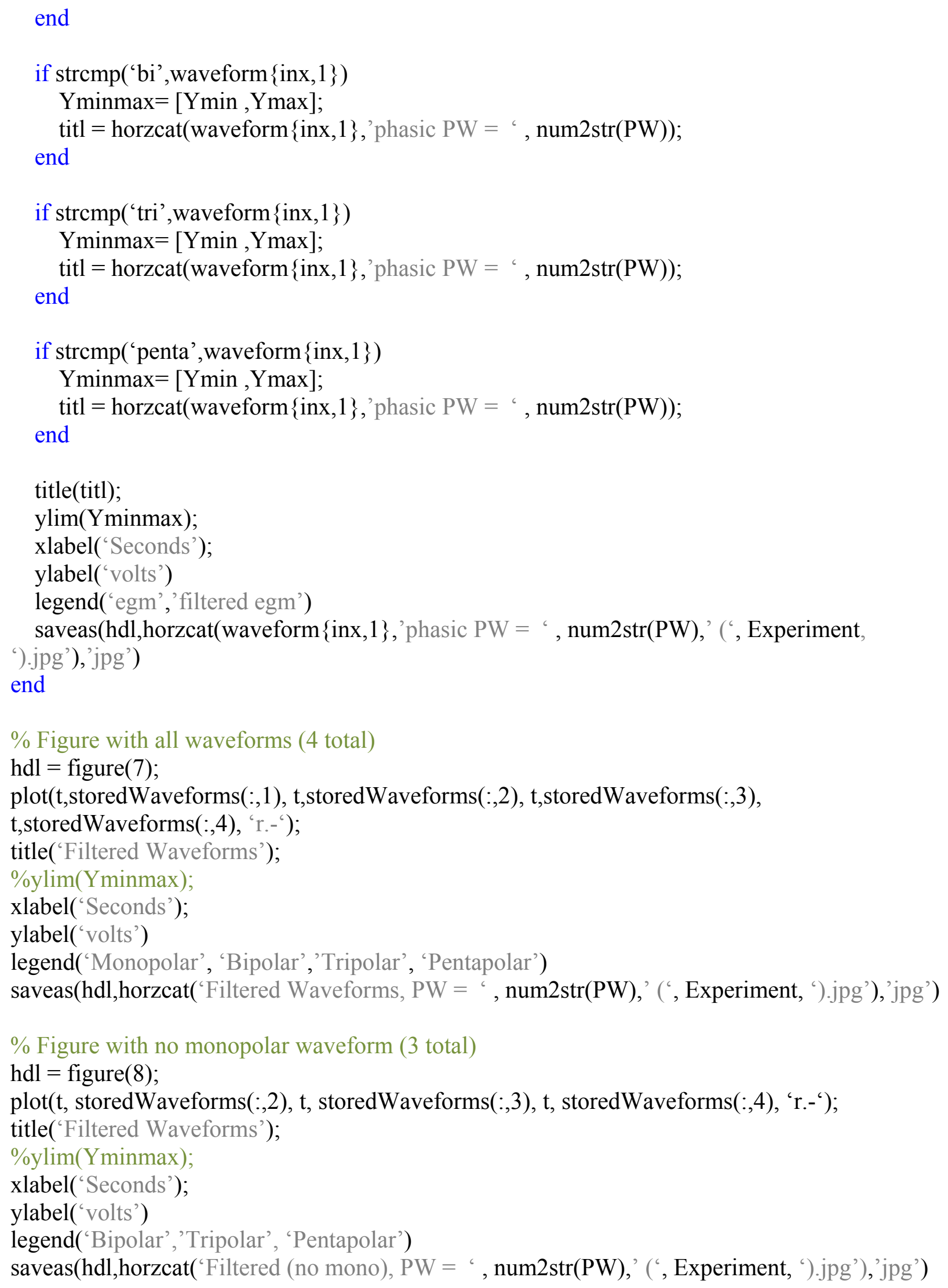




\section{Appendix C: Pulse Generator Firmware Code}

All code was written in AtmelStudio 6.0 I. The firmware was downloaded and run on the microcontroller by using the AVRISP mkII compatible PDI programmer (MattairTech, 2012). Files in the project:

- "main.c"

- "GeneralFunctions.c"

- "Communication.c"

- "Timers.c"

- "Definitions.h"

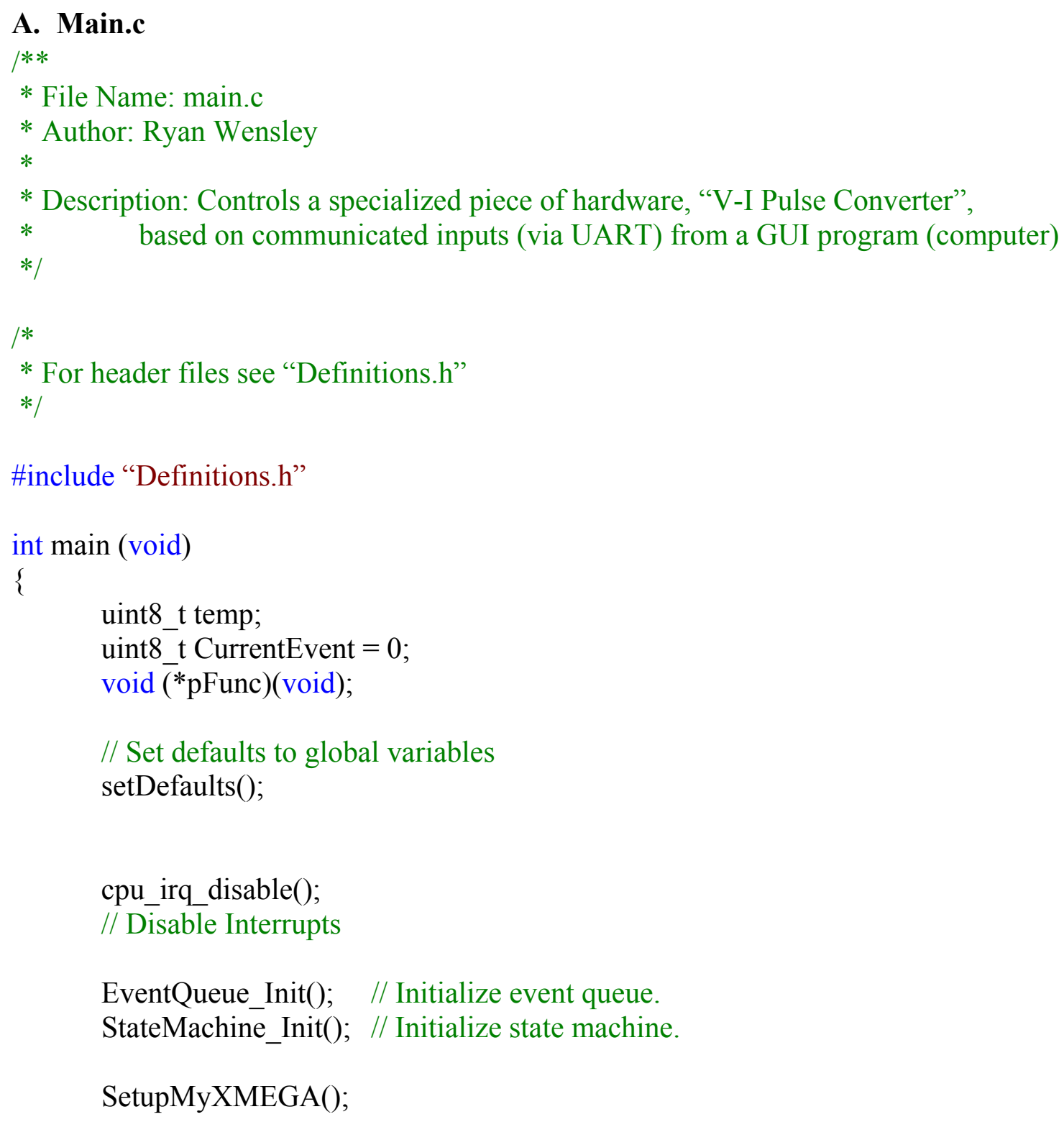




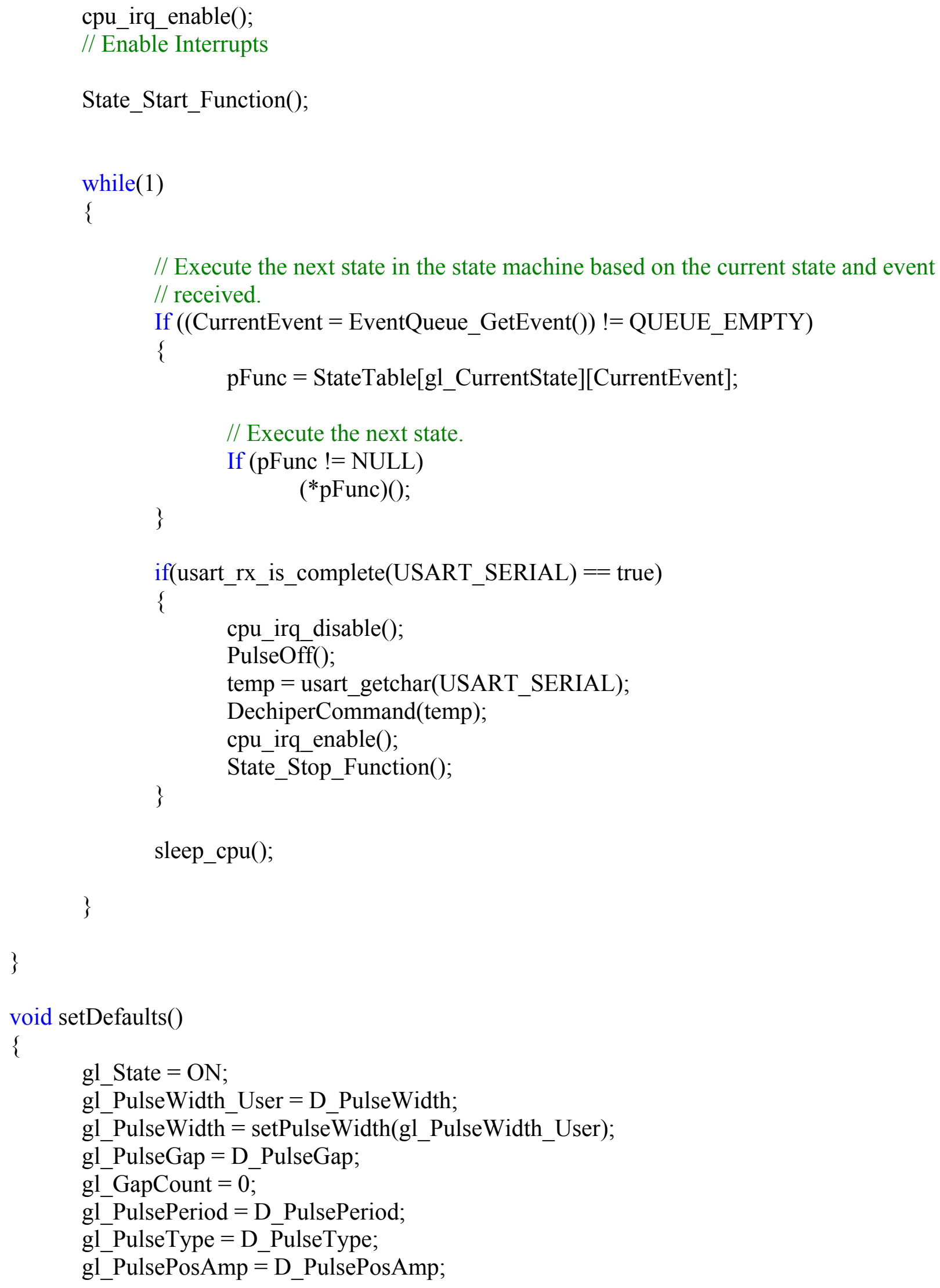




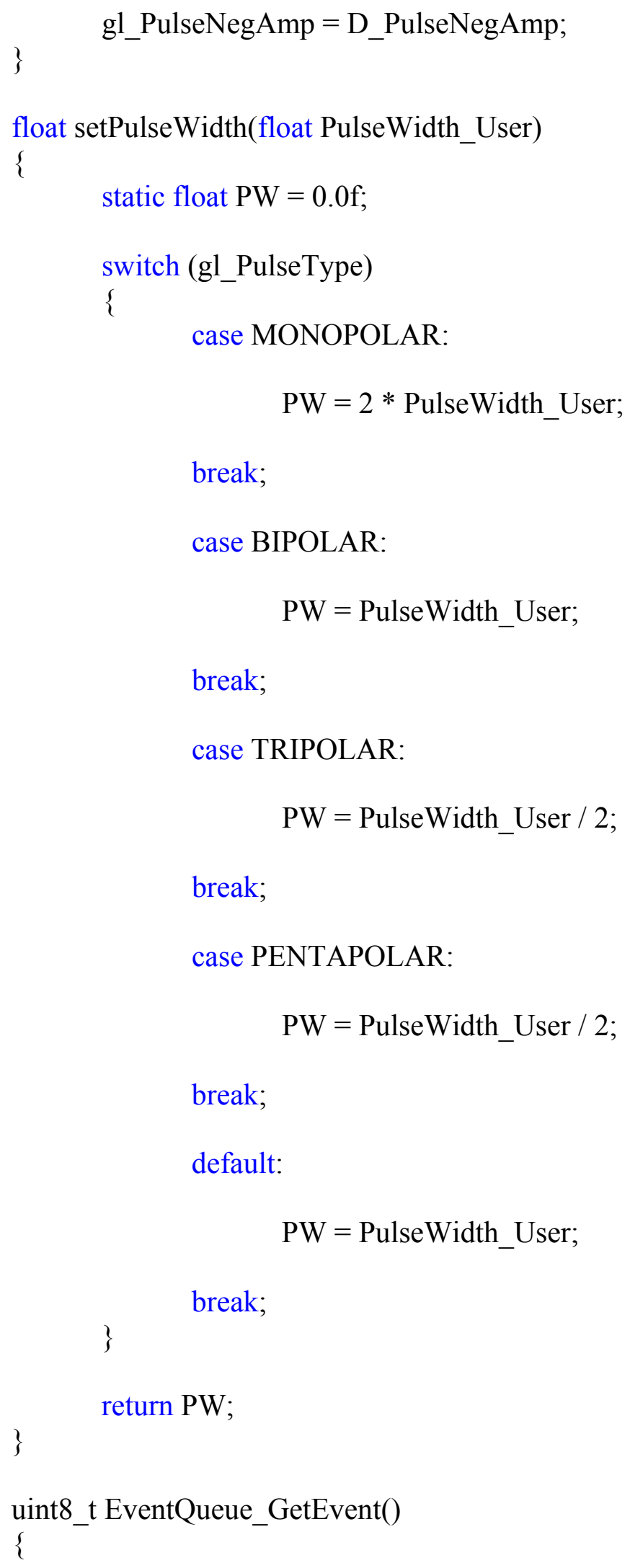




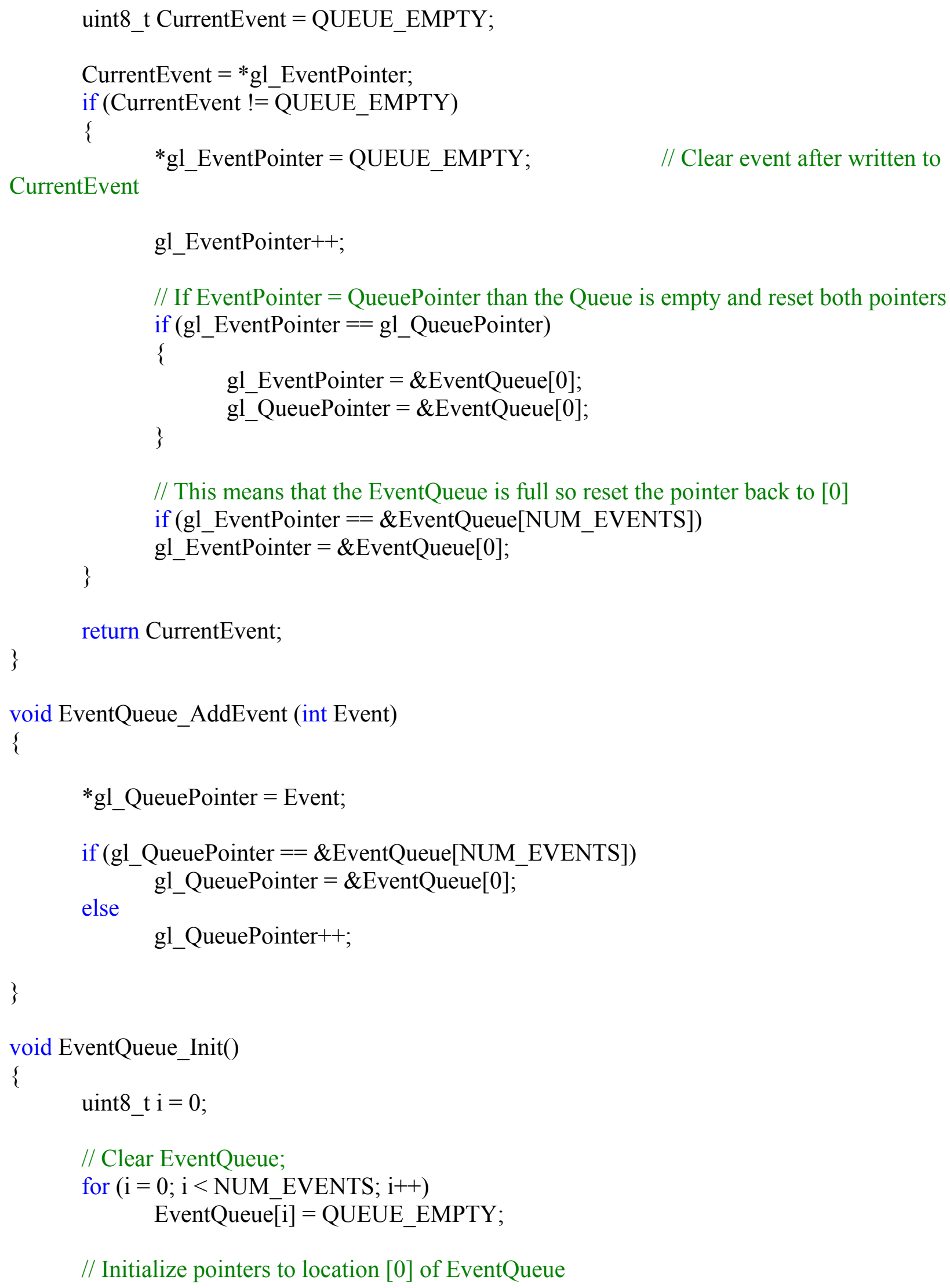




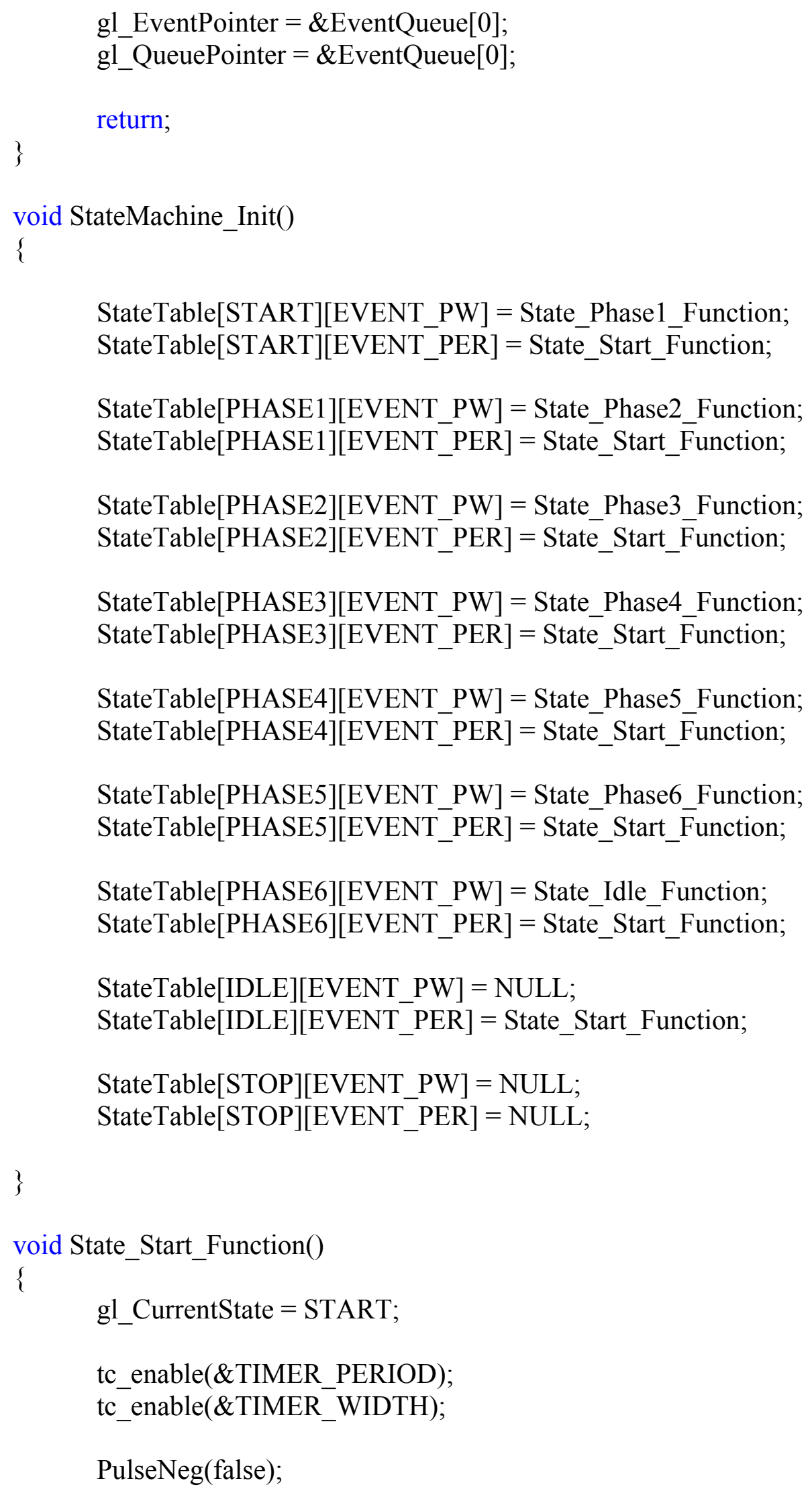




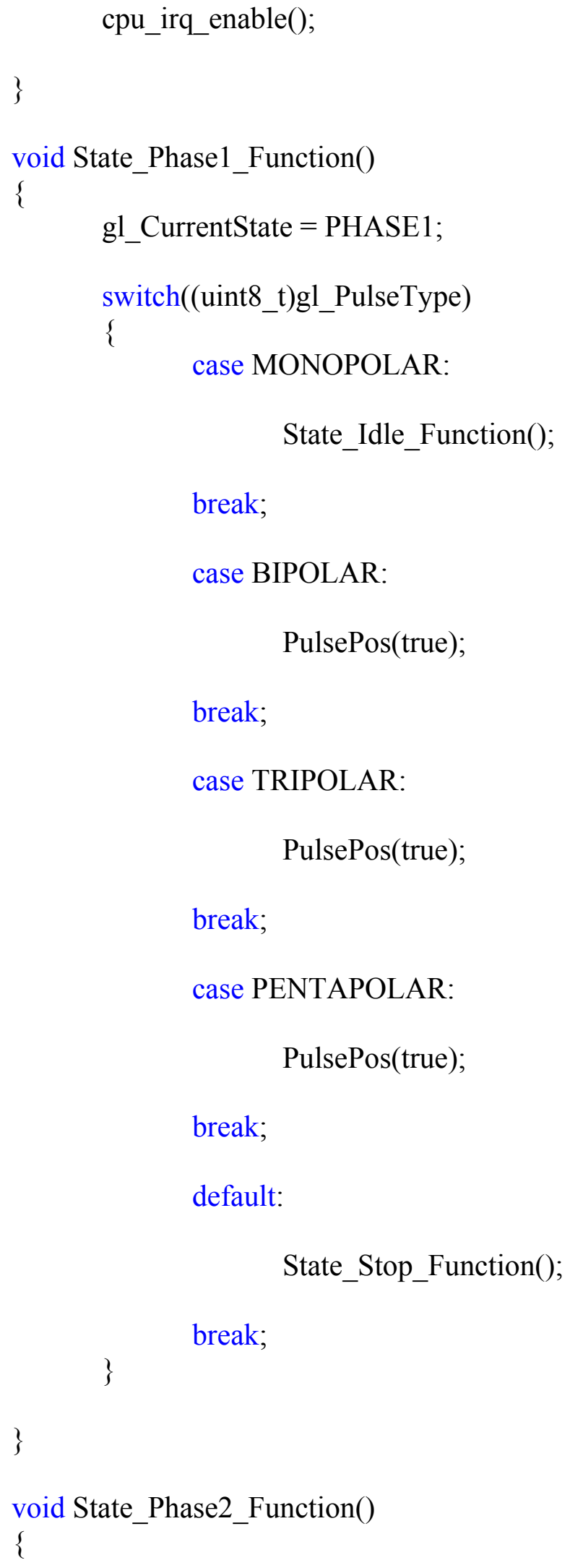




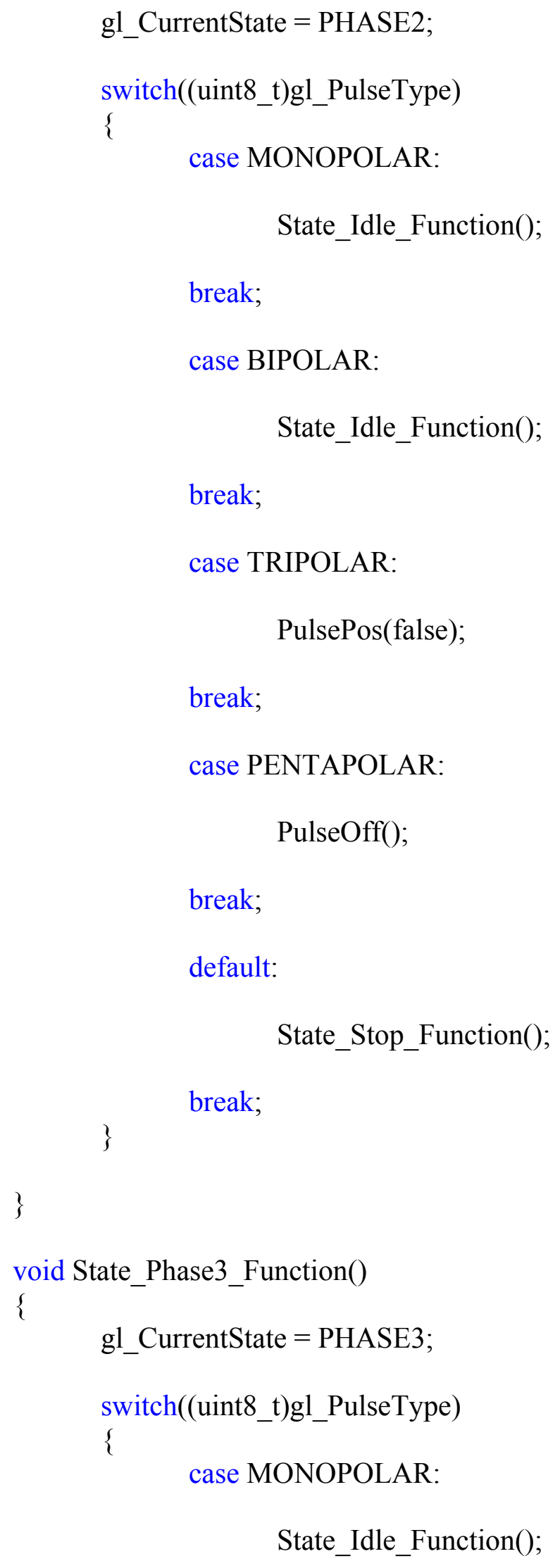

State_Idle_Function(); 


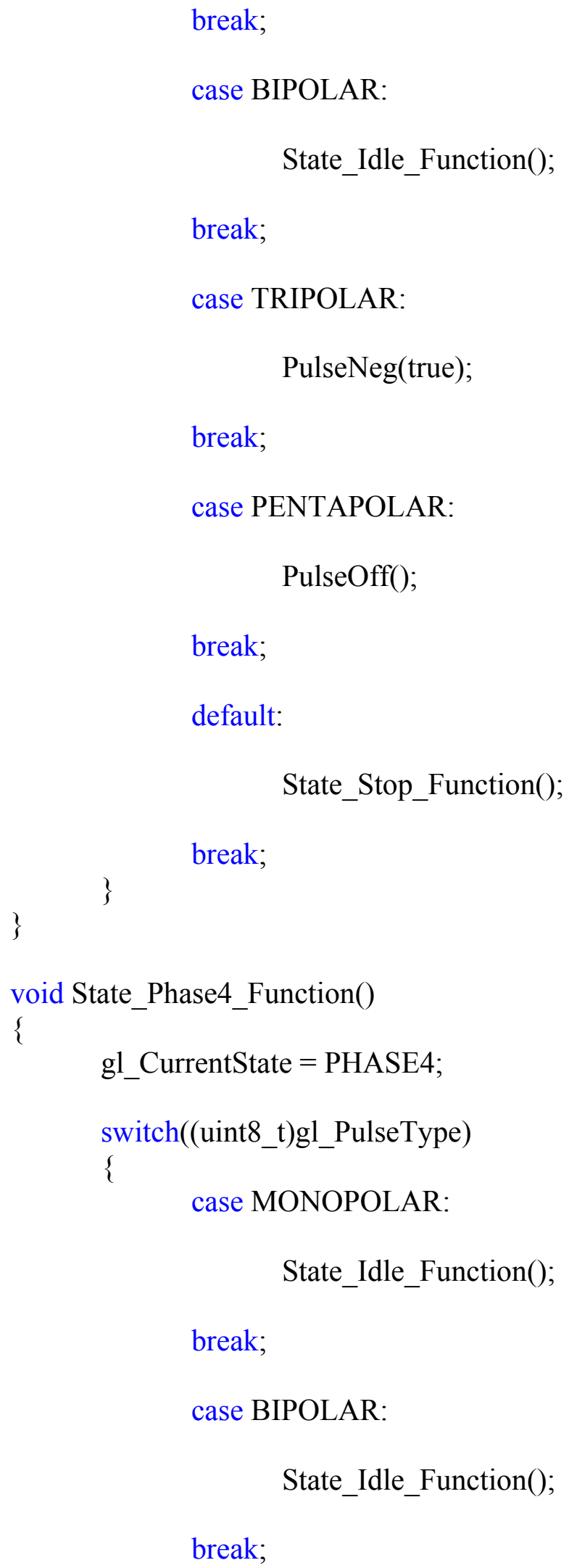




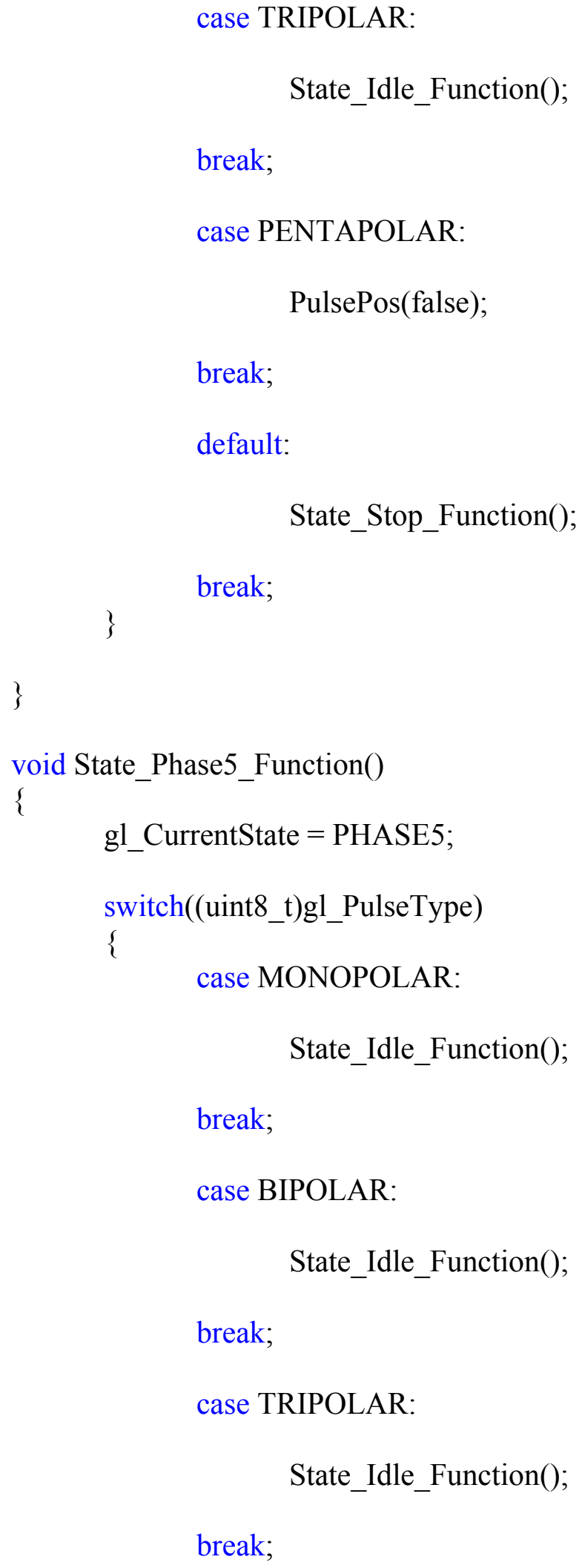


case PENTAPOLAR:

PulseNeg(true);

break;

default:

State_Stop_Function();

\} break;

void State_Phase6_Function()

\{

gl_CurrentState = PHASE6;

switch((uint8_t)gl_PulseType)

\{

case MONOPOLAR:

State_Idle_Function();

break;

case BIPOLAR:

State_Idle_Function();

break;

case TRIPOLAR:

State_Idle_Function();

break;

case PENTAPOLAR:

PulseOff();

break;

default: 


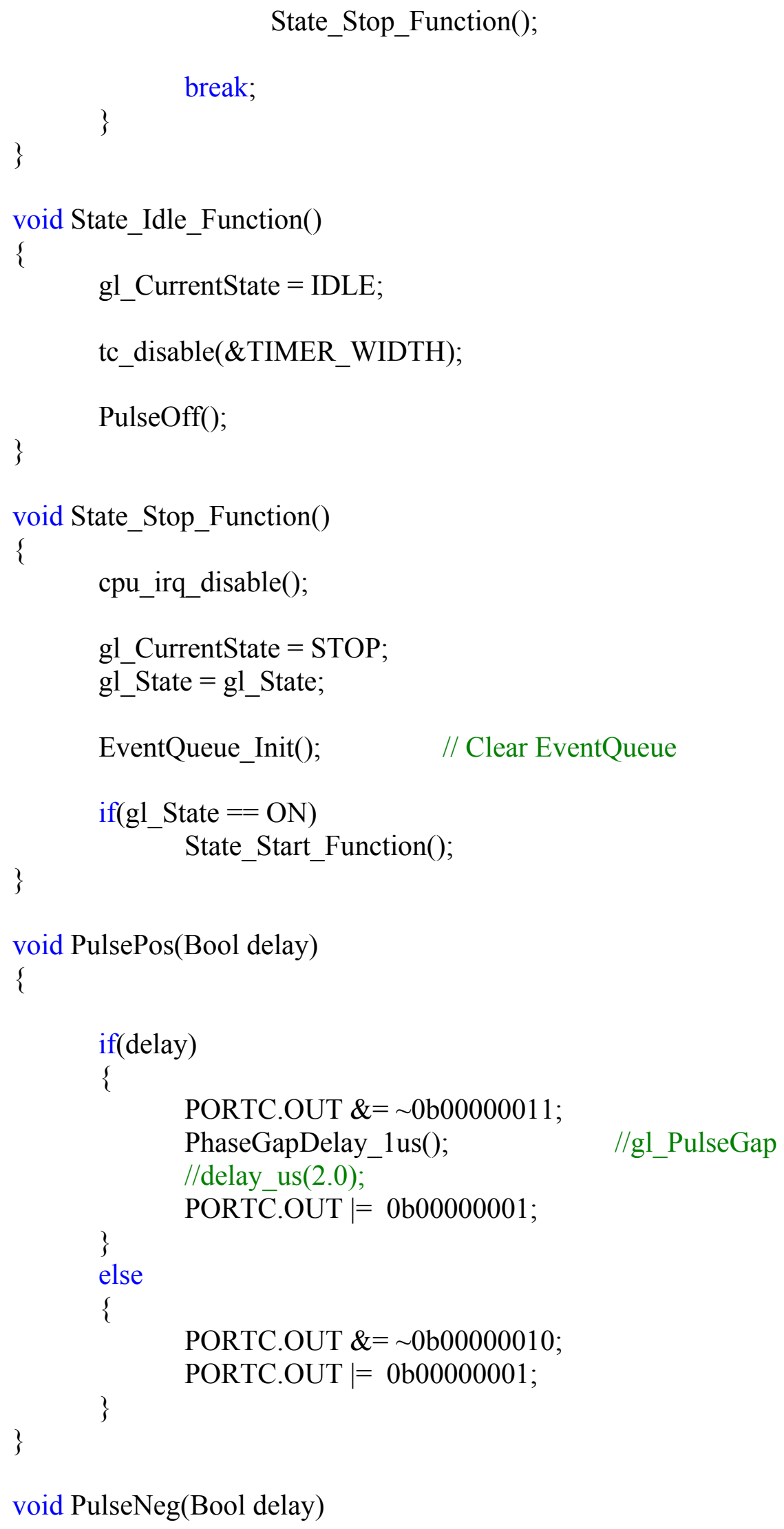




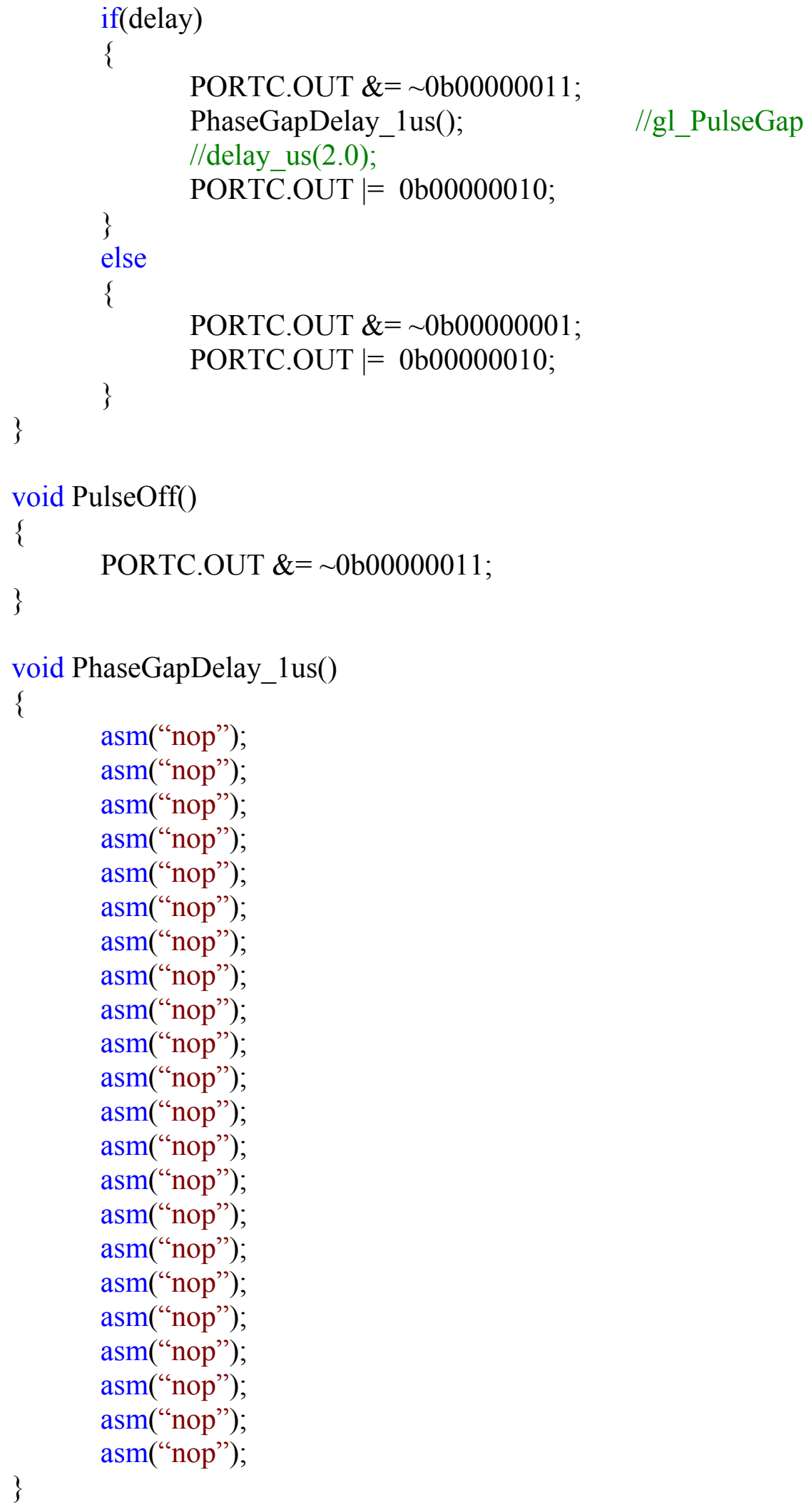




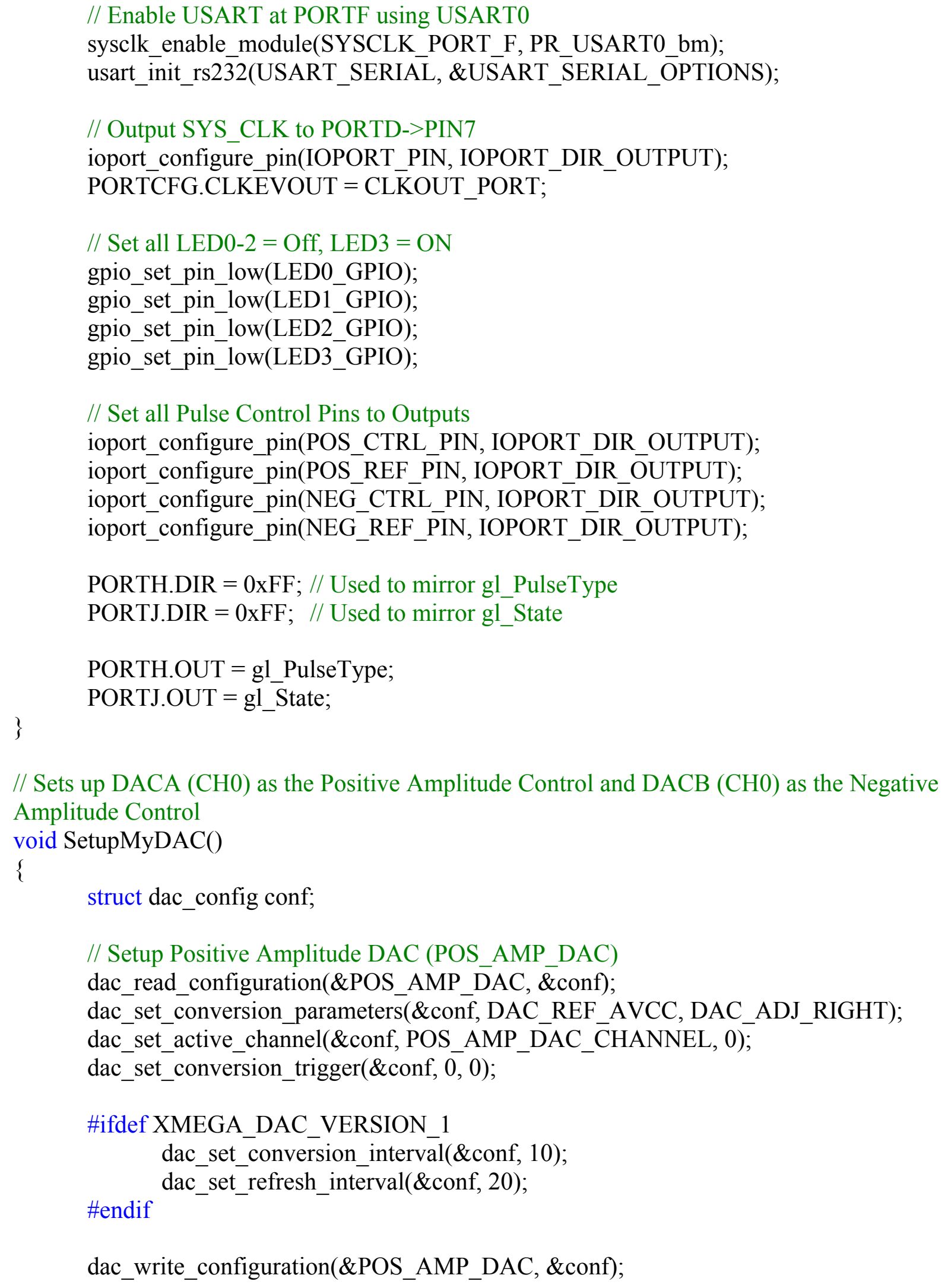




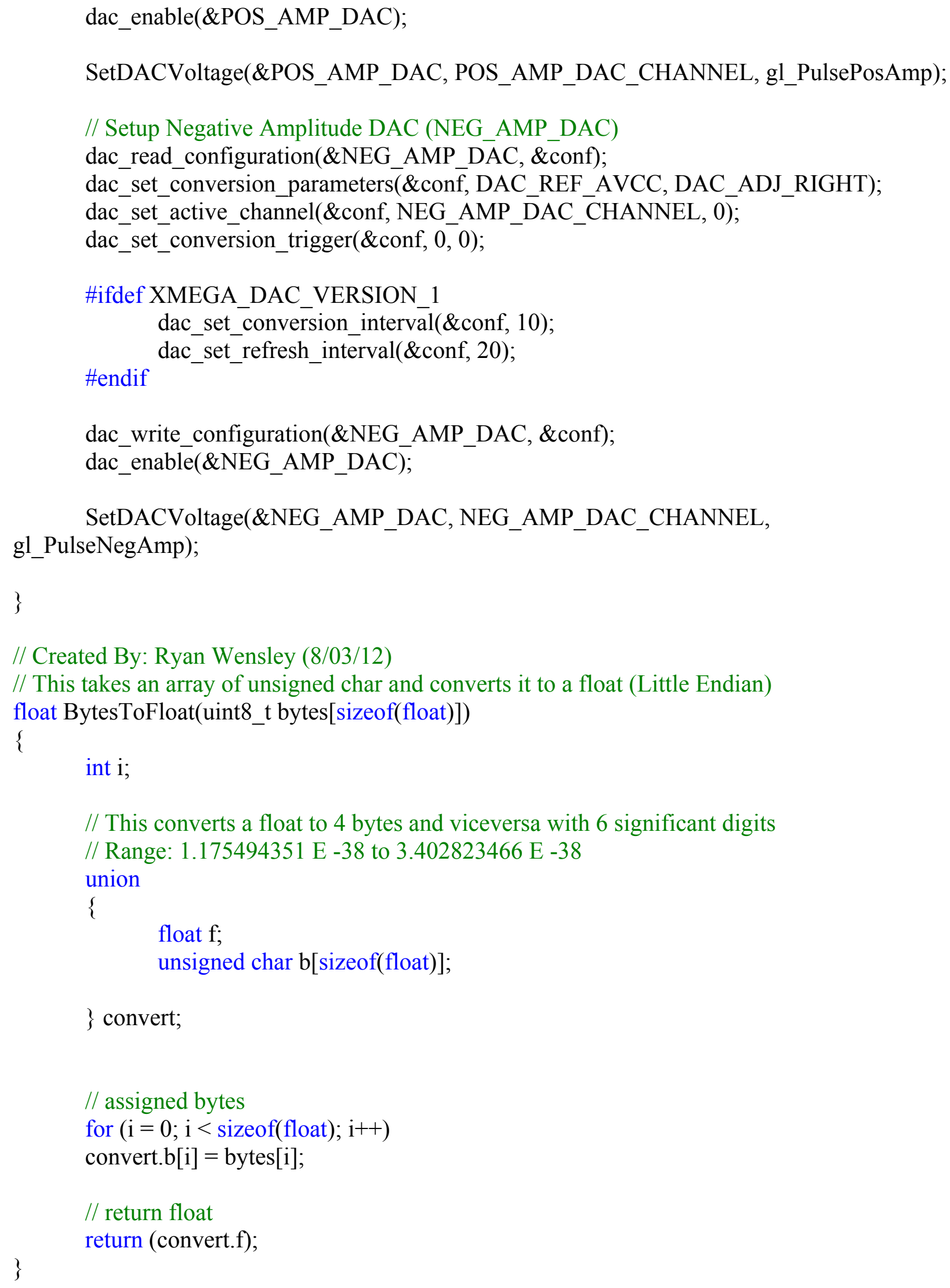


// Created By: Ryan Wensley (8/03/12)

// This takes a float decimal number and converts it to an array of unsigned char (Little Endian)

// It returns a float to compare your input to your output to make sure there is no conversion error float FloatToBytes(float var, uint8_t bytes[sizeof(float)])

\{

$$
\text { int } \mathrm{i}=0 \text {; }
$$

// This converts a float to 4 bytes and viceversa with 6 significant digits

// Range: $1.175494351 \mathrm{E}-38$ to $3.402823466 \mathrm{E}-38$

union

\{

float f;

unsigned char b[sizeof(float)];

\} convert;

// assigned float

convert. $\mathrm{f}=$ var;

// Record bytes

for $(\mathrm{i}=0 ; \mathrm{i}<\operatorname{sizeof(float)} ; \mathrm{i}++$ )

bytes $[\mathrm{i}]=$ convert.b[i];

// return array of bytes

return (convert.f);

\}

float SetDACVoltage(DAC_t* dac, uint8_t ch_mask, float desiredCurrent)

\{

static float slope $=1.0$;

static float offset $=0.0$;

static float resistor $=120$;

if $(\mathrm{dac}==\&$ POS AMP DAC $)$

\{

slope $=$ POS_DAC_SLOPE;

offset $=$ POS_DAC_OFFSET;

resistor $=$ POS_DAC $\bar{C} \_$RESISTOR;

\}

else if $\left(\mathrm{dac}==\& N E G \_A M P \_D A C\right)$

\{

slope $=$ NEG_DAC_SLOPE;

offset $=$ NEG DAC OFFSET;

resistor $=$ NEG_DAC $\bar{C} \_$RESISTOR; 


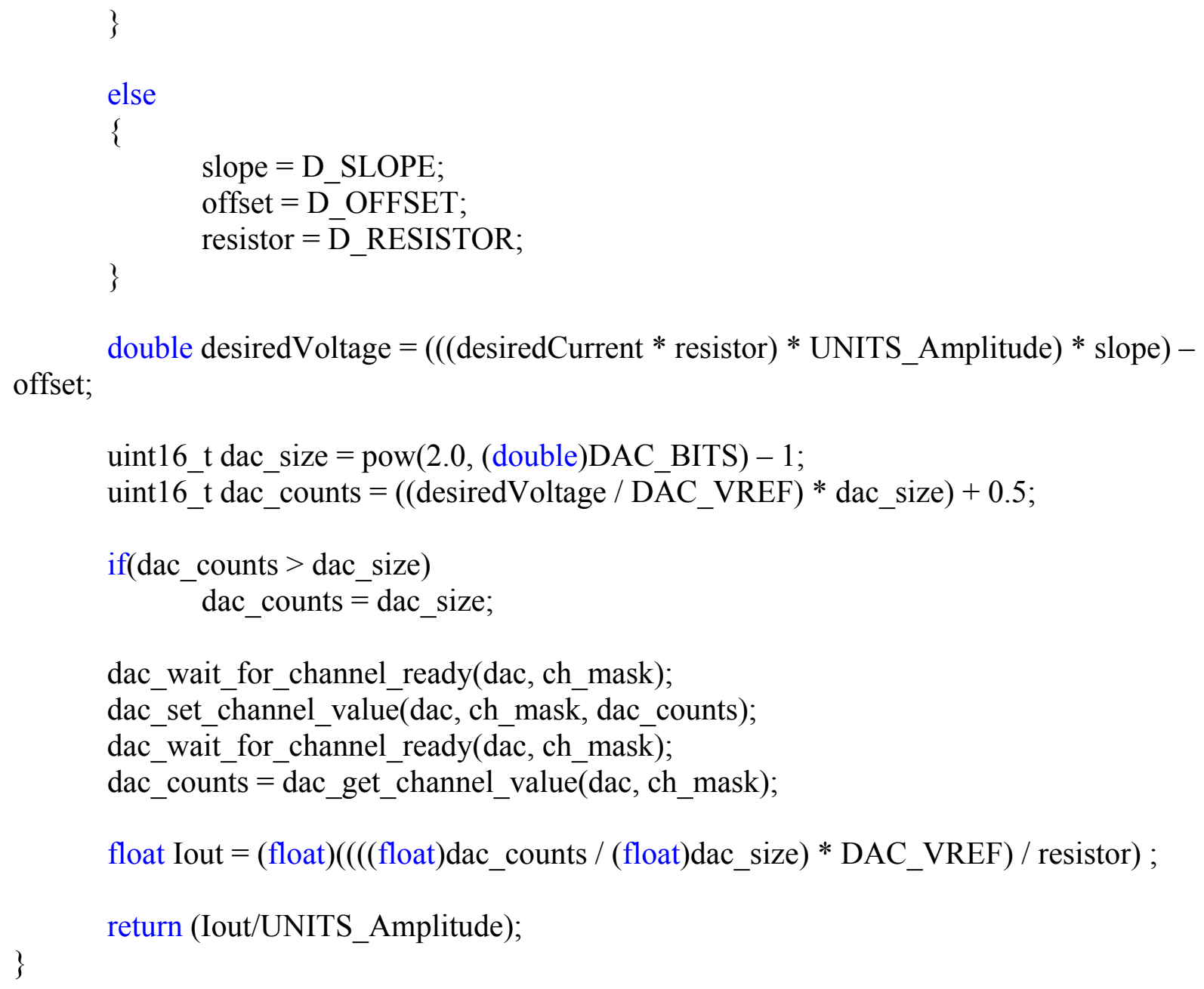




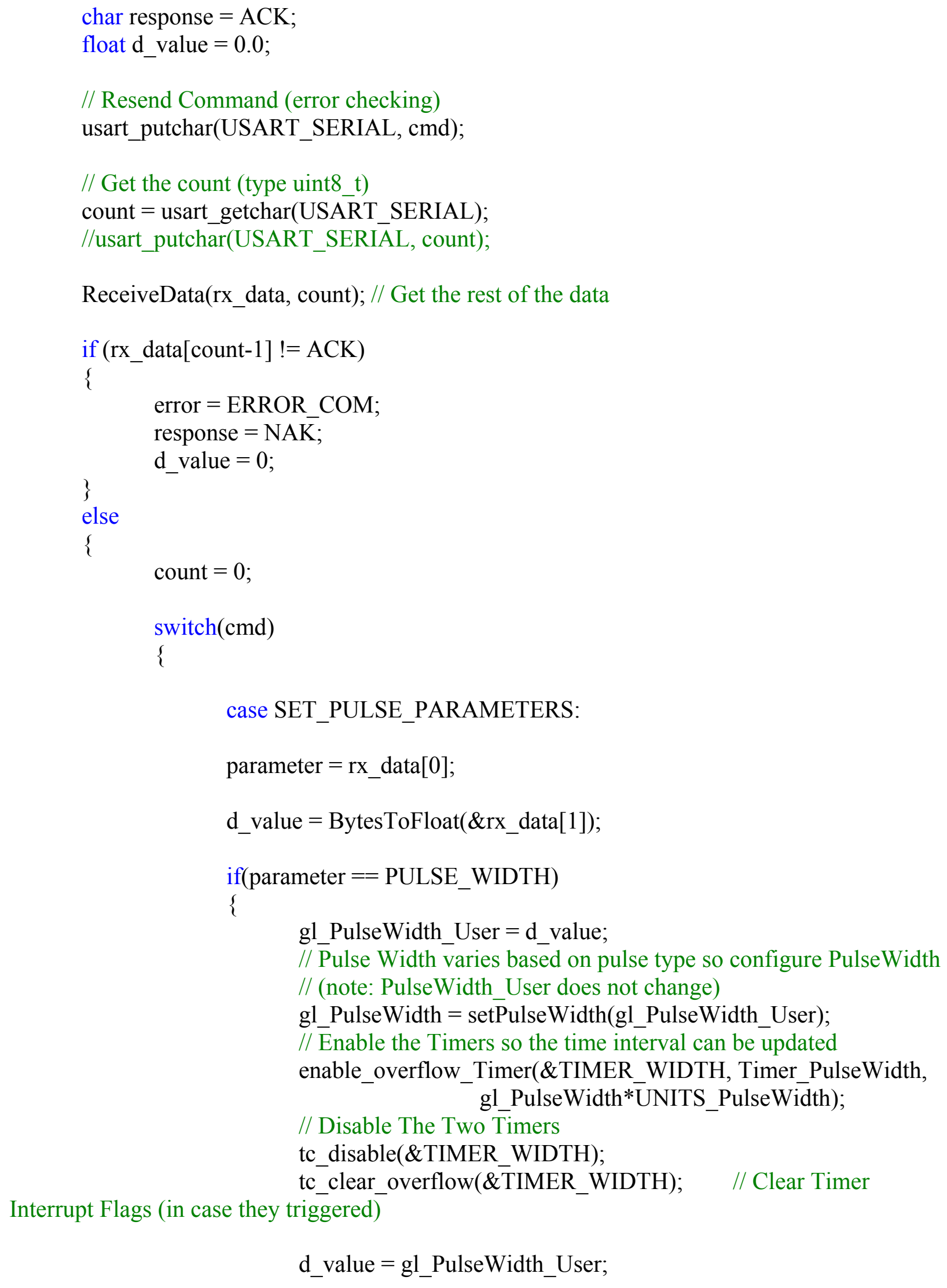




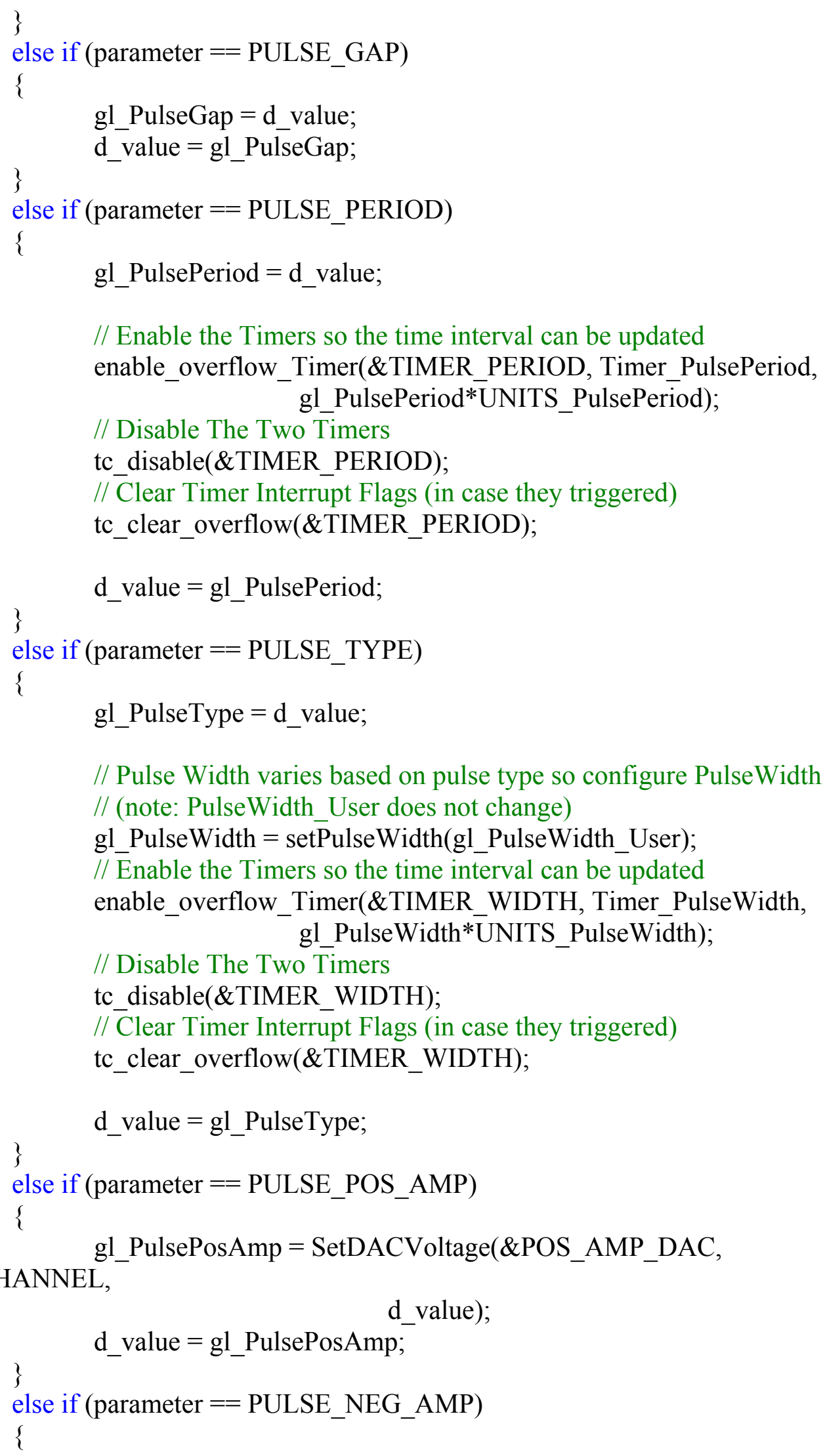


NEG_AMP_DAC_CHANNEL,

$$
\text { gl_PulseNegAmp }=\text { SetDACVoltage }\left(\& N E G \_A M P \_D A C,\right.
$$

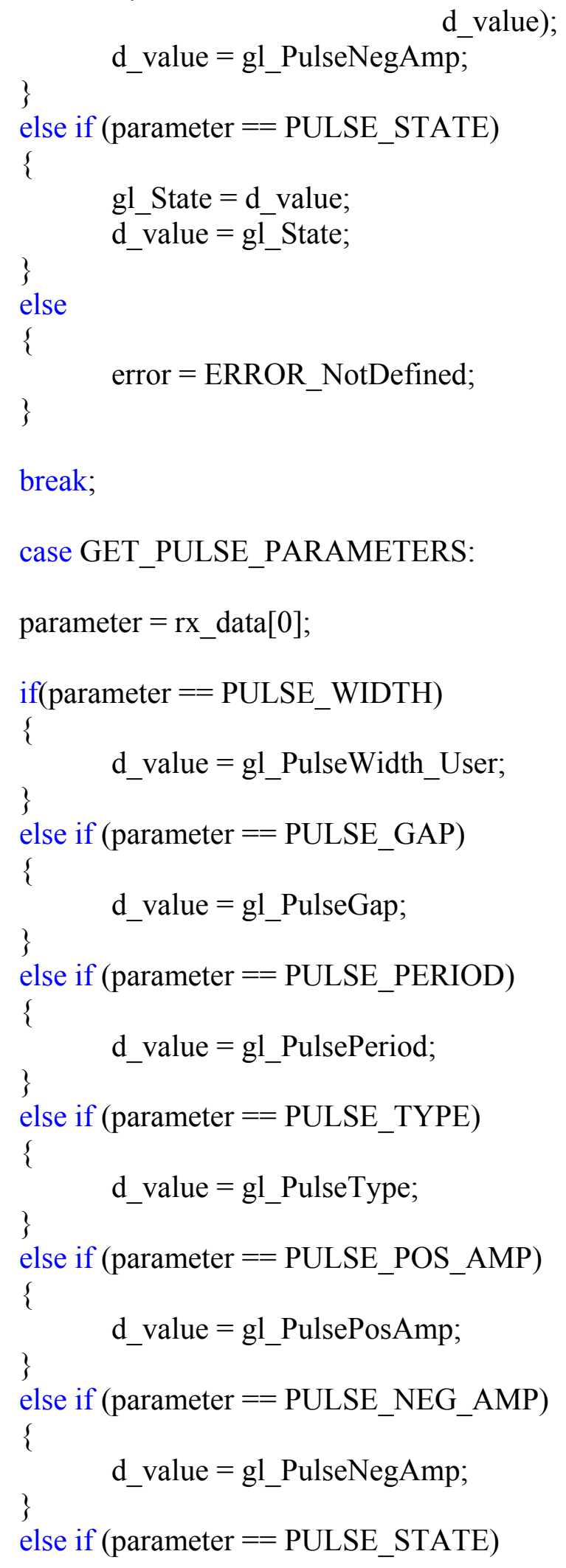




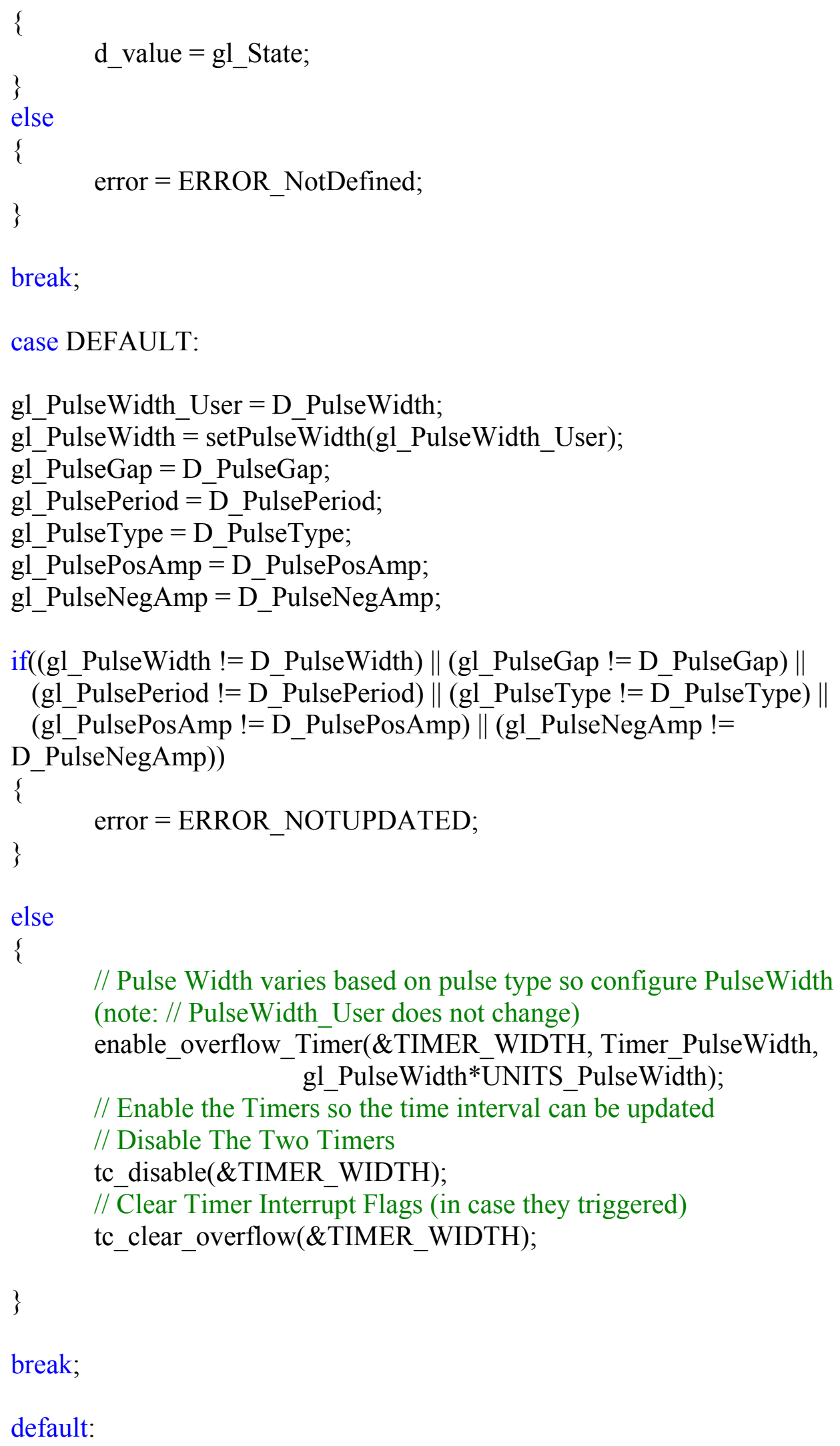




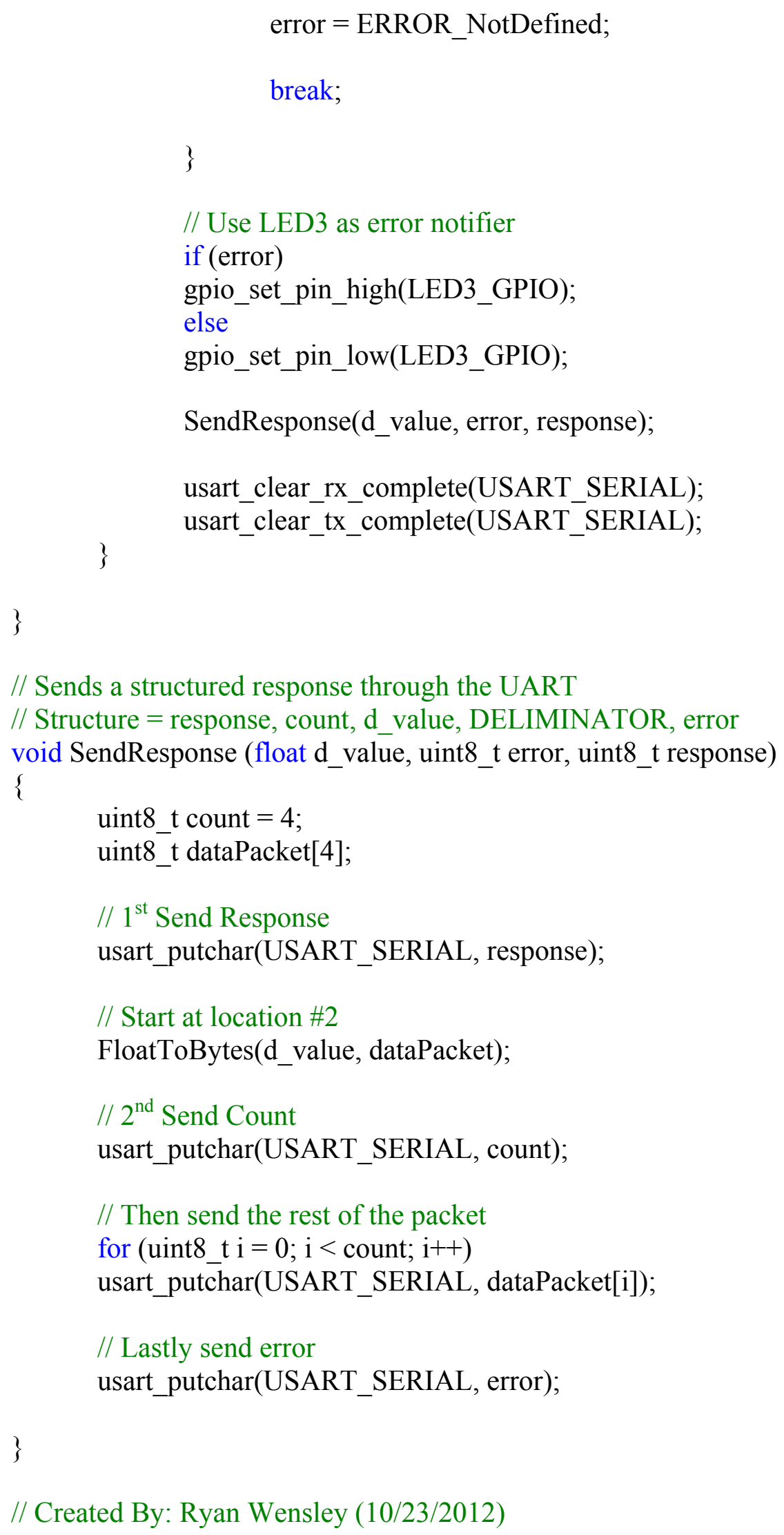


// Sends data from to the TX of the UART of size 'count' void SendData (uint8_t* data, uint8_t count)

\{

static uint8_t $\mathrm{i}=0$;

for $(i=0 ; i<$ count; $i++)$

usart_putchar(USART_SERIAL, *data ++ );

\}

// Created By: Ryan Wensley (10/23/2012)

// Stores data from the RX of the UART of size 'count'

void ReceiveData (uint8_t* data, uint8_t count)

\{

static uint8_t $i=0$;

for $(\mathrm{i}=0 ; \mathrm{i}<$ count; $\mathrm{i}++)$

*data++ = usart_getchar(USART_SERIAL);

\}

// Write a string to the TX of the UART

void WriteString (char* data)

\{

while (*data)

usart_putchar(USART_SERIAL, * data ++ );

\}

D. Timers.c

$/ *$

* File Name: Timers.c

$*$

* Created: 10/23/2012 6:59:01 PM

* Author: wenslr01

$*$ /

\#include "Definitions.h"

void SetupMyTC()

\{

// Setup Counter/Timers 


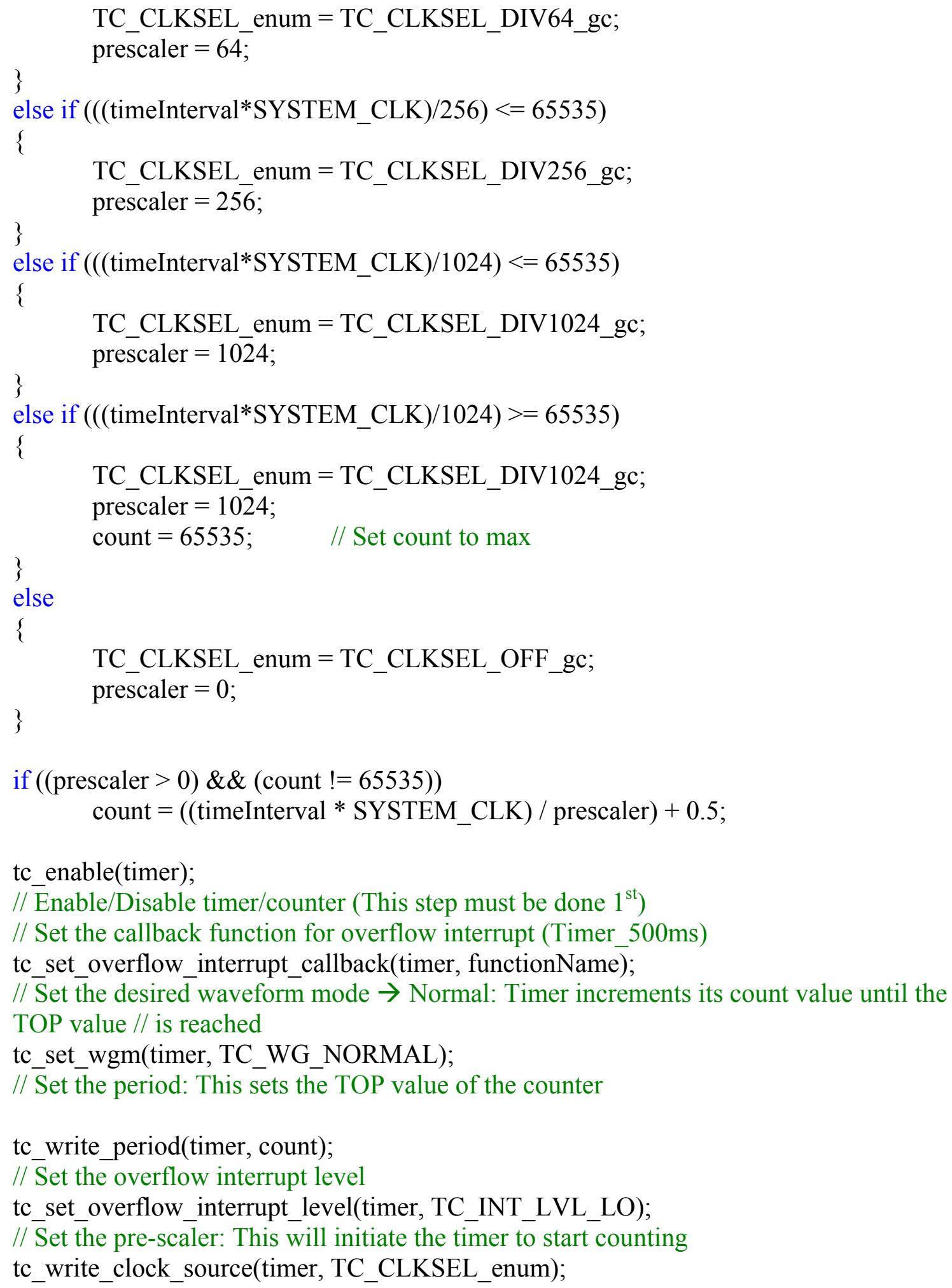


EventQueue_AddEvent(EVENT_PER);

\}

void Timer_PulseWidth(void)

\{

EventQueue_AddEvent(EVENT_PW);

\}

\section{E. Definitions.h}

$/ *$

* File Name: Definitions.h

$*$

* Created: 10/18/2012 10:55:00 AM

* Author: Ryan Wensley

*/

\#include $<$ asf.h $>$

\#include $<$ sysclk.h $>$

\#include $<$ stdio.h $>$

\#include $<$ stdlib.h $>$

\#include $<$ string.h $>$

\#include $<$ math.h $>$

\#ifndef DEFINITIONS_H

\#define DEFINITIONS_H_

// ERROR CODES

\#define LOCATION_UART 0

\#define ERROR_NŌNE 0

\#define ERROR_NotDefined LOCATION_UART+1

\#define ERROR_COM

\#define ERROR_NOTUPDATED

LOCATION_UART+2

LOCĀTION_UART+3

// PORTS
\#define CLKOUT_PORT
\#define IOPORT_PIN
\#define POS_CTRL_PIN
\#define NEG_CTRL_PIN
\#define ON_OFF_PIN
\#define POS_REF_PIN

PORTCFG_CLKOUT_PD7_gc

IOPORT_CREATE_PIN(PORTD, $\overline{7})$

IOPORT_CREATE_PIN(PORTC, 0)

IOPORT_CREATE_PIN(PORTC, 1)

IOPORT_CREATE_PIN(PORTC, 2)

IOPORT_CREATE_PIN(PORTA, 2) 


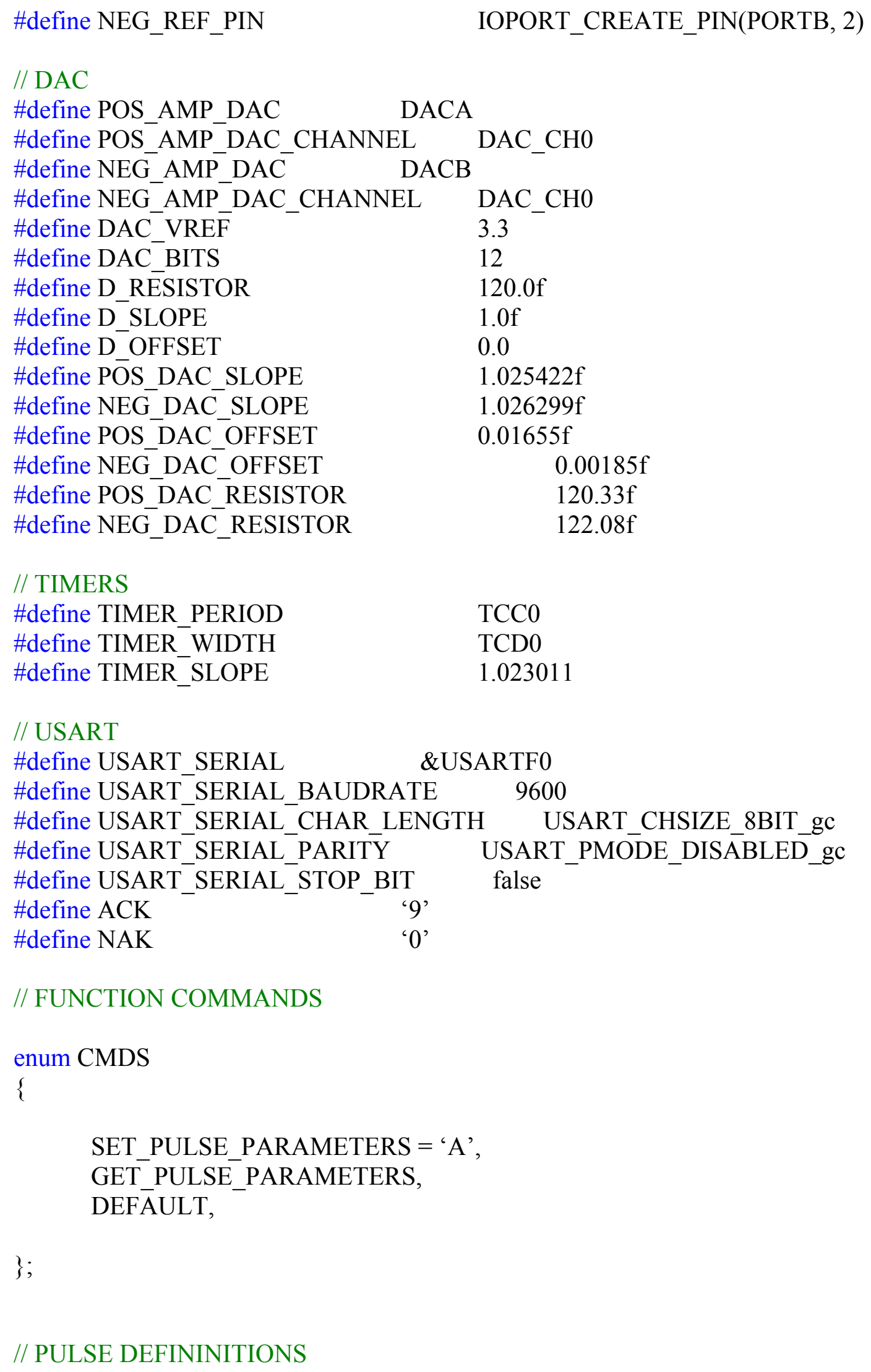


\#define OFF

\#define $\mathrm{ON}$

// PULSE UNITS

\#define UNITS_PulseWidth

\#define UNITS PulsePeriod

\#define UNITS_PulseGap

\#define UNITS_Amplitude

// PULSE DEFAULTS

\#define D_PulseWidth

\#define D_PulseGap

\#define D_PulsePeriod

\#define D_PulseType

\#define D_PulsePosAmp

\#define D_PulseNegAmp

enum PULSES

\{

MONOPOLAR = ' 1 ',

BIPOLAR,

TRIPOLAR,

PENTAPOLAR,

\}

enUm PARAMETERS

\{

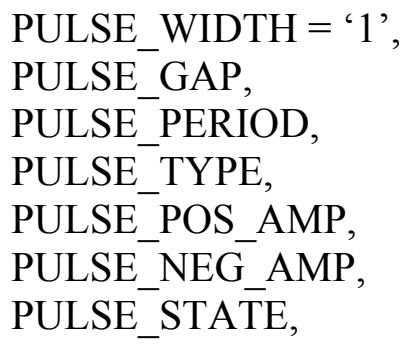

PULSE WIDTH $=$ ' 1 ', PULSE_GAP, PULSE_PERIOD, PULSE_TYPE, PULSE_POS_AMP, PULSE_NEG_AMP, PULSE_STATE,

\}

// States enum STATES

\{

START $=0$,

PHASE1,
0

1

$1 \mathrm{E}-6$

$1 \mathrm{E}-3$

$1 \mathrm{E}-6$

$1 \mathrm{E}-3$

\begin{tabular}{|c|c|}
\hline \multirow{2}{*}{\multicolumn{2}{|c|}{$/ /$ in us }} \\
\hline & \\
\hline 20.0 & $/ /$ in $\mathrm{ms}$ \\
\hline BIPOLAR & // Defined under PULSES \\
\hline 10.0 & $/ /$ in $\mathrm{mA}$ \\
\hline 10.0 & $/ /$ in $\mathrm{mA}$ \\
\hline
\end{tabular}




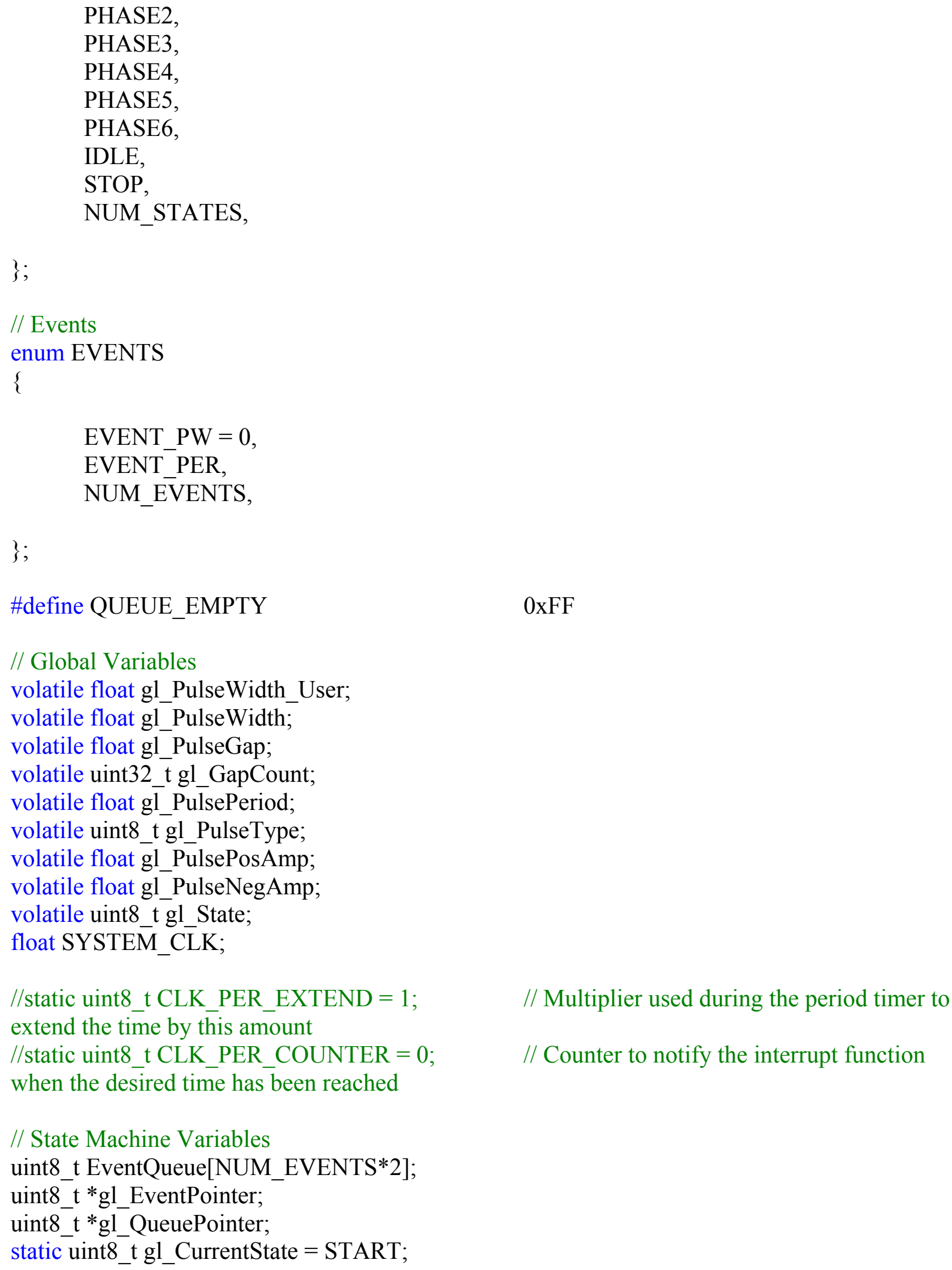




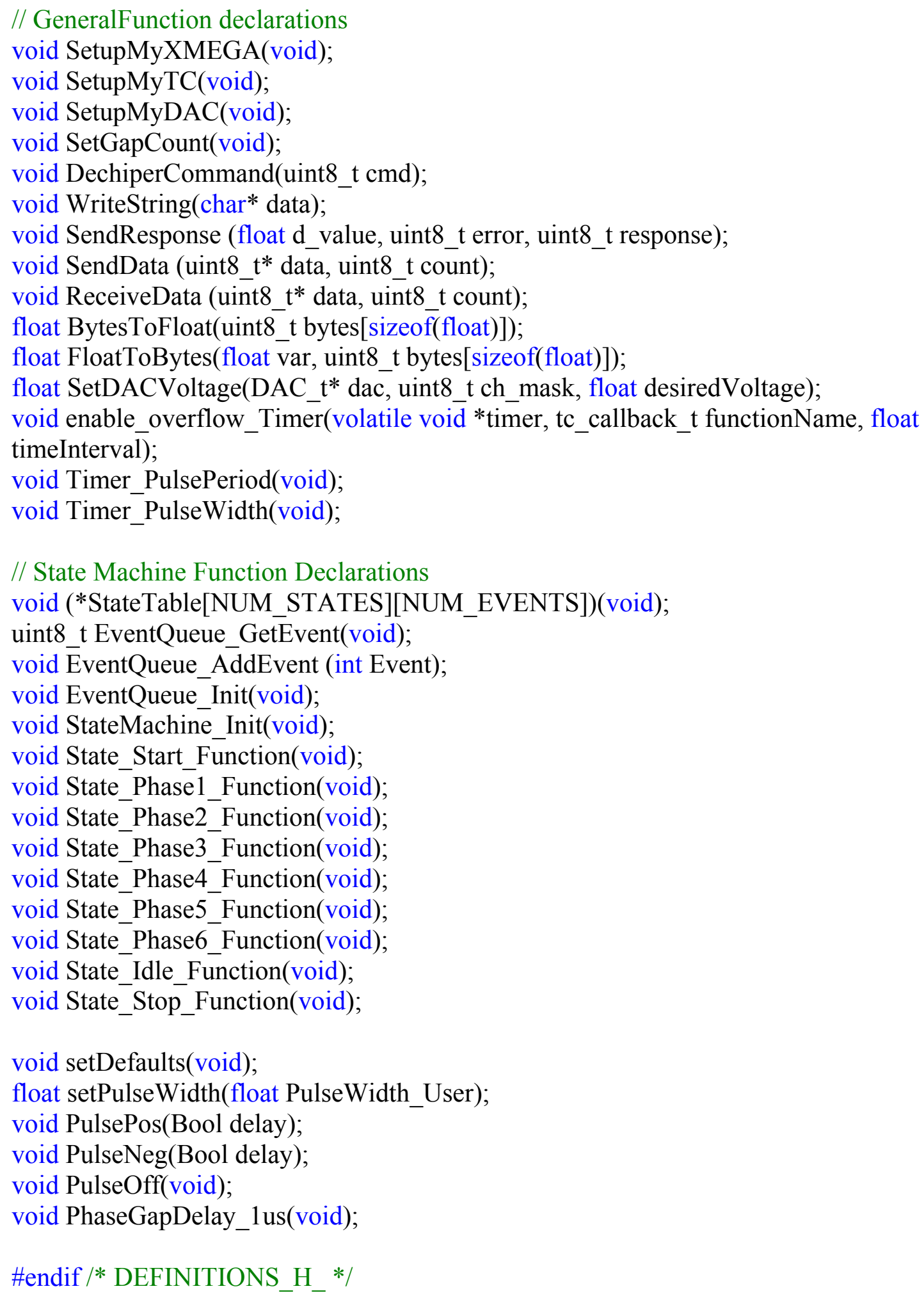




\section{Appendix D: GUI Executable Code}

All code was written in VisualStudios 2010 (C\#). The executable can be run on any computer with the 4.0 Framework installed. Files in the project:

- "Form1.cs"

- "GlobalCache.c"

- "XMEGA.c"

\section{A. Form1.cs}

using System;

using System.Collections.Generic;

using System.ComponentModel;

using System.Data;

using System.Drawing;

using System.Linq;

using System. Text;

using System.Windows.Forms;

using System.IO.Ports;

namespace SCS_PulseGenerator

\{

public partial class Form1 : Form

\{

public Form1()

\{

InitializeComponent();

ComPort.Value $=$ GlobalCache.startPort;

int error $=$ setup_SerialPort();

if $($ error $==0)$

GetAllParams();

\}

private int setup_SerialPort()

\{

int error $=0$;

GlobalCache.XMEGA.Close();

GlobalCache.XMEGA = new SerialPort("COM" + ComPort.Value, Convert.ToInt32(baudRate.Text));

GlobalCache.XMEGA.StopBits = StopBits.One;

GlobalCache.XMEGA.DataBits $=8$; 


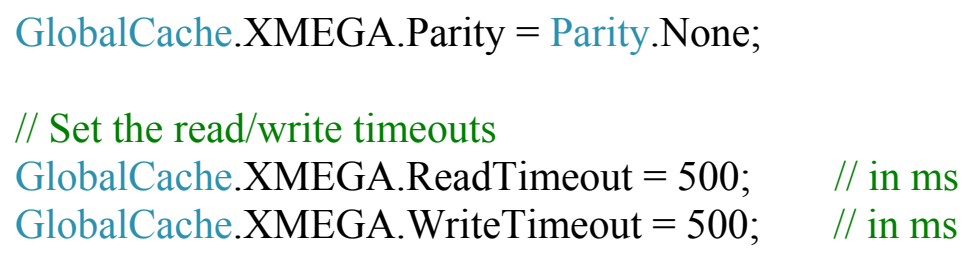


XMEGA.PARAMETERS.PULSE_GAP, ref PG);

error $\mid=$ XMEGA.SendCommand(XMEGA.CMDS.GET_PULSE_PARAMETERS, XMEGA.PARAMETERS.PULSE_PERIŌD, ref PP);

error $\mid=$ XMEGA.SendCommand(XMEGA.CMDS.GET_PULSE_PARAMETERS, XMEGA.PARAMETERS.PULSE_TYPE, ref PT);

error $\mid=$ XMEGA.SendCommand(XMEGA.CMDS.GET_PULSE_PARAMETERS, XMEGA.PARAMETERS.PULSE_POS_AMP, ref PPA);

error $\mid=$ XMEGA.SendCommand(XMEGA.CMDS.GET_PULSE_PARAMETERS, XMEGA.PARAMETERS.PULSE_NEG_AMP, ref PNA);

error $\mid=$ XMEGA.SendCommand(XMEGA.CMDS.GET_PULSE_PARAMETERS, XMEGA.PARAMETERS.PULSE_STATE, $\operatorname{ref}$ ST);

PulseWidth.Value $=$ Convert.ToDecimal(PW);

PulseGap.Value $=$ Convert.ToDecimal $(\mathrm{PG})$;

PulsePeriod.Value $=$ Convert.ToDecimal $(\mathrm{PP})$;

ListPulseType((XMEGA.PULSES)PT);

PulsePosAmp. Value $=$

Convert.ToDecimal(((double)PPA*GlobalCache.POS_DAC_Slope)

+ GlobalCache.POS_DAC_Offset);

PulseNegAmp.Value $=$ Convert.ToDecimal $(($ double $)$ PNA $*$

GlobalCache.NEG_DAC_Slope) +

GlobalCache.NEG_DAC_OOffset);

SetOnOffBtn(ST);

return (error);

\}

private int SetAllParams()

\{

int error $=0$;

float $\mathrm{PW}=($ float $)$ PulseWidth.Value;

float $P G=$ (float)PulseGap.Value;

float $\mathrm{PP}=$ (float)PulsePeriod.Value;

float PT $=$ GetPulseType(PulseType.Text);

float PPA $=($ float $)(($ double $)$ PulsePosAmp.Value $*(1 /$

GlobalCache.POS_DAC_Slope)) - GlobalCache.POS_DAC_Offset);

float PNA $=($ float $)((($ double $)$ PulseNegAmp.Value $*(1 /$

GlobalCache.NEG_DAC_Slope)) - GlobalCache.NEG_DAC_Offset);

float $\mathrm{ST}=$ GetOnOffBtn$($ OnOffBtn.Text, false $)$;

error $\mid=$ XMEGA.SendCommand(XMEGA.CMDS.SET_PULSE_PARAMETERS, XMEGA.PARAMETERS.PULSE_WIDTH, ref PW);

error $\mid=$ XMEGA.SendCommand(XMEGA.CMDS.SET_PULSE_PARAMETERS, XMEGA.PARAMETERS.PULSE_GAP, ref PG);

error $\mid=$ XMEGA.SendCommand(XMEGA.CMDS.SET_PULSE_PARAMETERS,

XMEGA.PARAMETERS.PULSE_PERIOD, ref PP); 


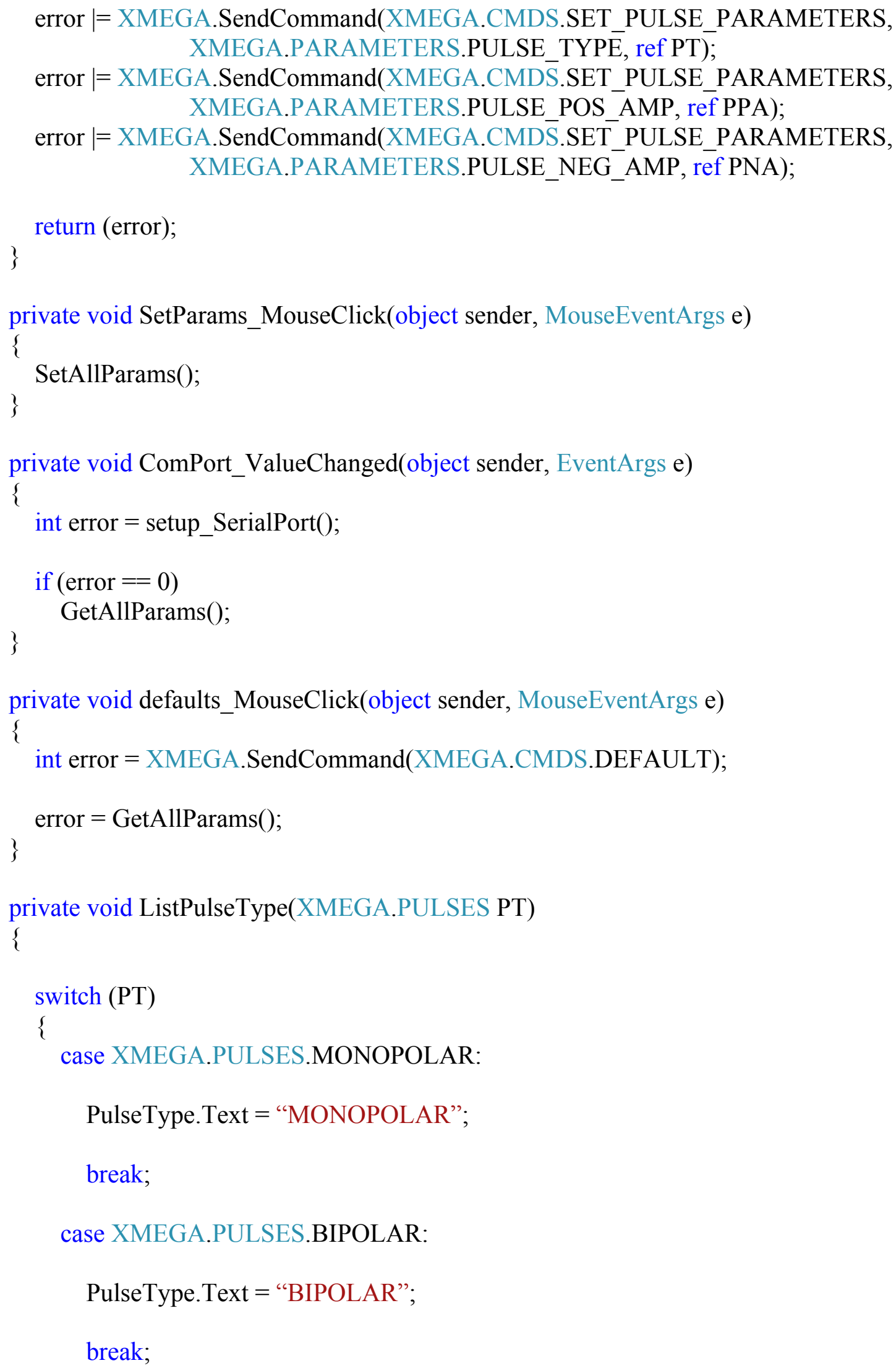




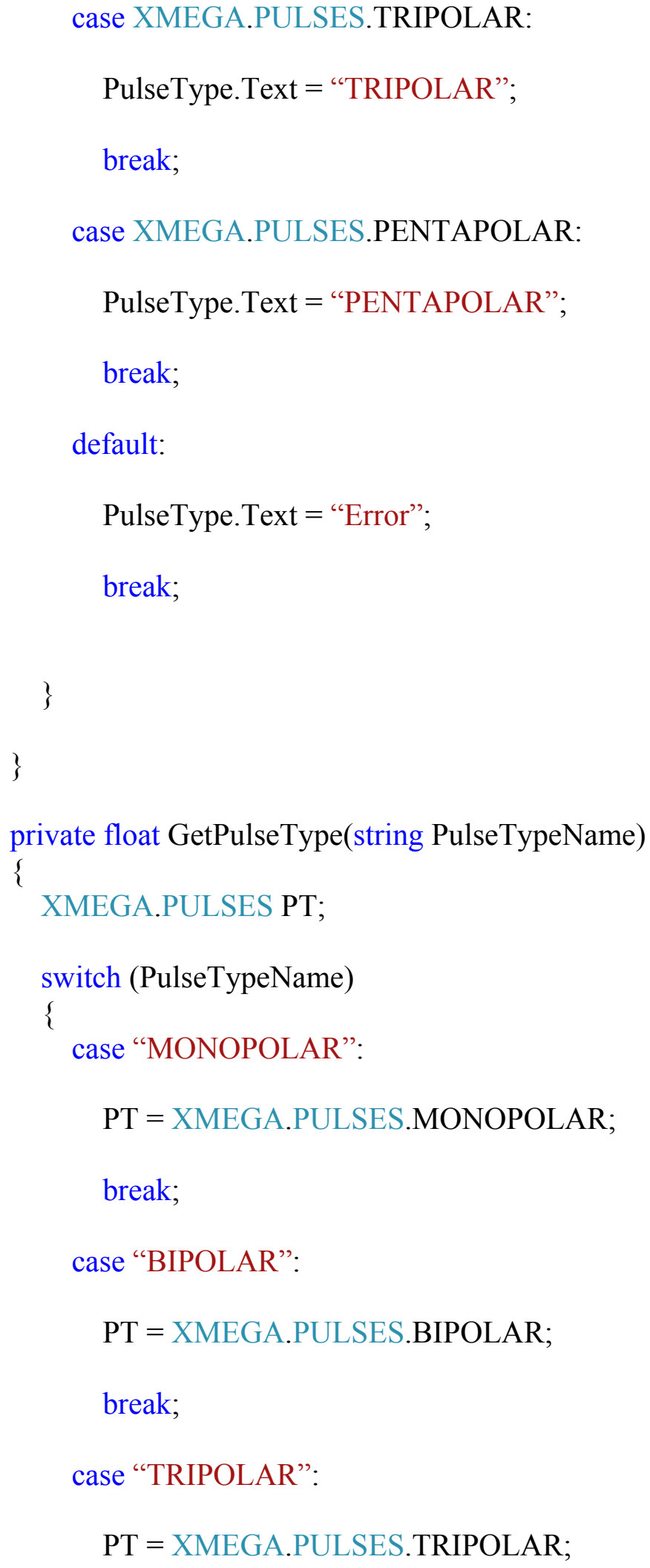




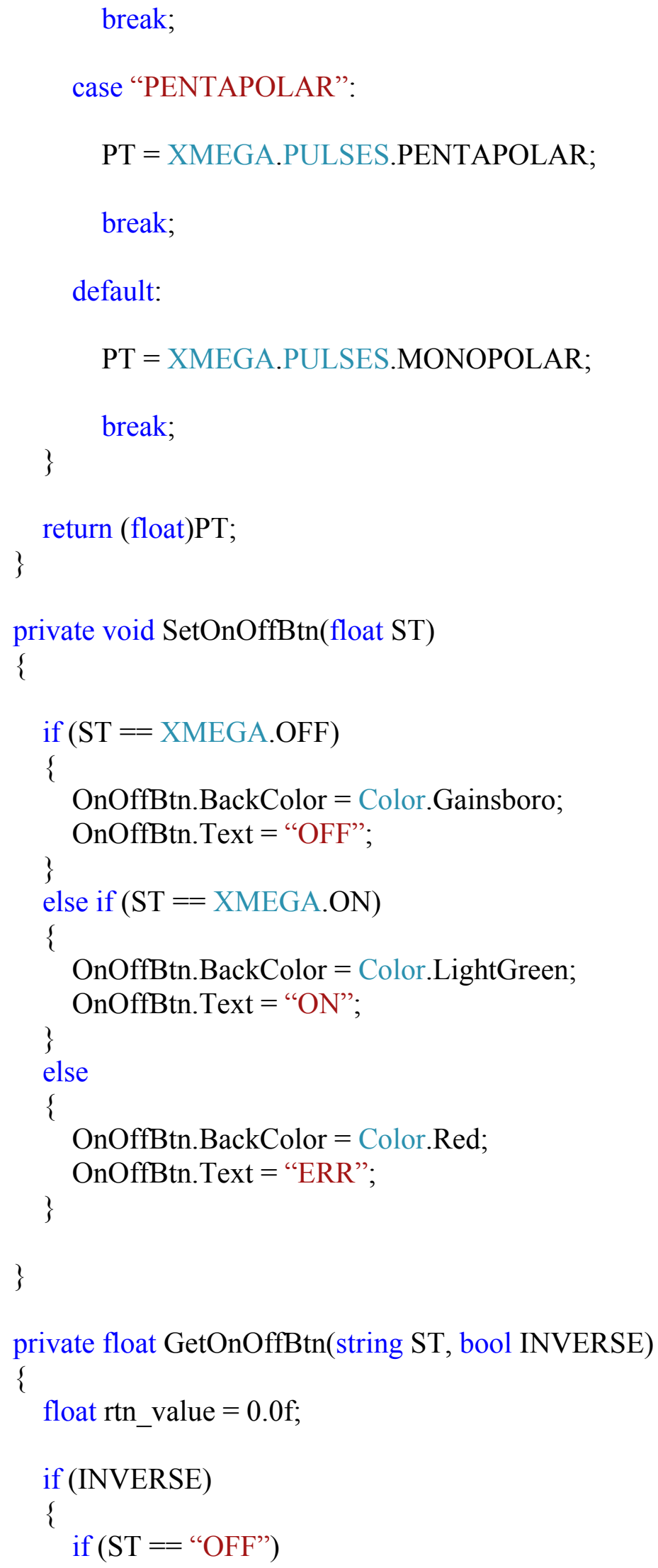




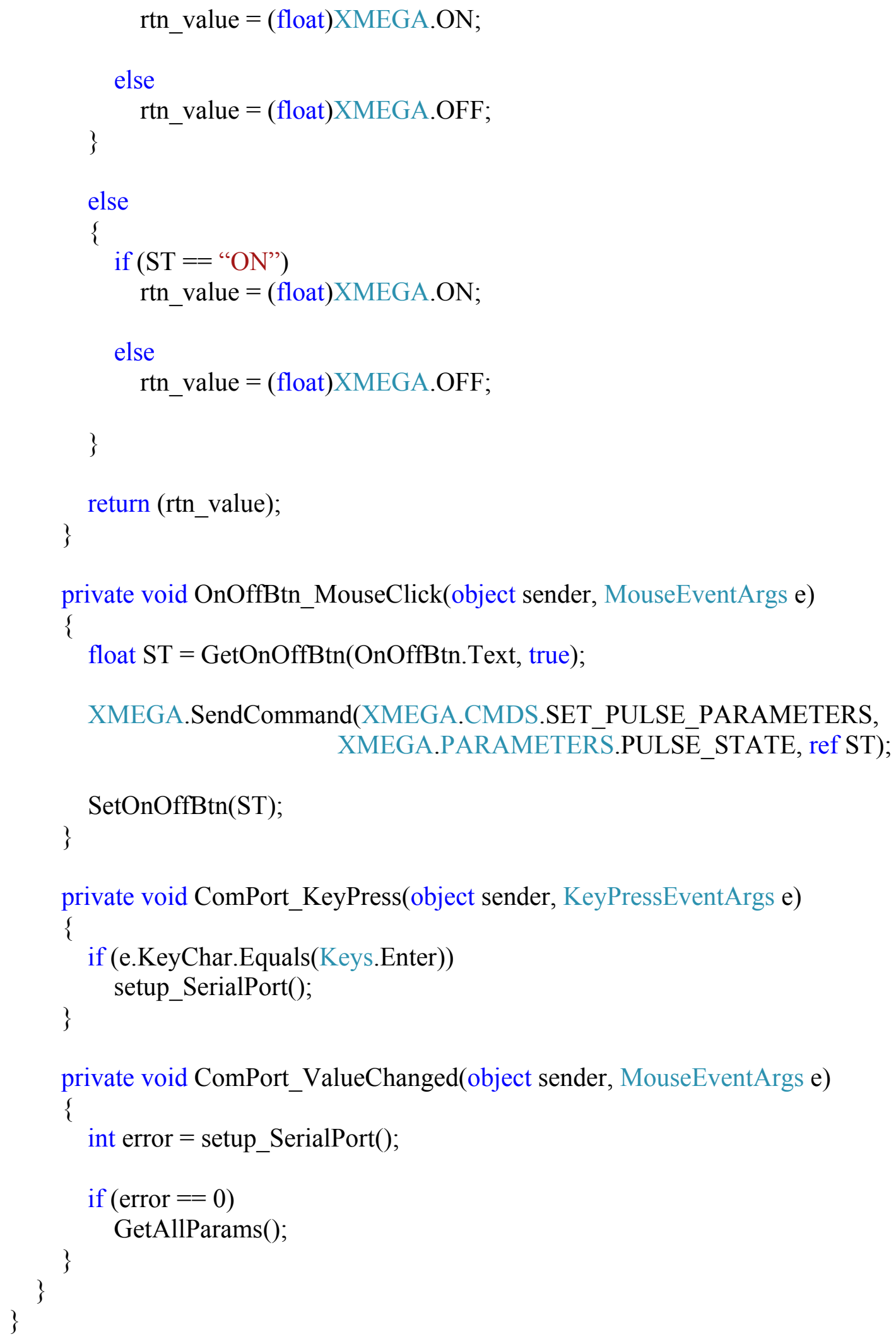




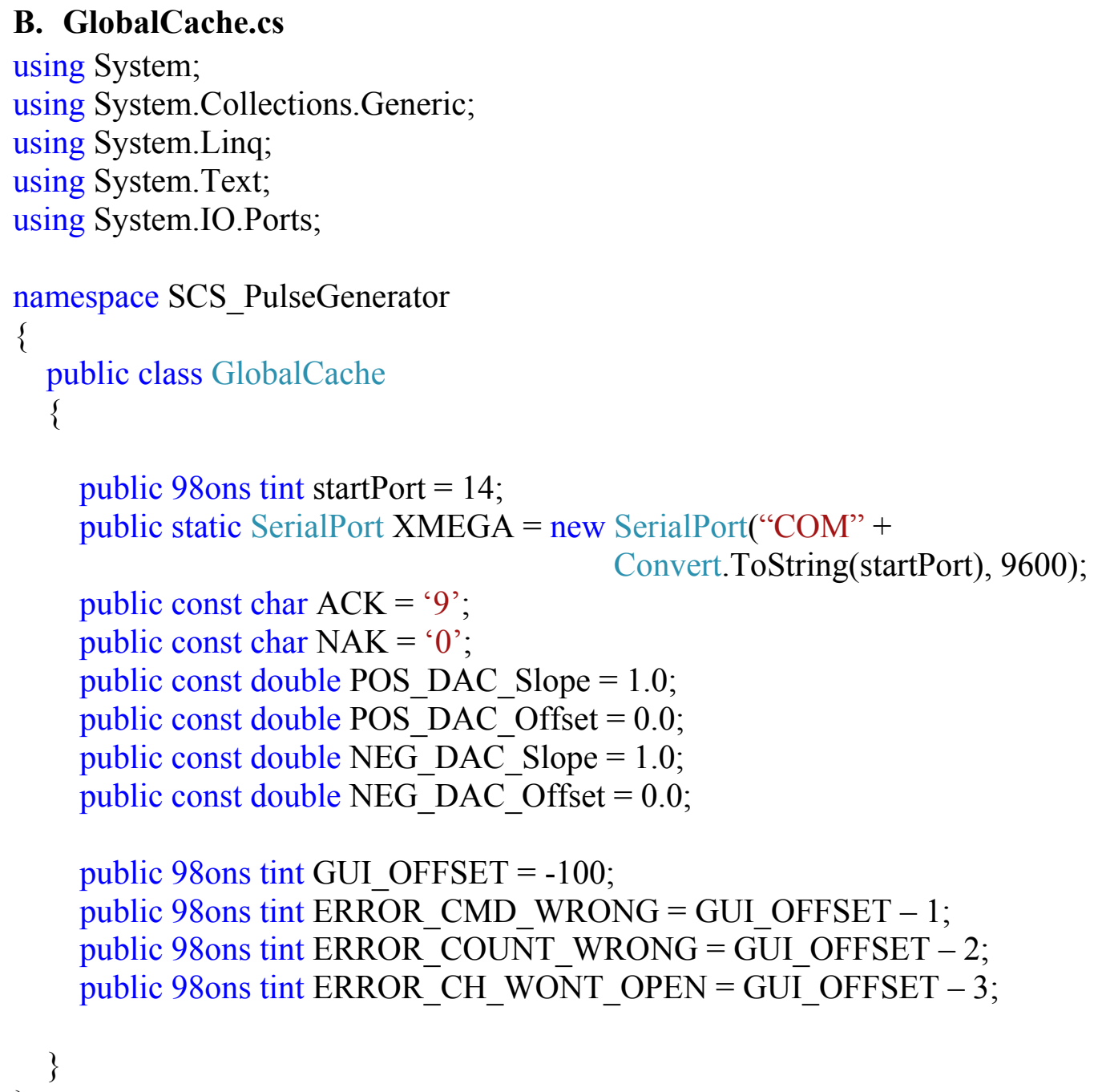

\section{XMEGA.cs}

using System; using System.Collections.Generic; 
public static int SendCommand(CMDS command, PARAMETERS parameter, ref float inputData)

\{

SerialPort XMEGA = GlobalCache.XMEGA;

byte[] tx_data $=$ new byte[6];

byte[] rx_data $=$ new byte[6];

byte[] $\mathrm{cmd}=$ new byte[2];

byte[] count $=$ new byte[1];

byte[] reponse $=$ new byte[1];

byte[] error $=$ new byte[1];

byte[] temp = new byte[1];

XMEGA.DiscardInBuffer();

XMEGA.DiscardOutBuffer();

$/ / 1^{\text {st }}$ Send Command

cmd $[0]=$ Convert.ToByte(command);

XMEGA.Write(cmd, 0, 1); // Send CMD

XMEGA.Read(cmd, 1, 1); // Get CMD Back

if $(\mathrm{cmd}[0] !=\mathrm{cmd}[1])$

\{

XMEGA.DiscardInBuffer();

XMEGA.DiscardOutBuffer();

\}

return GlobalCache.ERROR_CMD_WRONG;

$/ / 1^{\text {st }}$ Byte of packet $=$ Parameter

tx_data $[0]=$ Convert.ToByte(parameter);

count $[0]++$;

if $(\mathrm{cmd}[0]==$ Convert.ToByte(CMDS.SET_PULSE_PARAMETERS $))$

\{

// 2-5 Bytes of packet $=$ float value (converted to bytes)

float pre_value $=$ inputData;

byte[] d_value = BitConverter.GetBytes(pre_value);

pre_value $=$ BitConverter.ToSingle $\left(d_{-}\right.$value, 0$)$;

for (int $\mathrm{i}=1 ; \mathrm{i}<=4 ; \mathrm{i}++$ )

tx_data[i] =d_value $[\mathrm{i}-1]$;

count $[0]+=4$

\}

$/ / 6^{\text {th }}$ Byte of packet $=$ response 


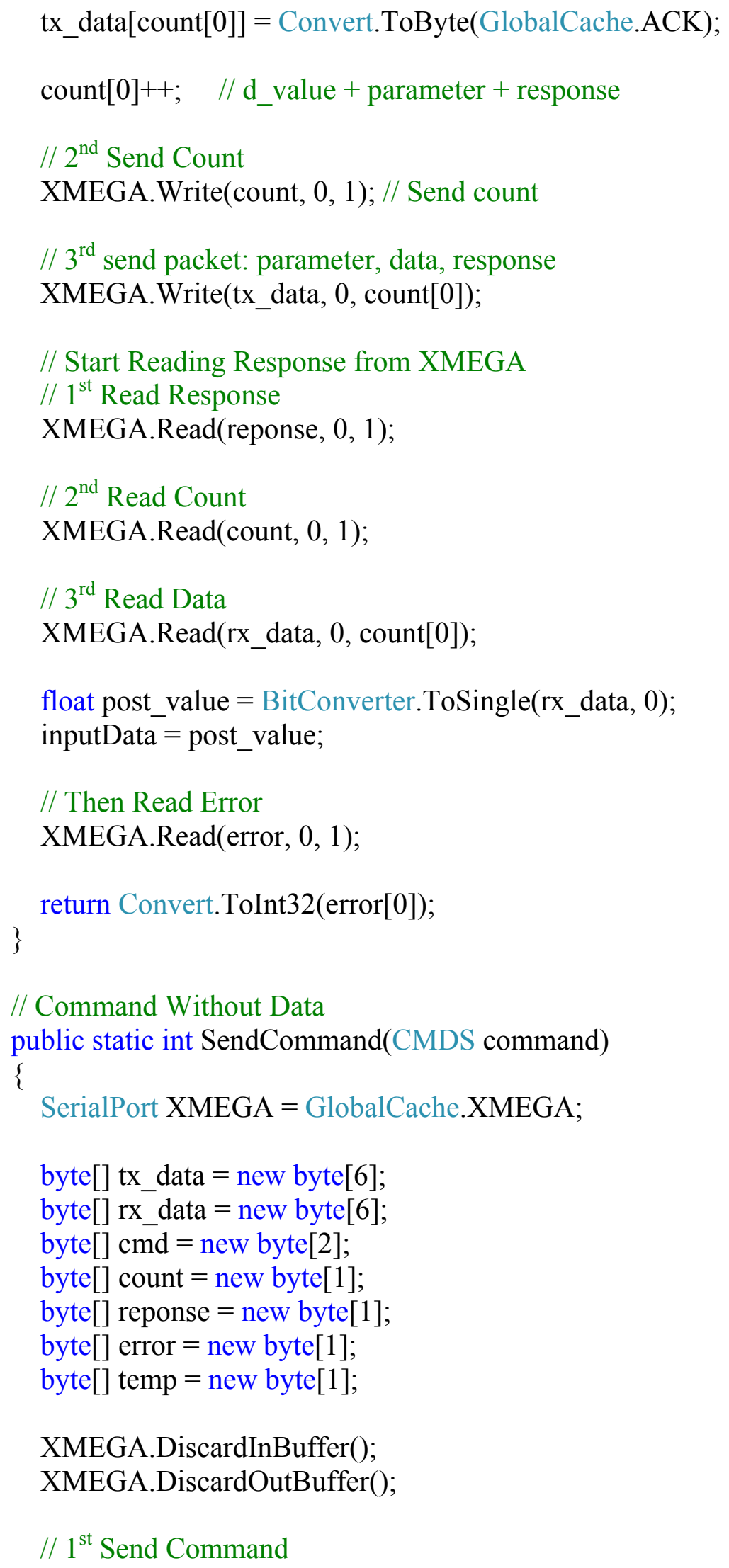




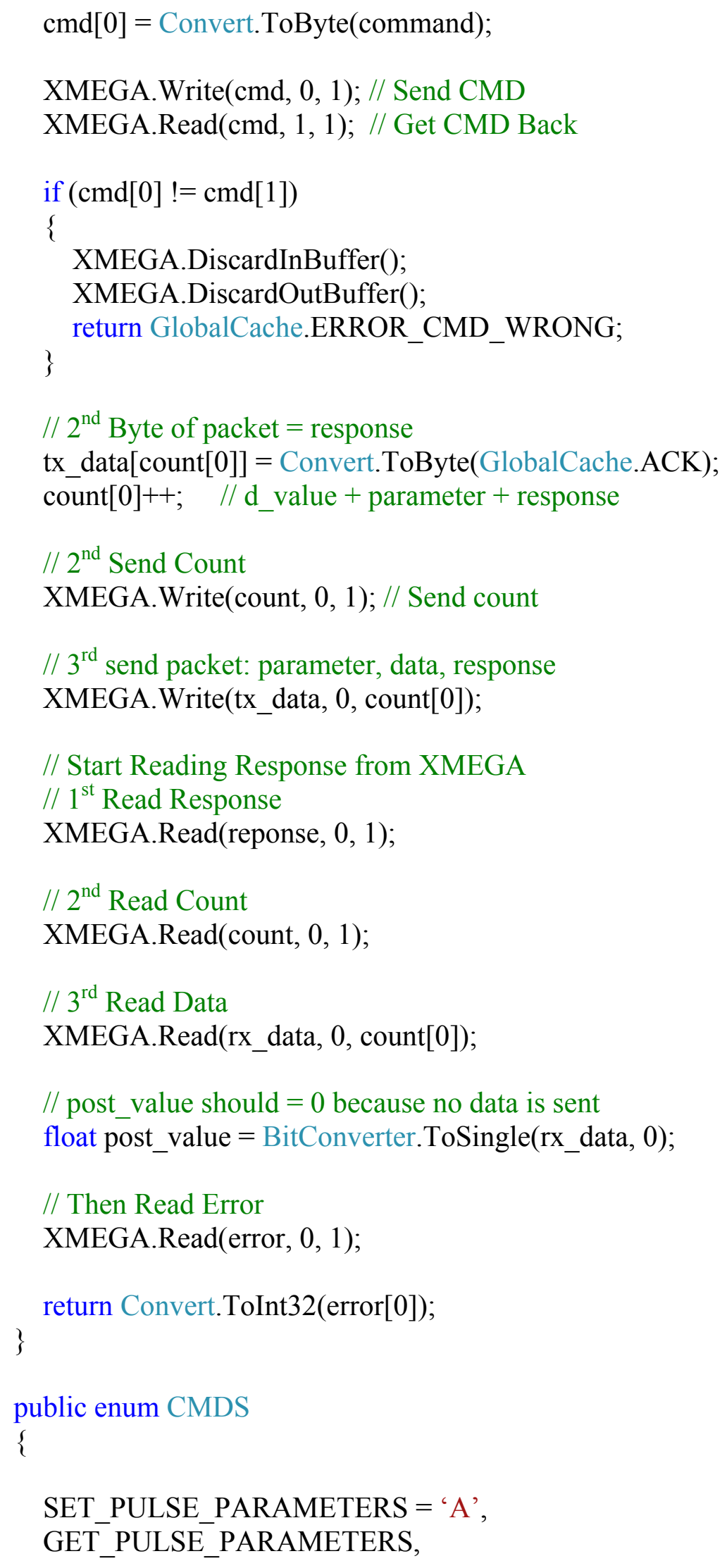




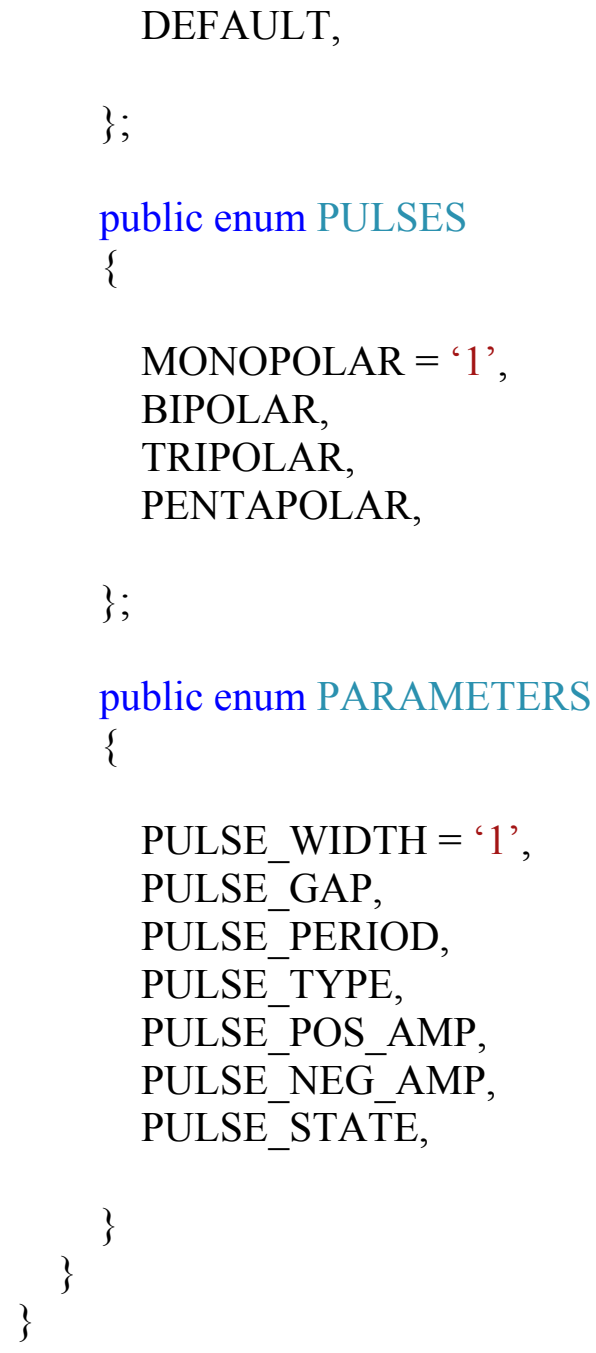




\section{Appendix E: Hardware Schematics}

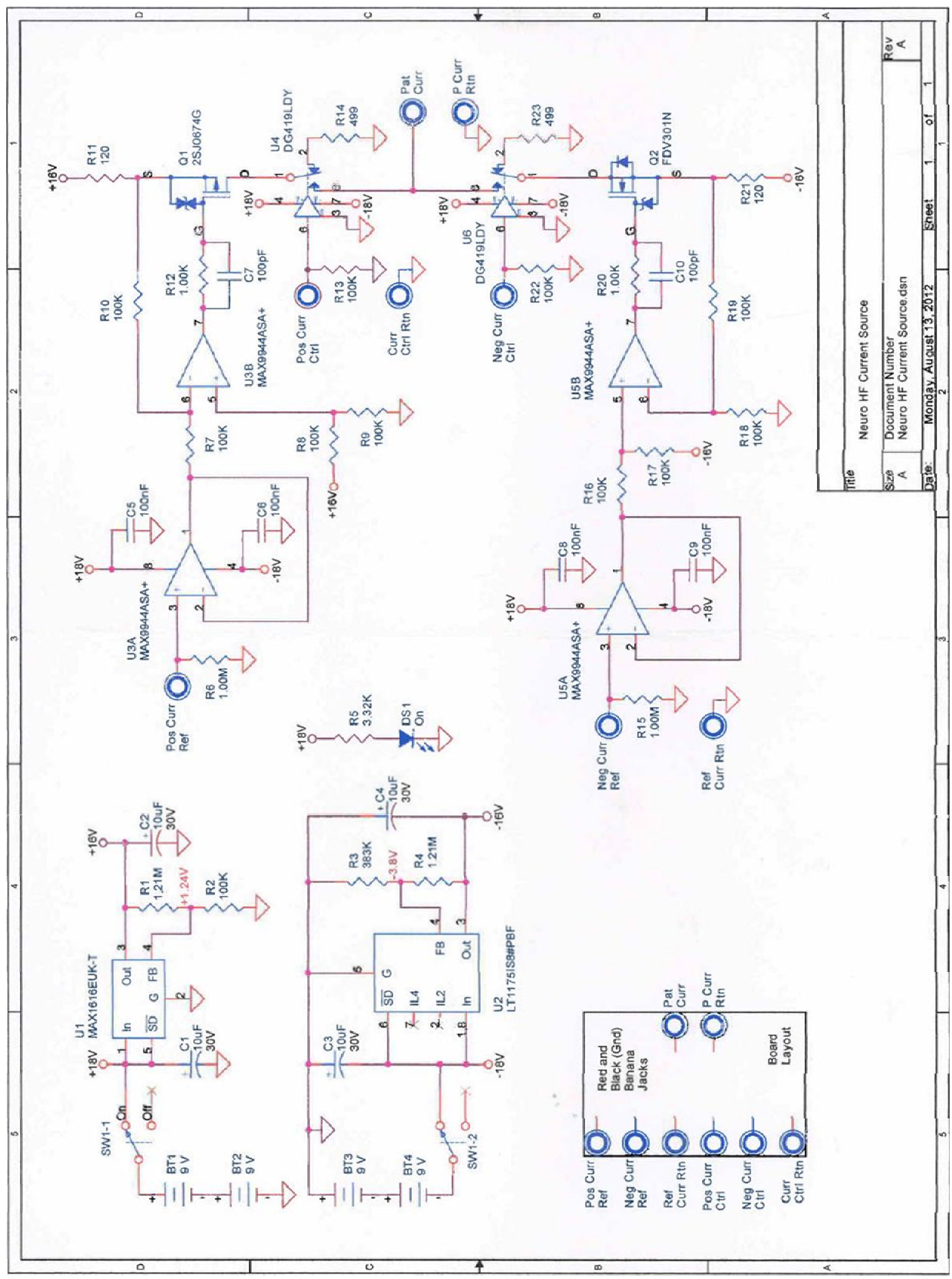

Figure 42: V-I Pulse Converter 


\section{Appendix F: Equipment}

Table 7: Equipment List for Saline Experiment

\begin{tabular}{|l|l|l|}
\hline Manufacturer & Model & Description \\
\hline Tektronix & TDS3014C & $\begin{array}{l}\text { Digital Recording } \\
\text { Oscilloscope }\end{array}$ \\
\hline Tektronix & ADA400A & $\begin{array}{l}\text { Differential Amplifier Scope } \\
\text { probe }\end{array}$ \\
\hline Keithley & 2400 & Source Measure Unit (SMU) \\
\hline Metrohm & 712 Conductometer & $\begin{array}{l}\text { High accuracy conductance } \\
\text { meter }\end{array}$ \\
\hline Thermo Scientific & Barnstead Nanopure & Deionized water \\
\hline Intell-Lab & - & Balance scale \\
\hline St. Jude Medical & $7131 / 60$ & IS-1 BI ICD Lead \\
\hline St. Jude Medical & - & Octal SCS Lead \\
\hline
\end{tabular}

